\title{
A Refined Vs30 Map for Taiwan Based on Ground Motion Attenuation Relationships
}

\author{
Kun-Sung Liu ${ }^{1, *}$ and Yi-Ben Tsai ${ }^{2}$ \\ ${ }^{1}$ Department of Civil Engineering \& Hazard Mitigation Research Center, Kao Yuan University, Kaohsiung, Taiwan, R.O.C. \\ ${ }^{2}$ Pacific Earthquake Science Associates, Los Altos, California, USA
}

Received 21 January 2013, revised 9 January 2015, accepted 11 May 2015

\begin{abstract}
Seismic hazard evaluations require an estimate of the expected ground motion at the site of interest usually by using attenuation relationships. The mean shear-wave velocity over the top $30 \mathrm{~m}$ (Vs30) is incorporated in the ground motion attenuation relationships in this study. By comparing the standard deviations of the residuals between the observed and predicted values before and after incorporating the site effect term Vs30, the reduction in standard deviation for the peak ground velocity (PGV) is significantly reduced by about $11 \%$. Clearly, the refined attenuation relationships will be more useful for engineering purposes. Analyzing the site effect term using the amplification factor (relative to a site with Vs30 $=760 \mathrm{~m} \mathrm{~s}^{-1}$ ), has revealed that the Changhua Plain, Chianan Plain, Pingtung Valley, Ilan Plain, and Taipei Basin have high values, implying large ground motion amplification. Following a disastrous earthquake, quick assessment and timely peak ground acceleration (PGA) and PGV map reporting will be critical for effective emergency response operations. After an earthquake we can combine the simple attenuation relationships, as determined from Model 1, to provide near real-time estimation and reporting of the PGA and PGV values for the Taiwan area. We can also use the relations between the intra-event site residual and the Vs30 to estimate the Vs30 for stations that have recorded strong motions, but do not yet have Vs30 information. Our approach including sites with estimated Vs30 has resulted in a refined Vs30 contour map that can be used for more realistic seismic hazard assessment for Taiwan. This approach is especially applicable to the foothill and mountain areas.
\end{abstract}

Key words: Attenuation relationship, Peak Ground Acceleration (PGA), Peak Ground Velocity (PGV), Taiwan, Vs30

Citation: Liu, K. S. and Y. B. Tsai, 2015: A refined Vs30 map for Taiwan based on ground motion attenuation relationships. Terr. Atmos. Ocean. Sci., 26, 631-653, doi: 10.3319/TAO.2015.05.11.01(TC)

\section{INTRODUCTION}

Local site effects play an important role in modifying seismic motions. This fact is evidenced by the observed damage distribution patterns in numerous earthquakes and is reflected in some seismic codes and ground-motion prediction equations (GMPEs), which prescribe stronger motions for sites located in soft sediments compared to bedrock sites (Borcherdt 1970). Local site conditions at an accelerograph station can dramatically affect the strong motions recorded (Douglas 2003). In particular, soft sediments with low seismic velocities relative to the underlying bedrock can lead to large ground motion amplification at the surface (Pratt et al. 2003). Some studies have found that shallow soil sites have significantly higher ground motions than rock or stiff soil

\footnotetext{
* Corresponding author

E-mail:tf0143@cc.kyu.edu.tw
}

sites (Campbell 1981). Site-effects estimation has been a major issue in engineering seismology for the last 20 years. This is due to the fact that seismic hazard is strongly influenced by site effects because site conditions strongly affect the frequency content and also the amplitude of ground motions. This is also because most populated areas are located in sedimentary basins (Drouet et al. 2008).

The average shear-wave velocity of the upper $30 \mathrm{~m}$ of a soil profile (Vs30) is used as a quantitative parameter for most earthquake ground-motion site-effect studies (Boore et al. 1993; Anderson et al. 1996; Bolt and Abrahamson 2003; Huang et al. 2007, 2009; Lee and Tsai 2008). The Vs30 is adopted as an international standard for soil classification since it was proposed by the United States National Earthquake Hazard Reduction Program (NEHRP) (Gallipoli and Mucciarelli 2009). Starting from the work of Borcherdt (1994), 
the Vs30 has become a standard parameter for quantifying response at a site. Accordingly, successive NEHRP regulations (NEHRP 1994, 1997, 2001) included Vs30 to define their respective local site categories (Cadet et al. 2010). For the development of ground-motion models, developers systematically evaluated a list of predictor parameters to consider for predicting earthquake shaking intensity. The most significant decision made by all developers was to use Vs30 as a parameter for characterizing soil-stiffness effects on ground motions (Power et al. 2008). For example, the "Next Generation Attenuation of Ground Motions Project (NGA)" of the Pacific Earthquake Engineering Research Center (PEER) has directly used Vs30 for the ground-motion attenuation models (Power et al. 2008). In Taiwan, Huang et al. $(2007,2009)$ used the Vs30 data in Central Taiwan and in the Taipei Basin to estimate the high frequency site amplification using the quarter-wavelength method. Lee et al. (2012) developed a new empirical Arias intensity attenuation relationship for shallow crustal earthquakes in Taiwan considering Vs30. Their results show that the incorporation of Vs30 can significantly reduce regression error.

Several studies have been made to evaluate the site effects based on accelerographic recordings. For example, examination of the residuals for sites with different soil categories was shown to be a useful method using sets of records where site information was not complete (Abrahamson and Litehiser 1989). Liu and Tsai (2005) analyzed the residuals of peak ground acceleration (PGA) and peak ground velocity (PGV) with respect to site conditions. They found the contour maps of site residuals for the PGA and PGV data, especially for the PGV, were highly correlated with the regional geology and topography of Taiwan. They further pointed out that almost all major metropolitan areas coincided with high residual areas that would require special attention in structural seismic design. Choi and Stewart (2005) evaluated the ground motion amplification factors from residuals between accelerations from recordings and attenuation relationships for active seismic regions. They also found site amplification to increase with decreasing Vs30.

In this study we first develop refined attenuation relationships by incorporating a site effect term related to Vs30, the average shear-wave velocity in the upper $30 \mathrm{~m}$ of sediments, aiming to reduce the standard deviation of the predicted ground motion in large earthquakes. We will also investigate the variation in residuals with Vs30 in order to construct a refined Vs30 map for the entire Taiwan area. The results of this study will provide valuable information for site evaluation of critical facilities in high earthquake hazard regions, as well as for land-use planning.

\section{DATA}

Beginning in 1991 the Central Weather Bureau Seismology Center (CWBSC) embarked on a six-year seismic strong-motion instrumentation program, known as the Taiwan Strong Motion Instrumentation Program (TSMIP) (Liu et al. 1999). The TSMIP accelerographic network dataset used in this study consists of 617 free-field stations, primarily covering densely populated areas. The locations of these stations are given in Table 1 and shown in Fig. 1. These free-field stations are densely spaced approximately $5 \mathrm{~km}$ apart, and only about $3 \mathrm{~km}$ apart in urban areas. Concurrently, the Institute of Earth Science, Academia Sinica installed another strong motion network with similar instrumentation in the Central Mountain Strong Motion Array (CMSMA) to provide strong motion data for the mountainous areas (http://www.earth.sinica.edu.tw/ smdmc/cma/cma.htm). The locations of these 10 stations are given in Table 2 and shown in Fig. 1. Strong seismic ground motion data obtained from a total of 627 stations from the TSMIP and CMSMA networks are used in this study to derive new ground motion attenuation relationships.

The Central Weather Bureau (CWB) and National Center for Research on Earthquake Engineering (NCREE) have undertaken a free-field strong-motion station drilling project to construct an engineering geological database for TSMIP (EGDT) since 2000. A total of 468 free-field strong motion stations were surveyed by 2010 , with 439 of these stations drilled and their $\mathrm{P}$ - and $\mathrm{S}$-wave velocities measured using a suspension PS-logging system (Kuo et al. 2011, 2012). Measured Vs30 records are available at 426 of the 617 TSMIP free-field strong-motion stations. Records from these stations are used to develop the attenuation relationships which incorporate a site effect term, aimed to reduce the standard deviation of the predicted ground motion for engineering applications. The crosses in Fig. 1 represent the TSMIP stations with available Vs30 records. Relevant localities are also indicated.

Over 7900 digital accelerograms were recorded from 51 crustal earthquakes by the TSMIP and CMSMA networks, as shown in Fig. 1, and selected for this study (Liu and Tsai 2005). The $\mathrm{M}_{\mathrm{w}}$ magnitudes range from 4.0 - 7.1. These records are analyzed to investigate the attenuation relationship dependence on magnitude, regional earthquake clusters and site effects. Figure 2 shows the data distribution of these events in terms of magnitude and source-to-site distance.

\section{METHODOLOGY}

The strong-motion attenuation relationships express earthquake ground motion parameters as functions of simple parameters characterizing the earthquake source, the propagation path between the earthquake source and the site, and the geological conditions beneath the site. The following equation form is used in this study (Liu and Tsai 2005):

Model 1: The equation includes only magnitude and distance terms. 
$\ln (\mathrm{PGA}, \mathrm{PGV})=\mathrm{a} \ln \left[\mathrm{X}+\mathrm{h}_{1} \exp ^{\left(\mathrm{h}_{2} \mathrm{M}_{\mathrm{w}}\right)}\right]+\mathrm{bX}+\mathrm{cM}_{\mathrm{w}}+\mathrm{d}$

Model 2: The equation includes an additional site term (Vs30).

$$
\begin{array}{r}
\ln (\mathrm{PGA}, \mathrm{PGV})=\mathrm{a} \ln \left[\mathrm{X}+\mathrm{h}_{1} \exp ^{\left(\mathrm{h}_{2} \mathrm{M}_{\mathrm{w}}\right)}\right]+\mathrm{bX}+ \\
\mathrm{cM}_{\mathrm{w}}+\mathrm{d}+\mathrm{e} \ln (\mathrm{Vs} 30 / \mathrm{Vref})
\end{array}
$$

or

$$
\begin{array}{r}
\ln (\mathrm{PGA}, \mathrm{PGV})= \\
\operatorname{aln}\left[\mathrm{X}+\mathrm{h}_{1} \exp ^{\left(\mathrm{h}_{2} \mathrm{M}_{\mathrm{w}}\right)}\right]+\mathrm{bX}+ \\
\mathrm{cM}_{\mathrm{w}}+\mathrm{d}^{\prime}+\mathrm{e} \ln (\mathrm{Vs} 30 / 760)
\end{array}
$$

where PGA and PGV are ground motion parameters, $\mathrm{X}$ is the closest distance to the rupture surface or hypo central distance. We characterize the source-to-site distance in terms of the closest distance to the rupture surface, $r_{\text {rup}}$. If the rupture surface is not defined for an event, the hypo central distance

\begin{tabular}{|c|c|c|c|c|c|c|c|c|c|c|c|c|c|c|c|c|}
\hline \multirow{3}{*}{$\begin{array}{r}\text { No. } \\
1\end{array}$} & \multirow{3}{*}{$\begin{array}{l}\text { Stname } \\
\text { TAP001 }\end{array}$} & \multirow{3}{*}{$\begin{array}{c}\begin{array}{c}\text { Lat } \\
\left({ }^{\circ} \mathbf{N}\right)\end{array} \\
25.04\end{array}$} & \multirow{3}{*}{$\begin{array}{c}\begin{array}{c}\text { Long } \\
\left({ }^{\circ} \mathbf{E}\right)\end{array} \\
121.51\end{array}$} & \multicolumn{4}{|c|}{ TotalRes } & \multicolumn{4}{|c|}{ IntraRes } & \multirow{3}{*}{\begin{tabular}{|l|}
$\mathbf{R}$ \\
5
\end{tabular}} & \multirow{3}{*}{$\frac{\text { Site } \dagger}{E}$} & \multirow{3}{*}{$\begin{array}{c}\begin{array}{c}\text { Vs30 } \\
\left(\mathrm{m} \mathrm{s}^{-1}\right)\end{array} \\
160.1\end{array}$} & \multirow{3}{*}{\begin{tabular}{|c|} 
Site \\
$E$
\end{tabular}} & \multirow{3}{*}{\begin{tabular}{|r|}
$\begin{array}{r}\text { Vs30N } \\
\left(\mathbf{m ~ s}^{-1}\right)\end{array}$ \\
160.1
\end{tabular}} \\
\hline & & & & \multicolumn{2}{|c|}{ PGAh \pm sd } & \multicolumn{2}{|c|}{$\mathrm{PGVh} \pm \mathrm{sd}$} & \multicolumn{2}{|c|}{ PGAh \pm sd } & \multicolumn{2}{|c|}{ PGVh \pm sd } & & & & & \\
\hline & & & & 0.301 & 0.789 & 0.545 & 0.670 & 0.397 & 0.392 & 0.643 & 0.230 & & & & & \\
\hline 2 & TAP002 & 25.13 & 121.46 & -0.208 & 0.019 & -0.127 & 0.069 & 0.063 & 0.000 & 0.170 & 0.014 & 2 & D & 374.0 & $\mathrm{C}$ & 374.0 \\
\hline 3 & TAP003 & 25.09 & 121.45 & -0.641 & 0.092 & 0.025 & 0.117 & -0.371 & 0.030 & 0.322 & 0.039 & 2 & E & 209.4 & D & 209.4 \\
\hline 4 & TAP004 & 25.11 & 121.47 & -0.002 & 0.362 & 0.395 & 0.521 & 0.193 & 0.102 & 0.685 & 0.158 & 2 & E & 195.5 & $\mathrm{D}$ & 195.5 \\
\hline 5 & TAP005 & 25.11 & 121.51 & 0.326 & 0.000 & 0.340 & 0.000 & 0.465 & 0.000 & 0.492 & 0.000 & 1 & E & 177.0 & E & 177.0 \\
\hline 6 & TAP006 & 25.10 & 121.51 & 0.004 & 0.042 & 0.020 & 0.056 & 0.274 & 0.005 & 0.316 & 0.009 & 2 & E & 198.4 & D & 198.4 \\
\hline 7 & TAP007 & 25.08 & 121.51 & 0.322 & 0.474 & 0.455 & 0.508 & 0.463 & 0.193 & 0.612 & 0.157 & 5 & E & 204.3 & $\mathrm{D}$ & 204.3 \\
\hline 8 & TAP008 & 25.08 & 121.53 & -0.465 & 0.000 & -0.006 & 0.000 & -0.062 & 0.000 & 0.435 & 0.000 & 1 & $\mathrm{E}$ & 191.6 & D & 191.6 \\
\hline 9 & TAP009 & 25.08 & 121.57 & 0.115 & & 0.037 & 0.038 & & & & 0.003 & 2 & $\mathrm{E}$ & & D & 187.6 \\
\hline 10 & TAP010 & 25.07 & 121.47 & -0.438 & 0.207 & -0.019 & 0.135 & -0.167 & 0.104 & 0.278 & 0.049 & 2 & E & 217.5 & $\mathrm{D}$ & 217.5 \\
\hline 11 & TAP011 & 25.06 & 121.49 & -0.182 & 0.218 & -0.107 & 0.098 & 0.089 & 0.112 & 0.191 & 0.028 & 2 & D & 211.1 & D & 211.1 \\
\hline 12 & TAP012 & 25.06 & & & & & & & & & & 2 & E & & D & 207.4 \\
\hline 13 & TAP013 & 25.06 & 121.53 & 0.519 & 0.310 & 0.484 & 0.642 & 0.514 & 0.216 & 0.546 & 0.383 & 5 & E & 207.9 & $\mathrm{D}$ & 207.9 \\
\hline 14 & TAP014 & 25.06 & 121.54 & -0.105 & 0.092 & 0.259 & 0.012 & 0.166 & 0.029 & 0.556 & 0.001 & 2 & E & 192.1 & D & 192.1 \\
\hline 15 & TAP015 & 25.05 & & & & & & & & & & 4 & E & & D & 208.1 \\
\hline 16 & TAP016 & 25.06 & 121.42 & -0.582 & 0.000 & 0.166 & 0.000 & -0.444 & 0.000 & 0.318 & 0.000 & 1 & $\mathrm{E}$ & 326.6 & D & 326.6 \\
\hline 17 & TAP017 & 25.05 & 121.45 & 0.157 & 0.000 & 0.500 & 0.000 & 0.295 & 0.000 & 0.652 & 0.000 & 1 & $\mathrm{E}$ & 221.4 & D & 221.4 \\
\hline 18 & TAP019 & 25.04 & 121.49 & & & & & & & & & 2 & $\mathrm{E}$ & & D & 225.2 \\
\hline 19 & TAP020 & 25.04 & 121.53 & -0.264 & 0.110 & 0.086 & 0.089 & 0.007 & 0.040 & 0.383 & 0.023 & 2 & E & 224.2 & $\mathrm{D}$ & 224.2 \\
\hline 20 & TAP021 & 25.04 & 121.54 & 0.132 & 0.086 & -0.042 & 0.032 & 0.403 & 0.026 & 0.255 & 0.001 & 2 & E & 165.3 & $\mathrm{E}$ & 165.3 \\
\hline 21 & TAP022 & 25.03 & 121.56 & 0.514 & 0.080 & 0.427 & 0.081 & 0.729 & 0.068 & 0.693 & 0.035 & 3 & D & 181.0 & D & 181.0 \\
\hline 22 & TAP023 & 25.01 & 121.46 & 0.007 & 0.480 & -0.060 & 0.156 & 0.278 & 0.314 & 0.238 & 0.063 & 2 & D & $* * * * *$ & $*$ & 254.7 \\
\hline 23 & TAP024 & 25.02 & 121.47 & 0.315 & 0.000 & 0.298 & 0.000 & 0.453 & 0.000 & 0.451 & 0.000 & 1 & D & 187.8 & $\mathrm{D}$ & 187.8 \\
\hline 24 & TAP025 & 25.03 & 121.49 & -0.452 & 0.000 & -0.489 & 0.000 & -0.050 & 0.000 & -0.048 & 0.000 & 1 & $\mathrm{E}$ & 250.2 & $\mathrm{D}$ & 250.2 \\
\hline 25 & TAP026 & 25.02 & 121.50 & 0.246 & 0.000 & 0.298 & 0.000 & 0.384 & 0.000 & 0.450 & 0.000 & 1 & E & 200.6 & D & 200.6 \\
\hline 26 & TAP028 & 25.00 & 121.51 & -0.054 & 0.000 & 0.053 & 0.000 & 0.084 & 0.000 & 0.205 & 0.000 & 1 & D & $* * * * *$ & $*$ & 270.1 \\
\hline 27 & TAP029 & 25.01 & 121.53 & 0.095 & 0.255 & -0.082 & 0.490 & 0.095 & 0.188 & -0.039 & 0.356 & 6 & D & $* * * * *$ & $*$ & 419.2 \\
\hline 28 & TAP030 & 25.00 & 121.53 & -1.087 & 0.217 & -0.438 & 0.212 & -1.091 & 0.415 & -0.313 & 0.175 & 3 & D & $* * * * *$ & $*$ & 686.7 \\
\hline 29 & TAP031 & 25.02 & 121.54 & -0.057 & 0.000 & 0.059 & 0.000 & 0.081 & 0.000 & 0.211 & 0.000 & 1 & $\mathrm{D}$ & 222.0 & $\mathrm{D}$ & 222.0 \\
\hline
\end{tabular}
is then used as the source-to-site distance, $\mathrm{M}_{\mathrm{w}}$ is moment

Table 1. Station code, location, site classifications, ground motion total and intra-event residuals, measured and estimated Vs30 of the TSMIP stations.

Note: (1) STname = Station Name.

(2) Lat = Station Latitude.

(3) Long = Station Longitude.

(4) TotalRes $=$ Total Residual in ln unit.

(5) IntraRes = Intra-event Residual in ln unit.

(6) $P G A h=$ The mean of residual for horizontal component of peak ground acceleration.

(7) $P G V h=$ The mean of residual for horizontal component of peak ground velocity.

(8) $s d=$ standard deviation.

(9) $R=$ Number of Records.

(10) Vs30 = The average shear-wave velocity in the upper $30 \mathrm{~m}$ of sediments in $\mathrm{m} \mathrm{s}^{-1}$.

(11) Vs30N = The estimated Vs30 values from the relation between the intra-event residual and the Vs30 in m s.1.

$\dagger$ Determined by Lee et al. (2001), on the basis of surface geology and borehole data.

$¥$ Determined according to UBC1997. 
Table 1. (Continued)

\begin{tabular}{|c|c|c|c|c|c|c|c|c|c|c|c|c|c|c|c|c|}
\hline \multirow{3}{*}{$\begin{array}{r}\text { No. } \\
30\end{array}$} & \multirow{3}{*}{$\begin{array}{l}\text { Stname } \\
\text { TAP032 }\end{array}$} & \multirow{3}{*}{$\begin{array}{c}\begin{array}{c}\text { Lat } \\
\left({ }^{\circ} \mathbf{N}\right)\end{array} \\
25.00\end{array}$} & \multirow{3}{*}{$\begin{array}{c}\text { Long } \\
\left({ }^{\circ} \mathbf{E}\right)\end{array}$} & \multicolumn{4}{|c|}{ TotalRes } & \multicolumn{4}{|c|}{ IntraRes } & \multirow{3}{*}{$\begin{array}{c}\mathbf{R} \\
15\end{array}$} & \multirow{3}{*}{$\frac{\text { Site } \dagger}{D}$} & \multirow{3}{*}{$\begin{array}{c}\begin{array}{c}\text { Vs30 } \\
\left(\mathbf{m ~ s}^{-1}\right)\end{array} \\
314.9\end{array}$} & \multirow{3}{*}{$\frac{\text { Site } F}{D}$} & \multirow{3}{*}{$\begin{array}{r}\begin{array}{r}\text { Vs30N } \\
\left(\mathbf{m ~ s}^{-1}\right)\end{array} \\
314.9\end{array}$} \\
\hline & & & & \multicolumn{2}{|c|}{ PGAh \pm sd } & \multicolumn{2}{|c|}{$P G V h \pm s d$} & PGA & $\pm \mathrm{sd}$ & PGV & $\pm \mathrm{sd}$ & & & & & \\
\hline & & & & 0.414 & 0.378 & 0.353 & 0.249 & 0.461 & 0.240 & 0.430 & 0.229 & & & & & \\
\hline 31 & TAP033 & 24.98 & 121.53 & 0.268 & 363 & 0.199 & 311 & 0.322 & 0.207 & 0.236 & .272 & 12 & D & 486.3 & $\mathrm{C}$ & 486.3 \\
\hline 32 & TAP034 & 24.95 & 121.53 & 0.006 & 0.268 & -0.421 & 0.233 & 0.047 & 0.099 & -0.341 & 0.131 & 15 & B & & $*$ & 722.7 \\
\hline 33 & TAP035 & 24.92 & 121.54 & 0.233 & 0.340 & -0.230 & 0.253 & 0.238 & 0.252 & -0.207 & 0.204 & 21 & B & 404.7 & $\mathrm{C}$ & 404.7 \\
\hline 34 & TAP036 & 24.90 & 121.54 & -0.478 & 0.309 & -0.682 & 0.297 & -0.468 & 0.185 & -0.632 & 0.124 & 8 & B & $* * * * *$ & $*$ & 1219.9 \\
\hline 35 & TAP037 & 25.04 & 121.43 & 0.101 & 0.317 & 0.465 & 0.381 & -0.063 & 0.180 & 0.393 & 0.263 & 8 & D & 219.9 & D & 219.9 \\
\hline 36 & TAP038 & 25.02 & 121.41 & 0.485 & 0.460 & 0.634 & 0.348 & 0.464 & 0.342 & 0.593 & 0.189 & 7 & D & 209.0 & D & 209.0 \\
\hline 37 & TAP039 & 24.94 & 121.36 & -0.243 & 0.263 & -0.297 & 0.465 & -0.307 & 0.228 & -0.275 & 0.302 & 7 & D & 661.6 & $\mathrm{C}$ & 661.6 \\
\hline 38 & TAP040 & 25.18 & 121.43 & 0.369 & 0.347 & 0.249 & 0.537 & 0.306 & 0.248 & 0.255 & 0.414 & 10 & $\mathrm{C}$ & 432.5 & $\mathrm{C}$ & 432.5 \\
\hline 39 & TAP041 & 25.18 & 121.42 & 0.289 & 0.200 & 0.208 & 0.333 & 0.281 & 0.163 & 0.273 & 0.284 & 14 & D & 360.5 & $\mathrm{C}$ & 360.5 \\
\hline 40 & TAP042 & 25.13 & 121.45 & 0.126 & 0.116 & -0.055 & 0.054 & 0.074 & 0.064 & -0.064 & 0.127 & 12 & D & $*$ & $*$ & 438.2 \\
\hline 41 & TAP043 & 24.99 & 121.41 & 0.184 & 0.307 & -0.003 & 0.266 & 0.155 & 0.247 & 0.086 & 0.185 & 13 & D & 382.6 & $\mathrm{C}$ & 382.6 \\
\hline 42 & TAP044 & 24.98 & 121.39 & -0.222 & 0.347 & -0.101 & 0.330 & -0.215 & 0.190 & 0.006 & 0.182 & 7 & D & 473.0 & $\mathrm{C}$ & 473.0 \\
\hline 43 & TAP045 & 25.11 & 121.82 & -0.170 & 0.172 & -0.656 & 0.126 & -0.129 & 0.248 & -0.565 & 0.077 & 7 & B & 987.7 & B & 987.7 \\
\hline 44 & TAP046 & 25.10 & 121.77 & 0.061 & 0.126 & -0.391 & 0.097 & 0.060 & 0.054 & -0.311 & 0.030 & 15 & D & 822.0 & B & 822.0 \\
\hline 45 & TAP047 & 24.95 & 121.34 & -0.280 & 0.139 & -0.445 & 52 & -0.331 & 0.140 & -0.402 & 37 & 11 & D & 71.2 & $*$ & 571.2 \\
\hline 46 & TAP048 & 24.97 & 121.43 & -0.366 & 0.370 & -0.461 & 0.162 & -0.095 & 0.227 & -0.164 & 0.067 & 2 & D & $* * * * *$ & * & 525.0 \\
\hline 47 & TAP049 & 25.15 & 121.44 & 0.262 & 0.237 & 0.341 & 0.300 & 0.258 & 0.187 & 0.375 & 0.240 & 17 & $\mathrm{C}$ & $* * * *$ & * & 199.0 \\
\hline 48 & TAP050 & 25.15 & 121.40 & 0.524 & 0.509 & 0.598 & 0.583 & 0.494 & 0.334 & 0.573 & 0.356 & 6 & D & 280.7 & $\mathrm{D}$ & 280.7 \\
\hline 49 & TAP051 & 25.10 & 121.44 & -0.059 & 0.105 & -0.070 & 0.151 & -0.125 & 0.071 & -0.095 & 0.168 & 13 & B & 401.8 & $\mathrm{C}$ & 401.8 \\
\hline 50 & TAP052 & 25.08 & 121.38 & 0.276 & 0.165 & -0.125 & 0.245 & 0.198 & 0.0 & -0.112 & 0.233 & 13 & $\mathrm{C}$ & 34.4 & $\mathrm{C}$ & 434.4 \\
\hline 51 & TAP053 & 24.96 & 121.52 & 0.160 & 0.353 & -0.119 & & & 0.227 & -0.053 & 0.175 & 10 & D & $* *$ & 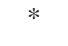 & 429.6 \\
\hline 52 & TAP054 & 25.00 & 121.43 & 0.245 & 0.185 & 0.501 & 0.221 & 0.267 & 0.233 & 0.683 & 0.184 & 3 & $\mathrm{D}$ & 309.7 & $\mathrm{D}$ & 309.7 \\
\hline 53 & TAP055 & 25.17 & 121.44 & 0.368 & .271 & 0.439 & 0.511 & 0.336 & 0.201 & 0.442 & 0.335 & 6 & D & & 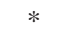 & 176.1 \\
\hline 54 & TAP056 & 25.19 & 121.52 & & 0.000 & -0.021 & & & 0.000 & & 0.000 & 1 & $\mathrm{C}$ & 590.1 & $\mathrm{C}$ & 590.1 \\
\hline 55 & TAP057 & 25.08 & 121.64 & 0.312 & 0.155 & -0.191 & 0.073 & 0.426 & 0.064 & 0.047 & 0.045 & 5 & D & 366.6 & $\mathrm{C}$ & 366.6 \\
\hline 56 & TAP058 & 25.13 & 121.71 & -0.506 & 0.168 & -0.432 & 0.177 & -0.544 & 0.152 & -0.434 & 78 & 6 & B & 1056.7 & & 1056.7 \\
\hline 57 & TAP059 & 25.16 & 121.69 & -0.212 & 0.096 & -0.552 & 0.156 & -0.223 & 0.132 & -0.496 & 0.141 & 15 & B & 450.6 & $\mathrm{C}$ & 450.6 \\
\hline 58 & TAP060 & 25.16 & 121.72 & -0.294 & 0.133 & -0.585 & 0.178 & -0.313 & 0.134 & -0.479 & 0.108 & 12 & B & $* * * *$ & $*$ & 926.3 \\
\hline 59 & TAP061 & 25.13 & 121.75 & & & & & & & & & 9 & B & & 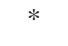 & 630.3 \\
\hline 60 & TAP062 & 25.13 & 121.78 & -0.261 & 0.037 & -0.534 & 50 & -0.216 & 0.0 & -0.3 & 0.016 & 6 & B & ****** & $*$ & 727.1 \\
\hline 61 & TAP063 & 25.10 & 121.73 & 0.188 & 0.024 & -0.272 & 0.174 & 0.169 & 0.029 & -0.245 & 0.040 & 6 & B & $* * * * *$ & * & 607.3 \\
\hline 62 & TAP064 & 25.15 & 121.77 & -0.287 & 0.020 & -0.59 & 0.020 & -0.017 & 0.000 & -0.298 & 0.000 & 2 & B & $* * * * *$ & $*$ & 668.4 \\
\hline 63 & TAP065 & 25.15 & 121.77 & -0.697 & 0.050 & -0.617 & 0.155 & -0.742 & 0.051 & -0.510 & 0.080 & 8 & B & 1034.7 & & 1034.7 \\
\hline 64 & TAP066 & 25.19 & 121.52 & -0.676 & 0.200 & -0.698 & 0.143 & -0.621 & 0.103 & -0.591 & 0.073 & 12 & $\mathrm{C}$ & 658.0 & $\mathrm{C}$ & 658.0 \\
\hline 65 & TAP067 & 24.98 & 121.58 & -0.382 & 0.215 & -0.281 & 0.241 & -0.436 & 0.131 & -0.244 & & 12 & B & 815.0 & B & 815.0 \\
\hline 66 & TAP068 & 24.88 & 121.53 & -0.356 & 0.155 & -0.692 & 0.100 & -0.086 & 0.068 & -0.395 & 0.029 & 2 & B & $* * * * *$ & $*$ & 796.1 \\
\hline 67 & TAP069 & 25.01 & 121.99 & -0.626 & 0.317 & -0.543 & 0.239 & -0.797 & 0.489 & -0.645 & 0.329 & 6 & B & $* * * *$ & $*$ & 1249.7 \\
\hline 68 & & 25.12 & 121.91 & -0.534 & 0.147 & -0.876 & & & & -0.702 & & 5 & B & $* * * * *$ & $*$ & 1384.1 \\
\hline 69 & TAP071 & 25.00 & 121.61 & 0.069 & 0.320 & -0.128 & 0.331 & 0.103 & 0.188 & -0.086 & 0.135 & 9 & D & 831.4 & B & 831.4 \\
\hline 70 & TAP072 & 24.99 & 121.65 & -0.103 & 0.229 & -0.481 & 0.323 & -0.198 & 0.143 & -0.435 & 0.192 & 8 & B & $* * * *$ & * & 855.7 \\
\hline 71 & TAP073 & 25.01 & 121.68 & -0.119 & 0.171 & -0.546 & & -0.002 & 0.143 & -0.299 & 0.205 & 4 & B & $* * * * *$ & $*$ & 669.6 \\
\hline 72 & TAP074 & 24.95 & 121.63 & 0.118 & 0.025 & -0.017 & 0.080 & 0.031 & 0.156 & -0.115 & 0.084 & 4 & B & $* * * * *$ & $*$ & 480.4 \\
\hline 73 & TAP075 & 25.03 & 121.73 & 0.020 & 0.164 & -0.247 & 0.216 & 0.001 & 0.078 & -0.204 & 0.127 & 15 & B & 851.1 & B & 851.1 \\
\hline 74 & TAP077 & 25.06 & 121.84 & -0.266 & 0.047 & -0.267 & 0.114 & -0.315 & 0.05 & -0.250 & 0.045 & 9 & D & 992.4 & B & 992.4 \\
\hline 75 & TAP078 & 25.06 & 121.87 & 0.062 & 0.176 & -0.059 & 0.230 & 0.017 & 0.112 & -0.022 & 0.125 & 8 & B & $* * * * *$ & $*$ & 406.1 \\
\hline 76 & TAP079 & 25.02 & 121.91 & -0.392 & 0.136 & -0.536 & 0.150 & -0.502 & 0.147 & -0.551 & 0.158 & 9 & B & $* * * * * *$ & $*$ & 1055.0 \\
\hline 77 & TAP080 & 25.02 & 121.94 & 0.512 & 0.155 & -0.139 & 0.239 & 0.362 & 0.066 & -0.194 & 0.131 & 7 & D & 401.8 & $\mathrm{C}$ & 401.8 \\
\hline 78 & TAP081 & 25.02 & 121.98 & -0.723 & 0.035 & -0.712 & 0.055 & -0.847 & 0.164 & -0.665 & 0.121 & 6 & B & $* * * * *$ & $*$ & 1295.6 \\
\hline 79 & TAP082 & 25.06 & 121.92 & -0.762 & 0.162 & -0.799 & 0.228 & -0.813 & 0.072 & -0.791 & 0.116 & 5 & D & $* * * * *$ & $*$ & 1624.3 \\
\hline 80 & TAP083 & 25.26 & 121.49 & -0.539 & 0.057 & -0.401 & 0.129 & -0.650 & 0.105 & -0.414 & 0.147 & 9 & D & ****** & $*$ & 823.5 \\
\hline 81 & TAP084 & 25.23 & 121.63 & -0.153 & 0.125 & -0.330 & 0.211 & -0.151 & 0.159 & -0.274 & 0.192 & 13 & D & 204.1 & D & 204.1 \\
\hline 82 & TAP085 & 24.94 & 121.71 & -0.187 & 0.169 & -0.375 & 0.357 & -0.133 & 0.012 & -0.312 & 0.036 & 3 & B & 895.1 & B & 895.1 \\
\hline
\end{tabular}


Table 1. (Continued)

\begin{tabular}{|c|c|c|c|c|c|c|c|c|c|c|c|c|c|c|c|c|}
\hline \multirow{3}{*}{$\begin{array}{r}\text { No. } \\
83\end{array}$} & \multirow{3}{*}{$\begin{array}{l}\text { Stname } \\
\text { TAP086 }\end{array}$} & \multirow{3}{*}{$\begin{array}{c}\begin{array}{c}\text { Lat } \\
\left({ }^{\circ} \mathbf{N}\right)\end{array} \\
24.95\end{array}$} & \multirow{3}{*}{$\begin{array}{c}\text { Long } \\
\left({ }^{\circ} \mathbf{E}\right)\end{array}$} & \multicolumn{4}{|c|}{ TotalRes } & \multicolumn{4}{|c|}{ IntraRes } & \multirow{3}{*}{$\begin{array}{c}\mathbf{R} \\
13\end{array}$} & \multirow{3}{*}{$\frac{\text { Site } \dagger}{\mathrm{B}}$} & \multirow{3}{*}{$\begin{array}{c}\begin{array}{c}\text { Vs30 } \\
\left(\mathbf{m ~ s}^{-1}\right)\end{array} \\
942.8\end{array}$} & \multirow{3}{*}{$\frac{\text { Site } F}{\text { B }}$} & \multirow{3}{*}{$\begin{array}{r}\begin{array}{r}\text { Vs30N } \\
\left(\mathbf{m ~ s}^{-1}\right)\end{array} \\
942.8\end{array}$} \\
\hline & & & & \multicolumn{2}{|c|}{ PGAh \pm sd } & \multicolumn{2}{|c|}{$P G V h \pm s d$} & PGA & $\pm \mathrm{sd}$ & PGV & $\pm \mathrm{sd}$ & & & & & \\
\hline & & & & -0.182 & 0.348 & -0.408 & 0.221 & -0.127 & 0.137 & -0.339 & 0.125 & & & & & \\
\hline 84 & TAP087 & 25.10 & 121.42 & -0.306 & .347 & -0.202 & 447 & -0.317 & 0.257 & -0.116 & .306 & 11 & $\mathrm{C}$ & $* * * *$ & 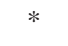 & 481.1 \\
\hline 85 & TAP088 & 25.04 & 121.59 & -0.040 & 0.005 & -0.187 & 0.006 & 0.175 & 0.067 & 0.008 & 0.030 & 2 & D & 228.0 & $\mathrm{D}$ & 228.0 \\
\hline 86 & TAP089 & 25.03 & 121.57 & -0.869 & 0.000 & -1.149 & 0.000 & -0.466 & 0.000 & -0.707 & 0.000 & 1 & D & 442.1 & $\mathrm{C}$ & 442.1 \\
\hline 87 & TAP090 & 25.06 & 121.60 & -0.213 & 0.000 & -0.081 & 0.000 & 0.190 & 0.000 & 0.361 & 0.000 & 1 & $\mathrm{E}$ & 324.6 & D & 324.6 \\
\hline 88 & TAP091 & 25.06 & 121.59 & -0.081 & 0.000 & -0.212 & 0.000 & 0.322 & 0.000 & 0.230 & 0.000 & 1 & E & 177.3 & E & 177.3 \\
\hline 89 & TAP092 & 25.07 & 121.56 & -0.284 & 0.000 & -0.386 & 0.000 & 0.119 & 0.000 & 0.056 & 0.000 & 1 & $\mathrm{E}$ & $* * * * *$ & * & 353.2 \\
\hline 90 & TAP093 & 25.09 & 121.56 & 0.685 & 0.348 & 0.698 & 0.422 & 0.732 & 0.183 & 0.817 & 0.203 & 5 & $\mathrm{E}$ & 193.6 & D & 193.6 \\
\hline 91 & TAP094 & 25.14 & 121.48 & -0.709 & 0.000 & -0.538 & 0.000 & -0.306 & 0.000 & -0.097 & 0.000 & 1 & D & 409.9 & $\mathrm{C}$ & 409.9 \\
\hline 92 & TAP095 & 25.14 & 121.49 & -0.653 & 0.000 & -0.549 & 0.000 & -0.251 & 0.000 & -0.107 & 0.000 & 1 & $\mathrm{E}$ & 205.9 & D & 205.9 \\
\hline 93 & TAP097 & 25.02 & 121.53 & 0.265 & 0.262 & 0.318 & 0.326 & 0.269 & 0.198 & 0.309 & 0.203 & 14 & E & $* * * * *$ & $*$ & 223.9 \\
\hline 94 & TAP098 & 25.10 & 121.54 & -0.265 & 0.000 & -0.445 & 0.000 & 0.137 & 0.000 & -0.004 & 0.000 & 1 & $\mathrm{C}$ & $* * * * *$ & $*$ & 393.5 \\
\hline 95 & TAP099 & 25.04 & 121.51 & -0.465 & 0.000 & -0.347 & 0.000 & -0.063 & 0.000 & 0.094 & 0.000 & 1 & $\mathrm{E}$ & $* * * * *$ & $*$ & 329.8 \\
\hline 96 & TAP100 & 25.03 & 121.52 & 0.403 & 0.499 & 0.341 & 0.677 & 0.479 & 0.348 & 0.509 & 0.438 & 5 & $\mathrm{E}$ & $* * * * *$ & $*$ & 156.0 \\
\hline 97 & TAP103 & 25.07 & 121.78 & 0.577 & 0.197 & 0.481 & 0.195 & 0 & 0.1 & 0.486 & 0.168 & 15 & B & 444.9 & $\mathrm{C}$ & 444.9 \\
\hline 98 & TCU001 & 24.98 & 121.10 & 6 & 0.234 & 0 & 86 & 13 & 0 . & -0.095 & 085 & 3 & $\mathrm{C}$ & 570.2 & $\mathrm{C}$ & 570.2 \\
\hline 99 & TCU002 & 25.04 & 121.07 & -0.033 & 0.091 & 0.349 & 0.248 & -0.091 & 0.034 & 0.222 & 0.178 & 3 & $\mathrm{C}$ & 502.1 & $\mathrm{C}$ & 502.1 \\
\hline 100 & TCU003 & 25.05 & 121.14 & -0.062 & 0.191 & -0.045 & 0.229 & & 0.213 & -0.013 & 0.227 & 13 & D & 507.2 & $\mathrm{C}$ & 507.2 \\
\hline 101 & TCU004 & 25.06 & 121.19 & 0.137 & 0.241 & 0.260 & 0.518 & -0.072 & 0.378 & 0.186 & 0.422 & 2 & $\mathrm{C}$ & 518.8 & $\mathrm{C}$ & 3.8 \\
\hline 102 & TCU005 & 25.11 & 121.24 & -0.306 & 0.185 & -0.170 & 0.244 & -0.381 & 0.120 & -0.174 & 0.229 & 9 & D & 491.2 & $\mathrm{C}$ & 491.2 \\
\hline 103 & TCU006 & 24.91 & 121.14 & 0.016 & 0.207 & -0.028 & 0.137 & -0 . & 0 & -0.086 & 0.104 & 13 & $\mathrm{D}$ & 612.1 & $\mathrm{C}$ & 612.1 \\
\hline 104 & TCU007 & 25.00 & 121.31 & & 0.348 & & & & & & 0.170 & 16 & D & 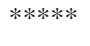 & * & .3 \\
\hline 105 & TCU008 & 25.01 & 121.21 & -0.136 & 0.139 & -0.206 & 0.064 & -0.069 & 0.066 & -0.157 & 0.140 & 9 & $\mathrm{C}$ & $* * * * *$ & $*$ & 518.2 \\
\hline 106 & TCU009 & 24.97 & 121.22 & -0.169 & 0.448 & 0.228 & 0.488 & -0.188 & 0.272 & & 0.269 & 6 & $\mathrm{C}$ & 66.0 & $\mathrm{C}$ & 466.0 \\
\hline 107 & TCU010 & 25.00 & 121.15 & -0.166 & 0.033 & -0.098 & 0.058 & -0.223 & & -0.068 & 0.112 & 8 & $\mathrm{C}$ & **** & $*$ & 441.6 \\
\hline 108 & TCU011 & 24.89 & 121.28 & -0.155 & 0.192 & -0.264 & 0.295 & -0.168 & 0.154 & -0.118 & 0.249 & 8 & D & 811.1 & B & 811.1 \\
\hline 109 & TCU012 & 24.94 & 121.29 & -0.061 & 0.254 & 0.013 & 0.280 & -0.124 & & 0.036 & 69 & 7 & $\mathrm{C}$ & 44.8 & $\mathrm{C}$ & 644.8 \\
\hline 110 & TCU013 & 24.87 & 121.20 & 0.195 & 0.228 & 0.011 & 0.274 & 0.131 & 0.152 & 0.034 & 0.163 & 7 & $\mathrm{C}$ & $* * * * *$ & $*$ & 367.6 \\
\hline 111 & TCU014 & 25.05 & 121.31 & 0.113 & 0.333 & 0.059 & 0.396 & 0.163 & 0.175 & 0.119 & 0.276 & 15 & D & 523.2 & $\mathrm{C}$ & 523.2 \\
\hline 112 & TCU015 & 24.76 & & & & & & & & & & 15 & $\mathrm{C}$ & & $\mathrm{C}$ & \\
\hline 113 & TCU016 & 24.82 & 120.96 & & 0 & 0.094 & & & & & 71 & 6 & D & 485.5 & $\mathrm{C}$ & 485.5 \\
\hline 114 & TCU017 & 24.78 & 121.01 & -0.145 & 0.278 & 0.069 & 0.172 & -0.135 & 0.156 & 0.106 & 0.112 & 12 & $\mathrm{C}$ & 548.3 & $\mathrm{C}$ & 548.3 \\
\hline 115 & TCU018 & 24.88 & 121.04 & & & & 0.204 & -0.257 & & -0.103 & & 12 & $\mathrm{C}$ & 年 & 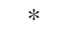 & \\
\hline 116 & TCU019 & 24.90 & 120.98 & 0.241 & 0.137 & 0.306 & 0.121 & 0.295 & 0.076 & 0.370 & 0.054 & 3 & $\mathrm{C}$ & 506.0 & $\mathrm{C}$ & 506.0 \\
\hline 117 & TCU020 & 24.84 & 121.00 & 0.737 & 0.000 & 0.598 & 0.000 & 0.649 & 0.000 & 0.563 & 0.000 & 1 & D & 489.0 & $\mathrm{C}$ & 489.0 \\
\hline 118 & TCU021 & 24.79 & 121.17 & & 0.338 & -0.062 & & & 0.342 & -0.138 & & 6 & D & 446.4 & $\mathrm{C}$ & 446.4 \\
\hline 119 & TCU022 & 24.77 & 120.98 & -0.191 & 0.001 & -0.019 & 0.006 & -0.165 & 0.008 & -0.016 & 0.052 & 2 & D & 436.7 & $\mathrm{C}$ & 436.7 \\
\hline 120 & TCU023 & 24.72 & 121.13 & -0.156 & 0.000 & -0.219 & 0.000 & -0.018 & 0.000 & -0.067 & 0.000 & 1 & $\mathrm{C}$ & 555.2 & $\mathrm{C}$ & 555.2 \\
\hline 121 & TCU024 & 24.74 & 121.08 & & & & & & & & & 11 & $\mathrm{C}$ & 383.2 & $\mathrm{C}$ & 383.2 \\
\hline 122 & TCU025 & 24.71 & 121.18 & -0.556 & 0.321 & -0.892 & 0.348 & -0.582 & 0.161 & -0.792 & 0.181 & 9 & B & **** & $*$ & 1626.9 \\
\hline 123 & TCU026 & 24.78 & 121.08 & 0.037 & 0.338 & 0.060 & 0.122 & 0.042 & 0.208 & 0.073 & 0.134 & 17 & D & 606.5 & $\mathrm{C}$ & 606.5 \\
\hline 124 & TCU027 & 24.83 & 121.06 & -0.137 & & -0.038 & & -0.209 & 0.184 & -0.043 & & 7 & D & 670.4 & $\mathrm{C}$ & 670.4 \\
\hline 125 & TCU028 & 24.70 & 121.04 & 0.494 & 0.362 & 0.207 & 0.345 & 0.511 & 0.306 & 0.269 & 0.334 & 6 & D & 524.9 & $\mathrm{C}$ & 524.9 \\
\hline 126 & TCU029 & 24.56 & 120.75 & 0.154 & 0.392 & & & & 0.206 & -0.154 & 0.135 & 14 & $\mathrm{C}$ & 404.8 & $\mathrm{C}$ & 404.8 \\
\hline 127 & TCU030 & 24.59 & 120.88 & -0.089 & & -0.163 & 0.373 & -0.10 & 0.27 & -0.173 & 0.317 & 9 & D & 624.7 & $\mathrm{C}$ & 624.7 \\
\hline 128 & TCU031 & 24.56 & 120.70 & 0.088 & 0.209 & 0.150 & 0.116 & 0.071 & 0.179 & 0.143 & 0.120 & 18 & $\mathrm{C}$ & 476.3 & $\mathrm{C}$ & 476.3 \\
\hline 129 & TCU032 & 24.52 & 120.81 & -0.221 & 0.186 & -0.029 & 0.108 & -0.206 & 0.131 & -0.074 & 0.085 & 11 & D & 500.3 & $\mathrm{C}$ & 500.3 \\
\hline 130 & TCU033 & 24.69 & 120.86 & & 0.263 & 0.154 & 0.171 & 0.400 & 0.167 & 0.149 & 0.165 & 19 & D & 420.6 & $\mathrm{C}$ & 420.6 \\
\hline 131 & TCU034 & 24.64 & 120.86 & 0.175 & 0.426 & 0.067 & 0.280 & 0.207 & 0.265 & 0.103 & 0.209 & 15 & $\mathrm{C}$ & 391.6 & $\mathrm{C}$ & 391.6 \\
\hline 132 & TCU035 & 24.62 & 120.79 & 0.028 & 0.618 & 0.094 & 0.451 & 0.110 & 0.493 & 0.147 & 0.451 & 13 & D & 378.1 & $\mathrm{C}$ & 378.1 \\
\hline 133 & TCU036 & 24.45 & 120.70 & -0.035 & 0.402 & -0.036 & 0.195 & -0.003 & 0.200 & -0.009 & 0.139 & 24 & D & 483.1 & $\mathrm{C}$ & 483.1 \\
\hline 134 & TCU037 & 24.49 & 120.68 & -0.078 & 0.259 & -0.120 & 0.312 & -0.118 & 0.161 & -0.150 & 0.237 & 8 & D & 463.5 & $\mathrm{C}$ & 463.5 \\
\hline 135 & TCU038 & 24.49 & 120.66 & 0.221 & 0.350 & 0.094 & 0.225 & 0.242 & 0.160 & 0.122 & 0.188 & 25 & D & $* * * * *$ & $*$ & 313.6 \\
\hline
\end{tabular}


Table 1. (Continued)

\begin{tabular}{|c|c|c|c|c|c|c|c|c|c|c|c|c|c|c|c|c|}
\hline \multirow{3}{*}{$\begin{array}{l}\text { No. } \\
136\end{array}$} & \multirow{3}{*}{$\begin{array}{c}\text { Stname } \\
\text { TCU039 }\end{array}$} & \multirow{3}{*}{$\begin{array}{c}\begin{array}{c}\text { Lat } \\
\left({ }^{\circ} \mathbf{N}\right)\end{array} \\
24.49\end{array}$} & \multirow{3}{*}{$\begin{array}{c}\begin{array}{c}\text { Long } \\
\left({ }^{\circ} \mathbf{E}\right)\end{array} \\
120.78\end{array}$} & \multicolumn{4}{|c|}{ TotalRes } & \multicolumn{4}{|c|}{ IntraRes } & \multirow{3}{*}{$\begin{array}{l}\mathbf{R} \\
16\end{array}$} & \multirow{3}{*}{$\frac{\text { Site } \dagger}{\mathrm{C}}$} & \multirow{3}{*}{$\begin{array}{c}\begin{array}{c}\text { Vs30 } \\
\left(\mathbf{m ~ s}^{-1}\right)\end{array} \\
549.6\end{array}$} & \multirow{3}{*}{\begin{tabular}{|c|} 
Site $\$$ \\
C
\end{tabular}} & \multirow{3}{*}{$\begin{array}{r}\begin{array}{r}\text { Vs30N } \\
\left(\mathbf{m ~ s}^{-1}\right)\end{array} \\
549.6\end{array}$} \\
\hline & & & & \multicolumn{2}{|c|}{ PGAh \pm sd } & \multicolumn{2}{|c|}{$P G V h \pm s d$} & PGAh & $\pm \mathrm{sd}$ & PGV & $\pm \mathrm{sd}$ & & & & & \\
\hline & & & & 0.197 & 0.387 & 0.236 & 0.105 & 0.218 & 0.172 & 0.242 & 0.115 & & & & & \\
\hline 137 & TCU040 & 24.45 & 120.64 & 0.212 & 42 & 0.139 & 173 & 0.248 & 185 & 0.178 & 150 & 23 & E & 36.2 & $\mathrm{C}$ & 336.2 \\
\hline 138 & TCU041 & 24.39 & 120.69 & 0.001 & 0.245 & -0.046 & 0.300 & -0.035 & & -0.043 & & 9 & D & & $*$ & 421.9 \\
\hline 139 & TCU042 & 24.55 & 120.81 & 0.290 & 0.450 & 0.100 & 0.214 & 0.306 & 0.257 & 0.129 & 0.183 & 25 & $\mathrm{D}$ & 73.5 & $\mathrm{C}$ & 573.5 \\
\hline 140 & TCU043 & 24.69 & 120.95 & 0.254 & 0.233 & -0.138 & 0.344 & 0.179 & 0.173 & -0.117 & .319 & 10 & $\mathrm{C}$ & 540.0 & $\mathrm{C}$ & 540.0 \\
\hline 141 & TCU044 & 24.40 & 120.76 & 0.059 & & & & -0.174 & & & & 8 & D & 508.4 & $\mathrm{C}$ & 508.4 \\
\hline 142 & TCU045 & 24.54 & 120.91 & -0.248 & 0.311 & -0.534 & 0.122 & -0.259 & 0.197 & -0.545 & & 15 & $\mathrm{C}$ & 707.0 & $\mathrm{C}$ & 707.0 \\
\hline 143 & TCU046 & 24.47 & 120.85 & -0.489 & 0.296 & -0.510 & 0.205 & -0.422 & 0.100 & -0.443 & .192 & 17 & B & 462.6 & $\mathrm{C}$ & 462.6 \\
\hline 144 & TCU047 & 24.62 & 120.94 & 0.003 & 0.672 & -0.197 & 0.653 & 0.070 & 0.524 & -0.153 & 0.462 & 13 & $\mathrm{C}$ & 523.0 & $\mathrm{C}$ & 523.0 \\
\hline 145 & TCU048 & 24.18 & 120.59 & 0.058 & 0.409 & -0.236 & 0.194 & 0.012 & 0.200 & -0.248 & 0.208 & 22 & $\mathrm{C}$ & 558.4 & $\mathrm{C}$ & 558.4 \\
\hline 146 & TCU049 & 24.18 & 120.69 & -0.220 & 0.269 & & & -0.228 & & & & 21 & D & 59.7 & $\mathrm{C}$ & 469.7 \\
\hline 147 & TCU050 & 24.18 & 120.63 & -0.178 & 0.383 & -0.172 & 0.253 & -0.217 & 0.221 & -0.195 & 0.251 & 19 & D & 539.3 & $\mathrm{C}$ & 539.3 \\
\hline 148 & TCU051 & 24.16 & 120.65 & 0.006 & 0.376 & -0.031 & 0.181 & 0.002 & 0.196 & -0.063 & 0.206 & 18 & D & & $*$ & 437.9 \\
\hline 149 & TCU052 & 24.20 & 120.74 & -0.191 & & & & & & & & 24 & $\mathrm{C}$ & 89.2 & $\mathrm{C}$ & 589.2 \\
\hline 150 & TCU053 & 24.19 & 120.67 & -0.310 & 0.437 & -0.261 & 0.191 & -0.313 & 0.253 & -0.285 & 0.218 & 18 & $\mathrm{D}$ & 452.1 & $\mathrm{C}$ & 452.1 \\
\hline 151 & TCU054 & 24.16 & 120.68 & -0.387 & 0.346 & -0.228 & 0.256 & -0.263 & 0.128 & -0.157 & & 11 & D & 437.0 & $\mathrm{C}$ & 437.0 \\
\hline 152 & TCU055 & 24.14 & 120.66 & 0 . & 0. & 0 & 0.212 & 0.288 & 0.052 & & & 10 & $\mathrm{D}$ & $* * * * *$ & $*$ & 303.9 \\
\hline 153 & TCU056 & 24.16 & 120.62 & -0.042 & 0.343 & -0.102 & 0.266 & -0.049 & 0.174 & -0.092 & 77 & 21 & $\mathrm{D}$ & 401.2 & $\mathrm{C}$ & 401.2 \\
\hline 154 & & & 120.61 & -0.209 & & -0.084 & 0.174 & -0.229 & 0.157 & -0.116 & 33 & 20 & $\mathrm{C}$ & & $*$ & 481.5 \\
\hline 155 & TCU058 & 24.25 & 120.70 & -0.300 & 0.2 & -0.281 & 0.034 & -0.337 & & -0.3 & & 5 & $\mathrm{D}$ & $* * * * *$ & $*$ & 694.9 \\
\hline 156 & TCU059 & 24.27 & 120.56 & & & & 0.295 & 0.542 & & & & 24 & D & 232.2 & $\mathrm{D}$ & 232.2 \\
\hline 157 & & & & & & & & & & & & 19 & 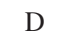 & & 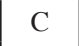 & \\
\hline 158 & TCU061 & 24.14 & 120.55 & 0.167 & 0.424 & 0.253 & 0.152 & 0.204 & 0.242 & 0.260 & 0.140 & 23 & $\mathrm{D}$ & 365.3 & $\mathrm{C}$ & 365.3 \\
\hline 159 & TCU062 & 24.12 & 120.67 & -0.107 & 0.352 & -0.140 & 0.294 & -0.169 & 0.218 & -0.111 & 0.231 & 9 & D & 451.0 & $\mathrm{C}$ & 451.0 \\
\hline 160 & & & & & & & & & & & & 17 & 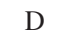 & & 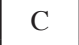 & \\
\hline 161 & TCU064 & 24.35 & 120.61 & -0.447 & 0.499 & -0.139 & 0.179 & -0.447 & 0.165 & -0.1 & 71 & 15 & D & 647.0 & $\mathrm{C}$ & 647.0 \\
\hline 162 & TCU065 & 24.06 & 120.69 & 0.483 & 0.373 & 0.429 & 0.435 & 0.636 & 0.262 & 0.573 & 0.398 & 19 & D & 290.1 & D & 290.1 \\
\hline 163 & & & & & & & & & & & & 5 & 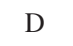 & & 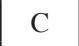 & \\
\hline 164 & $\mathrm{TC}$ & 24.09 & 120.72 & 7 & 0 & 0 & 0. & 0.112 & 0. & 0 . & & 16 & $\mathrm{D}$ & 44 & $\mathrm{C}$ & 440.3 \\
\hline 165 & TCU068 & 24.28 & 120.77 & -0.514 & 0.398 & -0.247 & 0.205 & -0.432 & 0.232 & -0.215 & 48 & 15 & D & 490.0 & $\mathrm{C}$ & 490.0 \\
\hline 166 & & & & & & & & & & & & 4 & D & & $\mathrm{C}$ & \\
\hline 167 & TCU070 & 24.20 & 120.55 & 0.375 & 0.560 & 0.473 & 0.303 & 0.411 & 0.346 & 0.415 & & 12 & $\mathrm{C}$ & 396.5 & $\mathrm{C}$ & 396.5 \\
\hline 168 & TCU071 & 23.99 & 120.79 & 0.008 & 0.476 & -0.161 & 0.327 & 0.035 & 0.799 & -0.107 & 0.515 & 15 & D & 614.8 & $\mathrm{C}$ & 614.8 \\
\hline 169 & TCU072 & 24.04 & 120.86 & -0.324 & 0.172 & -0.480 & 0.249 & -0.225 & 0.284 & -0.439 & & 16 & D & 471.9 & $\mathrm{C}$ & 471.9 \\
\hline 170 & TCU073 & 24.01 & 120.95 & -0.580 & 0.163 & -0.832 & 0.158 & -0.502 & 0.216 & -0.670 & & 10 & $\mathrm{D}$ & $* * * * *$ & $*$ & 1307.5 \\
\hline 171 & & & & & & & & & & & & 10 & D & & $\mathrm{C}$ & 8.2 \\
\hline 172 & TCU075 & 23.98 & 120.68 & & & & & & & & & 14 & D & & $\mathrm{C}$ & 521.2 \\
\hline 173 & TCU076 & 23.91 & 120.68 & 0.042 & 0.210 & -0.205 & 0.381 & 0.107 & 0.373 & -0.154 & 0.339 & 18 & $\mathrm{D}$ & 573.2 & $\mathrm{C}$ & 573.2 \\
\hline 174 & & & & & & & & & & & & 9 & D & & $\mathrm{C}$ & 7.5 \\
\hline 175 & TCU078 & 23.81 & 120.85 & & & -0.035 & & & & & & 10 & D & & $\mathrm{C}$ & 444.5 \\
\hline 176 & TCU079 & 23.84 & 120.89 & -0.130 & 0.569 & -0.305 & 0.492 & -0.017 & 0.772 & -0.254 & 0.587 & 13 & $\mathrm{D}$ & 353.9 & D & 353.9 \\
\hline 177 & TCU080 & 23.90 & 120.93 & & 0.415 & -0.326 & 0.220 & -0.179 & 0.309 & -0.410 & 0.186 & 7 & D & & $*$ & 818.0 \\
\hline 178 & TCU081 & 24.80 & 120.97 & -0.017 & 0163 & 0.132 & 0.117 & -0.071 & 0.070 & 0.108 & 0.103 & 14 & D & 427.1 & $\mathrm{C}$ & 427.1 \\
\hline 179 & TCU082 & 24.15 & 120.68 & -0.265 & 0.245 & -0.146 & 0.275 & -0.285 & 0.160 & -0.178 & 0.273 & 20 & $\mathrm{D}$ & 469.4 & $\mathrm{C}$ & 469.4 \\
\hline 180 & TCU083 & 24.97 & 121.19 & & & 0.050 & 0.297 & -0.015 & 0.172 & 0.081 & 0.236 & 13 & $\mathrm{C}$ & 370.7 & $\mathrm{C}$ & 370.7 \\
\hline 181 & TCU084 & 23.88 & 120.90 & -0.354 & 0200 & 0.102 & 0.262 & -0.318 & 0.220 & 0.120 & 0.257 & 19 & B & $* * * * *$ & $*$ & 315.0 \\
\hline 182 & TCU085 & 24.68 & 121.36 & -0.573 & 0.318 & -1.020 & 0.271 & -0.547 & 0.197 & -0.964 & 0.192 & 16 & B & & & 1037.9 \\
\hline 183 & TCU086 & 23.86 & 120.28 & & 0.522 & 0.224 & 0.511 & 0.306 & 0.327 & 0.169 & 0.364 & 15 & E & 222.9 & D & 222.9 \\
\hline 184 & TCU087 & 24.35 & 120.77 & -0.385 & 0.361 & -0.386 & 0.155 & -0.363 & 0.142 & -0.349 & 0.122 & 20 & B & $* * * * *$ & $*$ & 733.0 \\
\hline 185 & TCU088 & 24.25 & 121.18 & & & -0.929 & 0.269 & & & -0.779 & & 12 & B & & $*$ & 1590.1 \\
\hline 186 & TCU089 & 23.90 & 120.86 & -0.946 & 0.129 & -0.795 & 0.218 & -0.746 & 0.231 & -0.669 & 0.200 & 10 & B & $* * * * *$ & $*$ & 1304.5 \\
\hline 187 & TCU090 & 23.65 & 120.65 & -0.166 & 0.328 & -0.461 & 0.251 & -0.139 & 0.192 & -0.414 & 0.190 & 13 & D & 550.9 & $\mathrm{C}$ & 550.9 \\
\hline 188 & TCU091 & 24.98 & 121.26 & -0.154 & 0.269 & -0.099 & 0.243 & -0.062 & 0.140 & 0.055 & 0.176 & 8 & $\mathrm{C}$ & $* * * * *$ & $*$ & 353.8 \\
\hline
\end{tabular}


Table 1. (Continued)

\begin{tabular}{|c|c|c|c|c|c|c|c|c|c|c|c|c|c|c|c|c|}
\hline \multirow{3}{*}{$\begin{array}{l}\text { No. } \\
189\end{array}$} & \multirow{3}{*}{$\begin{array}{c}\text { Stname } \\
\text { TCU092 }\end{array}$} & \multirow{3}{*}{$\begin{array}{c}\begin{array}{c}\text { Lat } \\
\left({ }^{\circ} \mathbf{N}\right)\end{array} \\
25.10\end{array}$} & \multirow{3}{*}{$\begin{array}{c}\text { Long } \\
\left({ }^{\circ} \mathbf{E}\right)\end{array}$} & \multicolumn{4}{|c|}{ TotalRes } & \multicolumn{4}{|c|}{ IntraRes } & \multirow{3}{*}{$\begin{array}{c}\mathbf{R} \\
13\end{array}$} & \multirow{3}{*}{$\frac{\text { Site } \dagger}{D}$} & \multirow{3}{*}{$\begin{array}{c}\begin{array}{c}\text { Vs30 } \\
\left(\mathbf{m ~ s}^{-1}\right)\end{array} \\
* * * * *\end{array}$} & \multirow{3}{*}{$\frac{\text { Site }}{*}$} & \multirow{3}{*}{$\begin{array}{r}\begin{array}{r}\text { Vs30N } \\
\left(\mathbf{m ~ s}^{-1}\right)\end{array} \\
295.3\end{array}$} \\
\hline & & & & \multicolumn{2}{|c|}{ PGAh \pm sd } & \multicolumn{2}{|c|}{$P G V h \pm s d$} & PGA & $\pm \mathrm{sd}$ & PGV & $\pm \mathrm{sd}$ & & & & & \\
\hline & & & & 0.189 & 0.377 & 0.149 & 0.370 & 0.129 & 0.322 & 0.155 & 0.249 & & & & & \\
\hline 190 & TCU093 & 24.85 & 120.95 & 0.388 & 163 & 0.284 & 132 & 0.338 & 0.133 & 0.286 & .108 & 5 & D & $* * * * *$ & * & 233.2 \\
\hline 191 & TCU094 & 24.77 & 121.05 & -0.150 & 0.178 & -0.025 & 148 & -0.184 & 0.120 & -0.020 & 0.129 & 15 & $\mathrm{C}$ & 581.5 & $\mathrm{C}$ & 581.5 \\
\hline 192 & TCU095 & 24.69 & 121.01 & 0.419 & 0.500 & 0.070 & 344 & 0.391 & 0.371 & 0.047 & 0.312 & 18 & $\mathrm{C}$ & 454.1 & $\mathrm{C}$ & 454.1 \\
\hline 193 & TCU096 & 24.79 & 120.96 & 0.180 & 0.371 & 0.333 & 0.186 & 0.111 & 0.256 & 0.238 & 0.187 & 12 & $\mathrm{D}$ & 456.9 & $\mathrm{C}$ & 456.9 \\
\hline 194 & TCU097 & 24.79 & 120.92 & 0.173 & 0.214 & 0.025 & 0.153 & 0.124 & 0.256 & -0.061 & 0.208 & 5 & D & $* * * * *$ & $*$ & 436.4 \\
\hline 195 & TCU098 & 24.74 & 120.90 & 0.353 & 0.363 & 0.154 & 0.173 & 0.373 & 0.204 & 0.152 & 0.169 & 21 & D & $* * * * *$ & $*$ & 297.2 \\
\hline 196 & TCU099 & 24.14 & 120.69 & 0.004 & 0.110 & 0.165 & 0.131 & -0.093 & 0.010 & 0.101 & 0.095 & 5 & D & $* * * *$ & $*$ & 325.6 \\
\hline 197 & TCU100 & 24.19 & 120.62 & -0.390 & 0.481 & -0.137 & 0.146 & -0.266 & 0.191 & -0.162 & 0.136 & 11 & $\mathrm{C}$ & $* * * * *$ & $*$ & 522.9 \\
\hline 198 & TCU101 & 24.24 & 120.71 & -0.063 & 0.238 & -0.272 & 0.059 & -0.123 & 0.138 & -0.270 & 0.117 & 9 & D & $* * * *$ & $*$ & 635.3 \\
\hline 199 & TCU102 & 24.25 & 120.72 & -0.385 & 0.392 & -0.106 & 0.160 & -0.386 & 0.172 & -0.132 & 0.147 & 18 & D & 706.2 & $\mathrm{C}$ & 706.2 \\
\hline 200 & TCU103 & 24.31 & 120.72 & -0.369 & 0.535 & -0.188 & 0.158 & -0.352 & 0.187 & -0.178 & 0.137 & 13 & $\mathrm{C}$ & 488.7 & $\mathrm{C}$ & 488.7 \\
\hline 201 & TCU104 & 24.21 & 120.60 & -0.235 & 0.558 & -0.155 & 0.219 & -0.233 & 0.243 & -0.172 & 0.260 & 17 & $\mathrm{C}$ & 403.5 & $\mathrm{C}$ & 403.5 \\
\hline 202 & TCU105 & 24.24 & 120.56 & -0.080 & 0.496 & 0.043 & 0.133 & -0.052 & 0.268 & 0.018 & 0.194 & 15 & $\mathrm{C}$ & 539.1 & $\mathrm{C}$ & 539.1 \\
\hline 203 & TCU106 & 24.09 & 120.55 & 0.228 & 0.576 & 0.228 & 0.259 & 0.311 & 0.261 & 0.298 & 0.136 & 18 & D & 451.0 & $\mathrm{C}$ & 451.0 \\
\hline 204 & TCU107 & 24.07 & 120.54 & 0.278 & 0.388 & 0.491 & 90 & 0 & 0 . & 25 & 13 & 24 & D & $* * * * *$ & $*$ & 151.9 \\
\hline 205 & TCU108 & 24.09 & 120.52 & 0.326 & 0.489 & 0.372 & 0.206 & 0.349 & 0.273 & 0.379 & 0.157 & 11 & $\mathrm{E}$ & $* * * * *$ & * & 197.2 \\
\hline 206 & TCU109 & 24.08 & 120.57 & 0.317 & 0.439 & 0.307 & 0.212 & & 0.220 & & 96 & 22 & D & $* * * * *$ & * & 212.9 \\
\hline 207 & TCU110 & 23.96 & 120.57 & 0.271 & 0.161 & 0.503 & 0.171 & 0.299 & 0.105 & 0.521 & 0.151 & 13 & E & 199.5 & $\mathrm{D}$ & 199.5 \\
\hline 208 & TCU111 & 24.11 & 120.49 & 0 . & 0.494 & 0.509 & 0.150 & 0.207 & 0.221 & 0.532 & 0.148 & 17 & $\mathrm{E}$ & 233.5 & D & 233.5 \\
\hline 209 & TCU112 & 24.06 & 120.42 & 0. & 0.620 & 0.447 & 0.254 & 0 & 0.283 & & 78 & 20 & $\mathrm{E}$ & 191.3 & D & 191.3 \\
\hline 210 & U113 & 23.89 & 120.39 & 94 & & 0.342 & 0.404 & 0. & 0.2 & 0.368 & 0.314 & 23 & E & 231.1 & D & 231.1 \\
\hline 211 & TCU114 & 23.88 & 120.52 & 0.292 & 0.588 & 0.294 & 0.666 & 0.374 & 0.398 & 0.390 & 0.364 & 6 & $\mathrm{E}$ & 264.6 & $\mathrm{D}$ & 264.6 \\
\hline 212 & TCU115 & 23.96 & 120.47 & & 0.304 & 0.457 & & & & & 38 & 21 & $\mathrm{E}$ & 84.9 & D & 184.9 \\
\hline 213 & TCU116 & 23.86 & 120.58 & 0.100 & 0.521 & 0.1 & 0.369 & & & & & 19 & E & 463.3 & $\mathrm{C}$ & 463.3 \\
\hline 214 & TCU117 & 24.13 & 120.46 & 0.362 & 0.518 & 0.532 & 0.275 & 0.405 & 0.246 & 0.574 & 0.222 & 24 & $\mathrm{E}$ & 207.2 & $\mathrm{D}$ & 207.2 \\
\hline 215 & TCU118 & 24.00 & 120.42 & & 0.438 & 0.426 & & & & & 17 & 23 & $\mathrm{E}$ & 28.6 & D & 228.6 \\
\hline 216 & TCU119 & 23.92 & 120.31 & 0.376 & 0.521 & 0.332 & 0.389 & 0.364 & 0.357 & 0.329 & 0.316 & 19 & $\mathrm{E}$ & $* * * * *$ & $*$ & 216.1 \\
\hline 217 & TCU120 & 23.98 & 120.61 & 0.024 & 0.348 & 0.225 & 0.181 & 0.082 & 0.184 & 0.252 & 0.273 & 13 & $\mathrm{C}$ & 458.2 & $\mathrm{C}$ & 458.2 \\
\hline 218 & TCU121 & 23.90 & 120.45 & & & & & & & & & 10 & $\mathrm{E}$ & 232.4 & $\mathrm{D}$ & 232.4 \\
\hline 219 & TCU122 & 23.81 & 120.61 & 0 & 0 & 0.056 & 39 & 0.2 & 0.3 & 0. & 0.269 & 17 & D & ****** & $*$ & 345.2 \\
\hline 220 & TCU123 & 24.02 & 120.54 & 0.332 & 0.284 & 0.555 & 0.169 & 0.391 & 0.091 & 0.589 & 0.141 & 17 & D & $* * * * *$ & * & 135.2 \\
\hline 221 & TCU124 & 23.91 & 120.68 & & 0.418 & 0.044 & 0.471 & 0.234 & 0.3 & 0.093 & 0.378 & 7 & D & $* * * * *$ & $*$ & 330.6 \\
\hline 222 & TCU125 & 23.96 & 120.68 & 0.094 & 0.194 & 0.115 & 0.227 & 0.126 & 0.165 & 0.150 & 0.140 & 11 & D & $* * * * *$ & $*$ & 298.5 \\
\hline 223 & TCU126 & 23.76 & 120.68 & 0.184 & 0.528 & 0.346 & 0.389 & 0.402 & 0.272 & 0.368 & 0.352 & 4 & D & $* * * * *$ & $*$ & 201.2 \\
\hline 224 & TCU127 & 24.63 & 121.00 & -0.123 & 0.360 & -0.313 & 0.299 & -0.254 & 0.369 & -0.367 & & 8 & D & 807.7 & B & 807.7 \\
\hline 225 & TCU128 & 24.42 & 120.76 & -0.253 & 0.585 & -0.038 & 0.251 & -0.227 & 0.294 & -0.053 & & 14 & $\mathrm{C}$ & 592.0 & $\mathrm{C}$ & 592.0 \\
\hline 226 & TCU129 & 23.88 & 120.68 & 0.935 & 0.734 & 0.263 & 0.388 & 0.949 & 0.654 & 0.257 & 0.367 & 20 & D & 506.5 & $\mathrm{C}$ & 506.5 \\
\hline 227 & TCU130 & 24.15 & 121.26 & -0.305 & 0.174 & -0.463 & 0.106 & & 0.223 & -0.342 & & 6 & B & $* * * * *$ & $*$ & 723.2 \\
\hline 228 & TCU131 & 24.57 & 120.82 & -0.002 & 0.280 & 0.014 & 0.170 & -0.057 & 0.194 & 0.005 & 0.160 & 16 & D & 491.4 & $\mathrm{C}$ & 491.4 \\
\hline 229 & TCU132 & 24.58 & 120.82 & -0.032 & 0.221 & 0.047 & 0.213 & -0.010 & 0.159 & 0.229 & 0.089 & 3 & D & $* * * * *$ & $*$ & 258.8 \\
\hline 230 & TCU133 & 24.14 & 120.65 & & & 0.372 & & 0.625 & & & 0.301 & 6 & D & $* * * * *$ & $*$ & 154.0 \\
\hline 231 & TCU134 & 24.12 & 120.62 & 0.302 & 0.078 & 0.425 & 0.011 & 0.095 & 0.025 & 0.391 & 0.011 & 2 & D & $* * * * *$ & $*$ & 193.2 \\
\hline 232 & TCU135 & 24.33 & 120.65 & 0.061 & 0.019 & 0.432 & 0.215 & -0.153 & 0.029 & 0.280 & 0.090 & 3 & $\mathrm{C}$ & 619.8 & $\mathrm{C}$ & 619.8 \\
\hline 233 & TCU136 & 24.26 & 120.65 & -0.215 & 0.495 & -0.129 & 0.186 & -0.199 & 0.16 & -0.133 & 0.144 & 13 & $\mathrm{C}$ & 458.0 & $\mathrm{C}$ & 458.0 \\
\hline 234 & TCU137 & 24.19 & 120.92 & -0.351 & 0.592 & -0.812 & 0.478 & -0.069 & 1.236 & -0.588 & 0.609 & 6 & B & $* * * * *$ & $*$ & 1126.7 \\
\hline 235 & TCU138 & 23.92 & 120.60 & & 0.263 & 0.316 & 0.111 & & 0.130 & 0.331 & 0.070 & 9 & D & 604.2 & $\mathrm{C}$ & 604.2 \\
\hline 236 & TCU139 & 23.92 & 120.54 & & 0.071 & 0.866 & 0.019 & 0.104 & 0.037 & 0.566 & 0.002 & 2 & $\mathrm{E}$ & 307.0 & $\mathrm{D}$ & 307.0 \\
\hline 237 & TCU140 & 23.96 & 120.36 & 0.491 & 0.585 & 0.606 & 0.257 & 0.469 & 0.214 & 0.570 & 0.151 & 13 & $\mathrm{E}$ & 221.9 & D & 221.9 \\
\hline 238 & TCU141 & 23.83 & 120.46 & 0.343 & 0.492 & 0.621 & 0.238 & 0.344 & 0.250 & 0.545 & 0.201 & 13 & E & 221.2 & D & 221.2 \\
\hline 239 & TCU143 & 23.88 & 120.76 & & 0.096 & -0.322 & 0.070 & 0.044 & 0.005 & -0.140 & 0.007 & 3 & D & 465.9 & $\mathrm{C}$ & 465.9 \\
\hline 240 & TCU144 & 24.09 & 120.44 & 0.965 & 0.564 & 0.984 & 0.087 & 0.687 & 0.493 & 0.772 & 0.224 & 2 & $\mathrm{E}$ & $* * * * *$ & $*$ & 97.2 \\
\hline 241 & TCU145 & 23.94 & 120.34 & 0.660 & 0.526 & 0.586 & 0.274 & 0.637 & 0.179 & 0.552 & 0.159 & 13 & $\mathrm{E}$ & $* * * * *$ & $*$ & 144.6 \\
\hline
\end{tabular}


Table 1. (Continued)

\begin{tabular}{|c|c|c|c|c|c|c|c|c|c|c|c|c|c|c|c|c|}
\hline \multirow{3}{*}{$\frac{\text { No. }}{242}$} & \multirow{3}{*}{$\begin{array}{c}\text { Stname } \\
\text { TCU146 }\end{array}$} & \multirow{3}{*}{$\begin{array}{c}\begin{array}{c}\text { Lat } \\
\left({ }^{\circ} \mathbf{N}\right)\end{array} \\
24.16\end{array}$} & \multirow{3}{*}{$\begin{array}{c}\text { Long } \\
\left({ }^{\circ} \mathbf{E}\right)\end{array}$} & \multicolumn{4}{|c|}{ TotalRes } & \multicolumn{4}{|c|}{ IntraRes } & \multirow{3}{*}{$\frac{\mathbf{R}}{4}$} & \multirow{3}{*}{$\frac{\text { Site } \dagger}{E}$} & \multirow{3}{*}{$\begin{array}{c}\text { Vs30 } \\
\left(\mathbf{m ~ s}^{-1}\right) \\
* * * * *\end{array}$} & \multirow{3}{*}{\begin{tabular}{|c|} 
Site $:$ \\
$*$
\end{tabular}} & \multirow{3}{*}{$\begin{array}{r}\begin{array}{r}\text { Vs30N } \\
\left(\mathbf{m ~ s}^{-1}\right)\end{array} \\
109.2\end{array}$} \\
\hline & & & & \multicolumn{2}{|c|}{ PGAh \pm sd } & \multicolumn{2}{|c|}{$P G V h \pm s d$} & PGA & $\pm \mathrm{sd}$ & PGV & $\pm \mathrm{sd}$ & & & & & \\
\hline & & & & 0.726 & 0.606 & 0.625 & 0.358 & 0.675 & 0.236 & 0.708 & 0.090 & & & & & \\
\hline 243 & CHY001 & 23.71 & 120.24 & 0.713 & .148 & 0.304 & 0.174 & 0.628 & 0.091 & 0.302 & 0.126 & 10 & $\mathrm{E}$ & 229.9 & D & 229.9 \\
\hline 244 & CHY002 & 23.72 & 120.41 & 0.381 & 0.418 & 0.522 & 0.337 & 0.365 & 0.207 & 0.492 & 0.236 & 16 & E & 229.6 & $\mathrm{D}$ & 229.6 \\
\hline 245 & CHY003 & 23.72 & 120.53 & 0.591 & 0.205 & 0.552 & 0.226 & 0.437 & 0.125 & 0.474 & 0.156 & 16 & D & 177.6 & E & 177.6 \\
\hline 246 & CHY004 & 23.60 & 120.17 & 0.468 & 0.317 & 0.284 & 0.155 & 0.441 & 0.246 & 0.273 & 0.129 & 26 & $\mathrm{E}$ & 273.0 & D & 273.0 \\
\hline 247 & CHY005 & 23.61 & 120.41 & 0.279 & 0.377 & 0.346 & 0.284 & 0.318 & 0.248 & 0.392 & 0.221 & 19 & $\mathrm{E}$ & 211.0 & $\mathrm{D}$ & 211.0 \\
\hline 248 & CHY006 & 23.58 & 120.55 & 0.277 & 0.277 & 0.327 & 0.308 & 0.312 & 0.144 & 0.359 & 0.197 & 25 & D & 422.7 & $\mathrm{C}$ & 422.7 \\
\hline 249 & CHY007 & 23.47 & 120.17 & 0.350 & 0.183 & & & & & & & 11 & E & 202.1 & D & 202.1 \\
\hline 250 & CHY008 & 23.49 & 120.27 & 0.563 & 0.358 & 0.478 & 0.342 & 0.517 & 0.192 & 0.474 & 0.234 & 27 & $\mathrm{E}$ & 209.5 & D & 209.5 \\
\hline 251 & CHY009 & 23.47 & 120.41 & 0.517 & 0.300 & 0.500 & 0.422 & 0.506 & 0.215 & 0.471 & 0.333 & 26 & D & 226.1 & D & 226.1 \\
\hline 252 & CHY010 & 23.47 & 120.54 & 0.260 & 0.487 & 0.074 & 0.367 & 0.255 & 0.366 & 0.056 & 0.266 & 24 & D & $*$ & $*$ & 353.0 \\
\hline 253 & CHY011 & 23.48 & 120.65 & 0.278 & 0.569 & 0.033 & 0.763 & 0.207 & 0.638 & 0.001 & 0.738 & 8 & B & $* * * * *$ & $*$ & 390.1 \\
\hline 254 & CHY012 & 23.33 & 120.15 & 0.394 & 0.142 & 0.359 & 0.253 & 0.379 & 0.086 & 0.369 & 0.142 & 21 & $\mathrm{E}$ & 192.2 & D & 192.2 \\
\hline 255 & CHY013 & 23.36 & 120.27 & 0.693 & 0.185 & 0.622 & 0.354 & 0.546 & 0.113 & 0.564 & 0.238 & 15 & $\mathrm{E}$ & 216.2 & $\mathrm{D}$ & 216.2 \\
\hline 256 & CHY014 & 23.30 & 120.58 & & 0.346 & 0.223 & 0.392 & 0.319 & 0.241 & & & 26 & D & 341.8 & D & 341.8 \\
\hline 257 & CHY015 & 23.36 & 120.41 & 0.640 & 0.233 & 0.628 & 0.293 & 0.584 & 0.161 & 0 & 20 & 28 & D & 24.7 & D & 224.7 \\
\hline 258 & CHY016 & 23.22 & 120.15 & 0.699 & 0.327 & 0.715 & 0.285 & 0.609 & 0.175 & 0.661 & 0.174 & 21 & E & 199.5 & D & 199.5 \\
\hline 259 & CHY017 & 23.21 & 120.27 & 0.314 & 0.340 & 0.371 & 0.251 & 0.244 & 0.169 & & 0.168 & 26 & $\mathrm{E}$ & 196.0 & D & 196.0 \\
\hline 260 & $\mathrm{CHY}$ & 23.22 & & & & & & & & & & 12 & $\mathrm{C}$ & & $\mathrm{C}$ & \\
\hline 261 & CHY019 & 23.18 & 120.48 & 0.477 & 0.543 & 0.049 & 0.438 & 0.406 & 0.376 & 0.008 & 0.344 & 16 & D & $* * * * *$ & $*$ & 384.8 \\
\hline 262 & CHY020 & 23.10 & 120.15 & 0.529 & 0.303 & 0.435 & 0.281 & 0.364 & 0.256 & 0.310 & 0. & 13 & E & 214.1 & D & 214.1 \\
\hline 263 & CHY021 & & & & & & & & & & & 14 & D & & $\mathrm{D}$ & \\
\hline 264 & CHY022 & 23.05 & 120.46 & 0.159 & 0.295 & -0.215 & 0.262 & 0.105 & 0.119 & -0.234 & 0.218 & 19 & $\mathrm{C}$ & 570.0 & $\mathrm{C}$ & 570.0 \\
\hline 265 & CHY023 & 22.97 & 120.28 & 0.472 & 0.232 & 0.405 & 0.207 & 0.423 & 0.154 & & 0. & 18 & D & 279.8 & D & 279.8 \\
\hline 266 & CHY024 & 23.76 & & & & & & & & & & 29 & D & 408.5 & $\mathrm{C}$ & \\
\hline 267 & CHY025 & 23.78 & 120.51 & 0.296 & 0.426 & 0.273 & 0.246 & 0.321 & 0.234 & 0.295 & 0.172 & 28 & $\mathrm{E}$ & 276.5 & D & 276.5 \\
\hline 268 & CHY026 & 23.80 & 120.41 & 0.058 & 0.473 & 0.143 & 0.310 & 0.064 & 0.199 & 0.149 & 0.267 & 28 & $\mathrm{E}$ & 220.6 & D & 220.6 \\
\hline 269 & CHY027 & 23.75 & 120.25 & 0.201 & 0.536 & 0.073 & 0.382 & 0.243 & 0.312 & 0.104 & & 24 & $\mathrm{E}$ & 209.5 & D & 209.5 \\
\hline 270 & CHY028 & 23.63 & 120.61 & 0.498 & 0.876 & 0.183 & 0.713 & 0.592 & 0.630 & 0.248 & 0.518 & 24 & D & 546.9 & $\mathrm{C}$ & 546.9 \\
\hline 271 & $\mathrm{CHYC}$ & 23.61 & & & & & & & & & & 27 & $\mathrm{C}$ & & $\mathrm{C}$ & \\
\hline 272 & CHY030 & 23.64 & 120.48 & & 0.387 & 0.548 & 0.350 & 0.476 & 0.295 & & & 21 & $\mathrm{E}$ & 207.9 & D & 207.9 \\
\hline 273 & CHY031 & 23.66 & 120.34 & 0.273 & 0.341 & 0.249 & 0.184 & 0.206 & 0.281 & 0.232 & 0.207 & 19 & $\mathrm{E}$ & 215.7 & D & 215.7 \\
\hline 274 & & 23.58 & & & & & & & & & & 27 & E & & D & 2.6 \\
\hline 275 & CHY033 & 23.54 & 120.22 & & 0.274 & 0.260 & 0.216 & 0.223 & 0.180 & & & 31 & $\mathrm{E}$ & 193.3 & D & 193.3 \\
\hline 276 & CHY034 & 23.52 & 120.54 & 0.360 & 0.161 & 0.369 & 0.322 & 0.335 & 0.073 & 0.346 & 0.187 & 19 & D & 383.1 & $\mathrm{C}$ & 383.1 \\
\hline 277 & & & & & & & & & & & & 30 & D & & $*$ & 9.1 \\
\hline 278 & CHY036 & 23.61 & 120.48 & 0.540 & & 0.499 & 0.439 & 0.607 & 35 & 0.549 & 0.320 & 23 & D & 236.1 & D & 236.1 \\
\hline 279 & CHY037 & 23.56 & 120.42 & 0.322 & 0.293 & 0.299 & 0.431 & 0.255 & 0.215 & 0.269 & 0.324 & 20 & D & 208.8 & D & 208.8 \\
\hline 280 & CHY038 & 23.54 & 120.45 & & & & & 0.717 & & & & 8 & $\mathrm{C}$ & 372.9 & $\mathrm{C}$ & 372.9 \\
\hline 281 & CHY039 & 23.52 & 120.34 & 0.205 & 0.308 & 0.359 & 0.295 & 0.241 & 0.168 & 0.378 & 0.202 & 33 & $\mathrm{E}$ & 195.2 & D & 195.2 \\
\hline 282 & CHY040 & 23.43 & 120.63 & -0.347 & 0.469 & -0.441 & 0.370 & -0.386 & 0.320 & -0.438 & 0.273 & 15 & B & $* * * * *$ & $*$ & 859.4 \\
\hline 283 & CHY041 & 23.44 & 120.60 & & 0.454 & & 0.381 & 0.347 & 0.294 & 0.159 & 0.248 & 25 & D & 488.1 & $\mathrm{C}$ & 488.1 \\
\hline 284 & CHY042 & 23.36 & 120.58 & -0.206 & 0.420 & -0.201 & 0.372 & -0.249 & 0.316 & -0.213 & 0.296 & 25 & B & $* * * * *$ & $*$ & 573.3 \\
\hline 285 & CHY043 & 23.41 & & & & & & & & & & 21 & $\mathrm{E}$ & 225.4 & D & 225.4 \\
\hline 286 & CHY044 & 23.38 & 120.16 & & & & 0.320 & 0.242 & 0.231 & & 0.260 & 29 & $\mathrm{E}$ & 192.7 & D & 192.7 \\
\hline 287 & CHY045 & 23.30 & 120.66 & -0.198 & 0.378 & -0.330 & 0.249 & -0.195 & 0.282 & -0.319 & 0.239 & 14 & B & 627.6 & $\mathrm{C}$ & 627.6 \\
\hline 288 & CHY046 & 23.48 & & & & & & & & & & 36 & $\mathrm{C}$ & 446.5 & $\mathrm{C}$ & 446.5 \\
\hline 289 & CHY047 & 23.49 & 120.45 & 0.764 & 0.520 & 0.623 & 0.305 & 0.809 & 0.252 & 0.618 & 0.185 & 19 & D & 183.5 & E & 183.5 \\
\hline 290 & CHY048 & 23.47 & 120.44 & 0.395 & 0.431 & 0.180 & 0.345 & 0.476 & 0.383 & 0.242 & 0.287 & 12 & D & 248.7 & D & 248.7 \\
\hline 291 & CHY049 & 23.37 & 120.36 & 0.450 & 0.347 & 0.504 & 0.316 & 0.412 & 0.213 & 0.478 & 0.242 & 33 & E & 224.8 & D & 224.8 \\
\hline 292 & CHY050 & 23.28 & 120.41 & 0.348 & 0.545 & 0.047 & 0.554 & 0.308 & 0.386 & 0.023 & 0.424 & 28 & $\mathrm{C}$ & $* * * * *$ & $*$ & 375.0 \\
\hline 293 & CHY051 & 23.28 & 120.46 & 0.522 & 0.072 & 0.335 & 0.029 & 0.467 & 0.079 & 0.266 & 0.064 & 4 & D & 363.9 & $\mathrm{C}$ & 363.9 \\
\hline 294 & CHY052 & 23.29 & 120.50 & 0.127 & 0.287 & -0.057 & 0.403 & 0.087 & 0.142 & -0.089 & 0.245 & 20 & $\mathrm{~B}$ & $* * * * *$ & $*$ & 458.9 \\
\hline
\end{tabular}


Table 1. (Continued)

\begin{tabular}{|c|c|c|c|c|c|c|c|c|c|c|c|c|c|c|c|c|}
\hline \multirow{3}{*}{$\begin{array}{l}\text { No. } \\
295\end{array}$} & \multirow{3}{*}{$\begin{array}{c}\text { Stname } \\
\text { CHY053 }\end{array}$} & \multirow{3}{*}{$\begin{array}{c}\begin{array}{c}\text { Lat } \\
\left({ }^{\circ} \mathbf{N}\right)\end{array} \\
23.29\end{array}$} & \multirow{3}{*}{$\begin{array}{c}\text { Long } \\
\left({ }^{\circ} \mathbf{E}\right)\end{array}$} & \multicolumn{4}{|c|}{ TotalRes } & \multicolumn{4}{|c|}{ IntraRes } & \multirow{3}{*}{$\begin{array}{l}\mathbf{R} \\
12\end{array}$} & \multirow{3}{*}{$\frac{\text { Site } \dagger}{D}$} & \multirow{3}{*}{$\begin{array}{c}\begin{array}{c}\text { Vs30 } \\
\left(\mathbf{m ~ s}^{-1}\right)\end{array} \\
272.7\end{array}$} & \multirow{3}{*}{$\frac{\text { Site } F}{D}$} & \multirow{3}{*}{$\begin{array}{r}\begin{array}{r}\text { Vs30N } \\
\left(\mathbf{m ~ s}^{-1}\right)\end{array} \\
272.7\end{array}$} \\
\hline & & & & \multicolumn{2}{|c|}{ PGAh \pm sd } & \multicolumn{2}{|c|}{$P G V h \pm s d$} & PGA & $\pm \mathrm{sd}$ & PGV & $\pm \mathrm{sd}$ & & & & & \\
\hline & & & & 0.875 & 0.310 & 0.904 & 0.487 & 0.733 & 0.289 & 0.791 & 0.418 & & & & & \\
\hline 296 & CHY054 & 23.31 & 120.31 & 0.452 & 382 & 0.577 & 309 & 0.444 & 0.223 & 0.579 & 0.197 & 32 & E & 175.1 & E & 175.1 \\
\hline 297 & CHY055 & 23.27 & 120.27 & 0.616 & 0.490 & 0.652 & 346 & 0.568 & 0.297 & 0.590 & 0.249 & 21 & $\mathrm{E}$ & 221.5 & $\mathrm{D}$ & 221.5 \\
\hline 298 & CHY056 & 23.26 & 120.13 & 0.362 & 0.269 & 0.376 & 0.319 & 0.329 & 0.189 & 0.342 & 0.270 & 21 & $\mathrm{E}$ & 189.0 & D & 189.0 \\
\hline 299 & CHY057 & 23.15 & 120.41 & 0.257 & 0.354 & 0.022 & 0.358 & 0.177 & 0.239 & -0.033 & 0.312 & 24 & $\mathrm{C}$ & $* * * * *$ & $*$ & 414.5 \\
\hline 300 & CHY058 & 23.17 & 120.32 & 0.304 & 0.285 & 0.410 & 0.241 & 0.322 & 0.179 & 0.443 & 0.211 & 22 & D & 271.1 & D & 271.1 \\
\hline 301 & CHY059 & 23.18 & 120.10 & 0.191 & 0.144 & 0.262 & 0.288 & 0.163 & 0.094 & 0.248 & 0.220 & 20 & $\mathrm{E}$ & 187.0 & D & 187.0 \\
\hline 302 & CHY060 & 23.12 & 120.24 & 0.217 & 0.311 & 0.286 & 0.224 & 0.133 & 0.257 & 0.253 & 0.214 & 24 & $\mathrm{E}$ & 223.9 & $\mathrm{D}$ & 223.9 \\
\hline 303 & CHY061 & 23.08 & 120.51 & -0.180 & 0.201 & -0.435 & 0.216 & -0.252 & 0.108 & -0.457 & 0.148 & 21 & D & was & $*$ & 890.2 \\
\hline 304 & CHY062 & 23.12 & 120.45 & -0.099 & 0.234 & -0.314 & 0.224 & -0.143 & 0.154 & -0.334 & .169 & 29 & D & 597.9 & $\mathrm{C}$ & 597.9 \\
\hline 305 & CHY063 & 23.03 & 120.34 & 0.005 & 0.162 & 0.117 & 0.174 & -0.006 & 0.136 & 0.107 & 0.126 & 25 & D & 287.7 & D & 287.7 \\
\hline 306 & CHY064 & 23.00 & 120.23 & 0.958 & 0.000 & 0.529 & 0.000 & 0.627 & 0.000 & 0.526 & 0.000 & 1 & D & $* * * * *$ & $*$ & 151.4 \\
\hline 307 & CHY065 & 22.91 & 120.34 & 0.260 & 0.256 & 0.436 & 0.198 & 0.226 & 0.210 & 0.433 & 0.126 & 17 & D & 222.7 & D & 222.7 \\
\hline 308 & CHY066 & 22.92 & 120.21 & 0.039 & 0.127 & 0.479 & 0.234 & 0.007 & 0.099 & 0.476 & 0.171 & 17 & $\mathrm{E}$ & 211.5 & D & 211.5 \\
\hline 309 & CHY067 & 23.00 & 120.18 & 0.528 & 0.401 & 0.587 & 0.388 & 0.464 & 0.260 & & 90 & 14 & $\mathrm{E}$ & 229.0 & D & 229.0 \\
\hline 310 & CHY068 & 22.99 & 120.20 & 0.234 & 0.208 & 0.209 & 26 & 36 & 0.092 & 0 & 48 & 5 & D & 96.1 & $\mathrm{D}$ & 196.1 \\
\hline 311 & CHY069 & 22.97 & 120.18 & 0.220 & 0.231 & 0.383 & 0.213 & 0.189 & 0.117 & 0.371 & 0.095 & 13 & E & 220.0 & D & 220.0 \\
\hline 312 & CHY070 & 22.97 & 120.23 & -0.033 & 0.124 & 0.144 & 0.146 & -0.062 & 0.082 & & 0.086 & 20 & D & 228.7 & $\mathrm{D}$ & 228.7 \\
\hline 313 & CHY071 & 23.07 & 120.16 & 0.294 & 0.175 & 0.517 & 0.215 & 0.237 & 0.165 & 0.456 & 0.213 & 21 & E & 200.5 & $\mathrm{D}$ & 200.5 \\
\hline 314 & CHY072 & 23.07 & 120.12 & 0.111 & 0.390 & 0.105 & 0.237 & 0.011 & 0.404 & 0.137 & 0.281 & 6 & $\mathrm{E}$ & $* * * * *$ & $*$ & 305.1 \\
\hline 315 & CHY073 & 23.50 & 120.42 & 0.189 & 0.440 & 0.285 & 0.494 & 0 & & 0.2 & 0.405 & 34 & D & 201.5 & D & 201.5 \\
\hline 316 & CHY074 & 23.51 & 120.81 & -0.214 & 0.350 & -0.103 & 0.387 & -0.123 & 0.177 & -0.059 & 0.235 & 19 & B & 524.6 & $\mathrm{C}$ & 524.6 \\
\hline 317 & CHY075 & 23.57 & 119.56 & 0.401 & 0.240 & -0.143 & 0.052 & 0.502 & 0.095 & -0.131 & 0.091 & 11 & B & 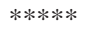 & $*$ & 494.4 \\
\hline 318 & CHY076 & 23.64 & 120.22 & 0.481 & .459 & & & & & & & 33 & $\mathrm{E}$ & 70.9 & E & 170.9 \\
\hline 319 & CHY077 & 23.18 & 120.19 & & 0.673 & & & & & & & 19 & E & 124.9 & E & 124.9 \\
\hline 320 & CHY078 & 23.04 & 120.23 & 0.231 & 0.241 & 0.347 & 0.165 & 0.199 & 0.125 & 0.317 & 0.112 & 17 & D & 162.3 & $\mathrm{E}$ & 162.3 \\
\hline 321 & CHY079 & 23.18 & 120.53 & -0.268 & 0.365 & -0.466 & 0.298 & -0.314 & 0.232 & -0.485 & 0.195 & 18 & $\mathrm{C}$ & & $*$ & 936.2 \\
\hline 322 & CHY080 & 23.60 & 120.68 & -0.143 & 0.444 & -0.034 & 0.476 & 0.047 & 0.412 & 0.072 & 0.415 & 15 & B & 499.2 & $\mathrm{C}$ & 499.2 \\
\hline 323 & CHY081 & 23.27 & 120.49 & -0.532 & 0.351 & -0.281 & 0.438 & -0.551 & 0.255 & -0.300 & 0.324 & 25 & $\mathrm{C}$ & $* * * *$ & $*$ & 670.5 \\
\hline 324 & CHY082 & 23.72 & 120.30 & & & & & & & & & 27 & $\mathrm{E}$ & 4.8 & $\mathrm{D}$ & 194.8 \\
\hline 325 & CHY083 & 23.72 & 120.58 & & 0 & 1 & 10 & & 0.202 & & & 14 & D & 374.0 & $\mathrm{C}$ & 374.0 \\
\hline 326 & CHY084 & 23.73 & 120.46 & 0.140 & 0.302 & 0.171 & 0.208 & 0.157 & 0.179 & 0.199 & 0.139 & 11 & $\mathrm{E}$ & 259.1 & $\mathrm{D}$ & 259.1 \\
\hline 327 & CHY086 & 23.35 & 120.59 & & 0.447 & 0.2 & 0.420 & 0.035 & 0.3 & 0.207 & 0.293 & 18 & B & ( & $*$ & 269.3 \\
\hline 328 & CHY087 & 23.38 & 120.52 & 0.156 & 0.436 & 0.102 & 0.334 & 0.143 & 0.268 & 0.077 & 0.269 & 30 & $\mathrm{C}$ & 508.0 & $\mathrm{C}$ & 508.0 \\
\hline 329 & CHY088 & 23.35 & 120.43 & 0.588 & 0.403 & 0.495 & 0.310 & 0.641 & 0.176 & 0.545 & 0.215 & 30 & D & $* * * * *$ & $*$ & 146.5 \\
\hline 330 & CHY089 & 23.08 & 120.36 & 0.066 & 0.000 & 0.048 & 0.000 & & 0.000 & & 0.000 & 1 & $\mathrm{C}$ & 396.2 & $\mathrm{C}$ & 396.2 \\
\hline 331 & CHY090 & 23.27 & 120.22 & 0.646 & 0.496 & 0.695 & 0.346 & 0.634 & 0.296 & & 0.269 & 28 & $\mathrm{E}$ & 181.9 & D & 181.9 \\
\hline 332 & CHY091 & 23.08 & 120.21 & 0.171 & 0.220 & 0.259 & 0.274 & 0.123 & 0.188 & 0.282 & 0.274 & 9 & $\mathrm{E}$ & $* * * *$ & $*$ & 235.1 \\
\hline 333 & CHY092 & 23.79 & 120.48 & & & & & & 0.091 & & & 17 & $\mathrm{E}$ & 252.2 & D & 252.2 \\
\hline 334 & CHY093 & 23.65 & 120.15 & 0.395 & 0.436 & 0.265 & 0.189 & 0.360 & 0.232 & 0.231 & 0.185 & 18 & $\mathrm{E}$ & 191.4 & D & 191.4 \\
\hline 335 & CHY094 & 23.79 & 120.32 & 0.272 & 0.418 & 0.391 & 0.247 & 0.254 & 0.172 & 0.365 & 0.171 & 19 & E & 223.2 & D & 223.2 \\
\hline 336 & CHY095 & 23.45 & 120.32 & & & & & 0.478 & 0.103 & & & 25 & $\mathrm{E}$ & 216.4 & D & 216.4 \\
\hline 337 & CHY096 & 22.98 & 120.23 & -0.134 & 0.136 & 0.261 & 0.150 & -0.098 & 0.049 & 0.262 & 0.070 & 12 & D & $* * * * *$ & $*$ & 243.6 \\
\hline 338 & CHY097 & 23.01 & 120.20 & 0.174 & & & & & 0.290 & & & 6 & D & ** & $*$ & 360.7 \\
\hline 339 & CHY098 & 23.00 & 120.16 & & 0.148 & 0.317 & 0.211 & 010 & 0.03 & 0.253 & 0.240 & 5 & $\mathrm{E}$ & $* * * * *$ & $*$ & 247.5 \\
\hline 340 & CHY099 & 23.14 & 120.28 & 0.342 & 0.224 & 0.443 & 0.202 & 0.253 & 0.131 & 0.352 & 0.188 & 21 & D & 217.1 & D & 217.1 \\
\hline 341 & CHY100 & 23.23 & 120.34 & & 0.352 & 0.490 & 0.365 & 0.469 & 0.243 & 0.419 & 0.313 & 23 & D & $* * * * *$ & $*$ & 183.8 \\
\hline 342 & CHY101 & 23.69 & 120.56 & 0.289 & 0.485 & 0.410 & 0.411 & 0.419 & 0.230 & 0.469 & 0.234 & 21 & D & 252.4 & D & 252.4 \\
\hline 343 & CHY102 & 23.25 & 120.61 & -0.148 & 0.369 & -0.611 & 0.282 & -0.187 & 0.178 & -0.652 & 0.153 & 22 & B & 836.1 & B & 836.1 \\
\hline 344 & CHY103 & 23.70 & 120.53 & 0.149 & 0.508 & 0.505 & 0.105 & 0.279 & 0.312 & 0.478 & 0.143 & 4 & D & 223.7 & D & 223.7 \\
\hline 345 & CHY104 & 23.67 & 120.47 & & 0.314 & 0.669 & 0.297 & 0.497 & 0.144 & 0.681 & 0.174 & 16 & E & 218.8 & D & 218.8 \\
\hline 346 & CHY105 & 23.56 & 120.34 & 0.187 & 0.375 & 0.356 & 0.313 & 0.123 & 0.174 & 0.267 & 0.227 & 15 & $\mathrm{E}$ & 204.4 & D & 204.4 \\
\hline 347 & CHY106 & 23.44 & 120.41 & 0.503 & 0.355 & 0.573 & 0.446 & 0.470 & 0.232 & 0.544 & 0.327 & 22 & D & 227.6 & D & 227.6 \\
\hline
\end{tabular}


Table 1. (Continued)

\begin{tabular}{|c|c|c|c|c|c|c|c|c|c|c|c|c|c|c|c|c|}
\hline \multirow{3}{*}{$\begin{array}{l}\text { No. } \\
348\end{array}$} & \multirow{3}{*}{$\begin{array}{c}\text { Stname } \\
\text { CHY107 }\end{array}$} & \multirow{3}{*}{$\begin{array}{c}\begin{array}{c}\text { Lat } \\
\left({ }^{\circ} \mathbf{N}\right)\end{array} \\
23.30\end{array}$} & \multirow{3}{*}{$\begin{array}{c}\begin{array}{c}\text { Long } \\
\left({ }^{\circ} \mathbf{E}\right)\end{array} \\
120.29\end{array}$} & \multicolumn{4}{|c|}{ TotalRes } & \multicolumn{4}{|c|}{ IntraRes } & \multirow{3}{*}{$\begin{array}{l}\mathbf{R} \\
20\end{array}$} & \multirow{3}{*}{$\frac{\text { Site } \dagger}{E}$} & \multirow{3}{*}{$\begin{array}{c}\begin{array}{c}\text { Vs30 } \\
\left(\mathbf{m ~ s}^{-1}\right)\end{array} \\
181.1\end{array}$} & \multirow{3}{*}{$\frac{\text { Site }+}{D}$} & \multirow{3}{*}{$\begin{array}{r}\begin{array}{r}\text { Vs30N } \\
\left(\mathbf{m ~ s}^{-1}\right)\end{array} \\
181.1\end{array}$} \\
\hline & & & & \multicolumn{2}{|c|}{ PGAh \pm sd } & \multicolumn{2}{|c|}{ PGVh \pm sd } & PGAh & $\pm s d$ & PGV & $\pm s d$ & & & & & \\
\hline & & & & 0.554 & 0.265 & 0.549 & 0.368 & 0.489 & 0.188 & 0.494 & 0.278 & & & & & \\
\hline 349 & CHY108 & 23.19 & 120.25 & 0.998 & 0.721 & 1.030 & 0.117 & 0.932 & & 1.012 & & 3 & $\mathrm{E}$ & 249.7 & D & 249.7 \\
\hline 350 & CHY109 & 23.25 & 120.53 & -0.797 & 0.000 & -0.557 & 0.000 & -0.884 & 0.000 & -0.592 & 0.000 & 1 & B & $* * * * *$ & $*$ & 1135.3 \\
\hline 351 & CHY111 & 23.79 & 120.23 & 0.719 & 0.549 & 0.291 & 0.554 & 0.622 & 0.280 & 0.249 & 0.423 & 13 & E & 269.6 & D & 269.6 \\
\hline 352 & CHY112 & 23.70 & 120.18 & 0.695 & 0.774 & 0.249 & & 0.608 & & & 0.292 & 7 & $\mathrm{E}$ & 239.7 & D & 239.7 \\
\hline 353 & CHY113 & 23.42 & 120.21 & 0.494 & 0.465 & 0.546 & 0.276 & 0.391 & 0.207 & 0.450 & 0.249 & 5 & $\mathrm{E}$ & 240.6 & D & 240.6 \\
\hline 354 & CHY114 & 23.04 & 120.12 & 0.363 & 0.111 & 0.488 & 0.225 & 0.336 & 0.109 & 0.454 & 0.169 & 14 & E & 215.1 & D & 215.1 \\
\hline 355 & CHY115 & 23.15 & 120.10 & 0.468 & 0.192 & 0.449 & 0.139 & 0.419 & 0.072 & 0.436 & 0.075 & 12 & $\mathrm{E}$ & $* * * * *$ & $*$ & 178.0 \\
\hline 356 & CHY116 & 23.08 & 120.11 & 0.624 & 0.237 & 0.635 & 0.156 & 0.580 & 0.119 & 0.601 & 0.096 & 14 & E & 194.3 & $\mathrm{D}$ & 194.3 \\
\hline 357 & CHY117 & 23.80 & 120.22 & 0.280 & 0.643 & 0.106 & 0.320 & 0.233 & 0.275 & -0.005 & 0.318 & 4 & $\mathrm{E}$ & $* * * *$ & $*$ & 394.5 \\
\hline 358 & CHY123 & 23.48 & 120.24 & 0.415 & 0.000 & 0.380 & 0.000 & 0.088 & 0.000 & 0.347 & 0.000 & 1 & E & 212.5 & D & 212.5 \\
\hline 359 & KAU001 & 23.16 & 120.64 & -0.733 & 0.253 & -0.799 & 0.311 & -0.739 & 0.113 & -0.791 & 0.197 & 11 & D & 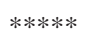 & $*$ & 1624.6 \\
\hline 360 & KAU002 & 22.62 & 120.31 & -0.230 & 0.029 & -0.303 & & & 0.008 & & & 2 & E & $* * * *$ & $*$ & 633.8 \\
\hline 361 & KAU003 & 22.63 & 120.26 & -0.220 & 0.227 & -0.212 & 0.141 & -0.244 & 0.124 & -0.242 & 0.122 & 14 & D & 887.7 & B & 887.7 \\
\hline 362 & KAU004 & 22.63 & 120.34 & -0.257 & 0.089 & -0.110 & 0.399 & -0.352 & 0.004 & 0.030 & 0.239 & 2 & E & 221.9 & D & 221.9 \\
\hline 363 & KAU005 & 22.62 & 120.34 & -0.164 & 0.020 & -0.076 & 0.017 & & 0.112 & -0.066 & & 3 & $\mathrm{E}$ & 3.5 & $\mathrm{D}$ & 3.5 \\
\hline 364 & KAU006 & 22.59 & 120.32 & -0.225 & 0.138 & 0.213 & 0.266 & -0.335 & 0.101 & 0.188 & 0.181 & 9 & $\mathrm{E}$ & 219.0 & D & 219.0 \\
\hline 365 & KAU007 & 22.65 & 120.36 & -0.151 & 0.091 & 0.096 & 130 & -0.170 & 0.048 & 0.097 & 0.100 & 16 & D & & $*$ & 327.8 \\
\hline 366 & KAU008 & 22.63 & 120.37 & -0.105 & 0.158 & 0.194 & 0.187 & -0.099 & 0.092 & & 0.142 & 13 & D & 287.5 & D & 287.5 \\
\hline 367 & KAU009 & 22.87 & 120.26 & -0.230 & 0.163 & -0.339 & 0.217 & -0.378 & 0.063 & -0.373 & 0.109 & 7 & D & 270.5 & D & 270.5 \\
\hline 368 & KAU010 & 22.79 & 120.28 & -0.371 & 0.056 & 0.121 & 0.182 & -0.314 & 0.049 & & 0.142 & 10 & E & & & 289.2 \\
\hline 369 & KAU011 & 22.76 & 120.26 & 0.078 & 0.435 & 0.129 & 0.510 & 0.008 & 0.290 & 0.119 & 0.395 & 10 & E & 145.4 & $\mathrm{E}$ & 145.4 \\
\hline 370 & KAU012 & 22.88 & 120.37 & -0.053 & 0.223 & 0.053 & 0.207 & -0.090 & 0.135 & 0.057 & 0.137 & 22 & D & 304.7 & D & 304.7 \\
\hline 371 & & 22.80 & & & & & & & & & & 1 & 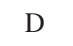 & & 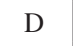 & \\
\hline 372 & KAU014 & 22.70 & 120.34 & -0.102 & 0.301 & 0.045 & 0.196 & -0.175 & 0.166 & 0.013 & 0.078 & 7 & E & 243.1 & D & 243.1 \\
\hline 373 & KAU015 & 22.66 & 120.33 & -0.121 & 0.099 & 0.206 & 0.164 & -0.127 & 0.073 & 0.211 & 0.100 & 10 & D & 227.1 & $\mathrm{D}$ & 227.1 \\
\hline 374 & & & & & & & & & & & & 1 & D & & * & \\
\hline 375 & KAU017 & 22.51 & 120.39 & -0.034 & 0.061 & 0.279 & 0.100 & -0.227 & 0.094 & 0.200 & 0.073 & 7 & E & 252.6 & D & 252.6 \\
\hline 376 & KAU018 & 22.89 & 120.47 & -0.605 & 0.251 & -0.332 & 0.213 & -0.666 & 0.141 & -0.357 & 0.125 & 13 & D & $* * * * *$ & $*$ & 743.0 \\
\hline 377 & KAU019 & 22.89 & 120.45 & -0.649 & 0.048 & -0.509 & & -0.651 & 0.023 & -0.380 & & 4 & $\mathrm{C}$ & $* * * * *$ & $*$ & 774.1 \\
\hline 378 & KAU020 & 22.90 & 120.52 & 0.126 & 0.314 & 0.373 & 0.273 & 0.139 & 0.216 & 0.413 & 0.183 & 20 & D & 344.2 & D & 344.2 \\
\hline 379 & KAU021 & 22.75 & 120.44 & -1.378 & 0.000 & -1.023 & 0.000 & -1.185 & 0.000 & -0.829 & 0.000 & 1 & $\mathrm{C}$ & & $*$ & 1740.0 \\
\hline 380 & KAU022 & 22.67 & 120.49 & -0.273 & 0.305 & 0.051 & 0.361 & -0.148 & 0.239 & 0.138 & 0.187 & 12 & $\mathrm{E}$ & $* * * * *$ & $*$ & 304.8 \\
\hline 381 & KAU023 & 22.68 & 120.49 & -0.578 & 0.001 & -0.247 & 0.098 & -0.595 & 0.010 & -0.374 & 0.049 & 2 & D & 218.2 & D & 218.2 \\
\hline 382 & & 22.66 & & & & & & -0.444 & & -0.433 & 0.140 & 3 & $\mathrm{E}$ & & D & 220.6 \\
\hline 383 & KAU025 & 22.68 & 120.48 & -0.437 & 0.131 & -0.109 & 0.208 & -0.482 & 0.030 & -0.154 & 0.082 & 7 & D & $* * * * *$ & $*$ & 515.9 \\
\hline 384 & KAU026 & 22.70 & 120.50 & -0.131 & 0.045 & 0.193 & 0.113 & -0.408 & 0.045 & 0.069 & 0.076 & 5 & $\mathrm{D}$ & 275.2 & D & 275.2 \\
\hline 385 & & 22.65 & & & & & & & & & & 3 & $\mathrm{E}$ & & $*$ & 620.0 \\
\hline 386 & KAU028 & 22.83 & 120.59 & -0.746 & & -0.645 & 0.267 & -0.694 & 0.040 & -0.661 & & 5 & D & 547.8 & $\mathrm{C}$ & 547.8 \\
\hline 387 & KAU029 & 22.76 & 120.57 & -0.204 & 0.031 & -0.056 & 0.005 & -0.536 & 0.031 & -0.121 & 0.017 & 2 & $\mathrm{D}$ & 351.9 & D & 351.9 \\
\hline 388 & & & & & & 0.1 & & & & & & 12 & E & & D & 5.2 \\
\hline 389 & KAU031 & 22.61 & 120.48 & -0.489 & 0.051 & -0.010 & 0.135 & -0.585 & 0.043 & -0.132 & 0.050 & 5 & $\mathrm{E}$ & 219.6 & D & 219.6 \\
\hline 390 & KAU032 & 22.55 & 120.45 & 0.025 & 0.131 & 0.360 & 0.164 & 0.049 & 0.127 & 0.382 & 0.105 & 12 & E & 190.7 & D & 190.7 \\
\hline 391 & KAU033 & 22.47 & 120.45 & -0.134 & 0.127 & 0.258 & 0.170 & -0.291 & 0.098 & 0.181 & 0.122 & 9 & E & 186.6 & D & 186.6 \\
\hline 392 & KAU034 & 22.53 & 120.62 & -0.768 & 0.143 & -0.610 & 0.051 & -0.758 & 0.002 & -0.750 & 0.032 & 3 & B & 999.2 & B & 999.2 \\
\hline 393 & KAU035 & 22.55 & 120.53 & 0.112 & 0.129 & 0.456 & 0.211 & -0.045 & 0.109 & 0.342 & 0.111 & 8 & E & 282.0 & D & 282.0 \\
\hline 394 & KAU036 & 22.47 & 120.54 & 0.058 & 0.272 & 0.323 & 0.256 & -0.063 & 0.121 & 0.248 & 0.147 & 12 & E & 234.6 & D & 234.6 \\
\hline 395 & KAU037 & 22.30 & 120.63 & -0.130 & 0.213 & -0.134 & 0.154 & -0.181 & 0.138 & -0.177 & 0.096 & 12 & D & 277.3 & D & 277.3 \\
\hline 396 & KAU038 & 22.19 & 120.69 & -0.549 & & -0.664 & & & 0.165 & & & 6 & B & 660.4 & $\mathrm{C}$ & 660.4 \\
\hline 397 & KAU039 & 22.10 & 120.74 & -0.120 & 0.067 & 0.013 & 0.010 & -0.307 & 0.000 & -0.195 & 0.013 & 2 & D & 459.5 & $\mathrm{C}$ & 459.5 \\
\hline 398 & KAU040 & 22.19 & 120.87 & 0.014 & 0.021 & 0.119 & 0.051 & -0.174 & 0.148 & -0.090 & 0.046 & 2 & $\mathrm{D}$ & $* * * * *$ & $*$ & 459.5 \\
\hline 399 & KAU042 & 22.02 & 120.83 & & & -0.028 & & -0.405 & 0.000 & -0.225 & & 1 & $\mathrm{D}$ & 815.6 & B & 815.6 \\
\hline 400 & KAU043 & 21.91 & 120.84 & 0.093 & 0.078 & 0.524 & 0.000 & 0.081 & 0.019 & 0.423 & 0.008 & 2 & D & $* * * * *$ & $*$ & 182.5 \\
\hline 401 & KAU044 & 22.44 & 120.50 & 0.165 & 0.189 & 0.537 & 0.166 & 0.077 & 0.144 & 0.474 & 0.146 & 11 & $\mathrm{E}$ & 215.9 & D & 215.9 \\
\hline
\end{tabular}


Table 1. (Continued)

\begin{tabular}{|c|c|c|c|c|c|c|c|c|c|c|c|c|c|c|c|c|}
\hline \multirow{3}{*}{ No. } & \multirow{3}{*}{$\begin{array}{l}\text { Stname } \\
\text { KAU045 }\end{array}$} & \multirow{3}{*}{$\begin{array}{c}\text { Lat } \\
\left({ }^{\circ} \mathbf{N}\right)\end{array}$} & \multirow{3}{*}{$\begin{array}{c}\text { Long } \\
\left({ }^{\circ} \mathbf{E}\right)\end{array}$} & \multicolumn{4}{|c|}{ TotalRes } & \multicolumn{4}{|c|}{ IntraRes } & \multirow{3}{*}{$\begin{array}{c}\mathbf{R} \\
10\end{array}$} & \multirow{3}{*}{$\frac{\text { Site } \dagger}{E}$} & \multirow{3}{*}{$\begin{array}{c}\begin{array}{c}\text { Vs30 } \\
\left(\mathbf{m ~ s}^{-1}\right)\end{array} \\
145.2\end{array}$} & \multirow{3}{*}{$\begin{array}{c}\text { Site } F \\
E\end{array}$} & \multirow{3}{*}{$\begin{array}{r}\begin{array}{r}\text { Vs30N } \\
\left(\mathbf{m ~ s}^{-1}\right)\end{array} \\
145.2\end{array}$} \\
\hline & & & & \multicolumn{2}{|c|}{ PGAh \pm sd } & \multicolumn{2}{|c|}{$P G V h \pm s d$} & TUA & $\pm \mathrm{sd}$ & PGV & \pm sd & & & & & \\
\hline & & & & -0.120 & 0.118 & 0.384 & 0.202 & -0.247 & 0.138 & 0.339 & 0.155 & & & & & \\
\hline 403 & KAU046 & 22.01 & 120.74 & 0.363 & 0.109 & 0.539 & .070 & 0.286 & 0.075 & 0.412 & 0.030 & 5 & D & 98.4 & D & 198.4 \\
\hline 404 & KAU047 & 23.08 & 120.58 & -0.346 & 0.333 & -0.356 & 0.165 & -0.395 & 0.138 & -0.397 & 0.086 & 19 & B & $* * * * *$ & $*$ & 799.5 \\
\hline 405 & KAU048 & 22.73 & 120.49 & -0.297 & 0.198 & 0.205 & 131 & -0.306 & 0.108 & 0.160 & 0.074 & 14 & D & 139.5 & E & 139.5 \\
\hline 406 & KAU049 & 22.75 & 120.63 & -0.006 & 0.128 & -0.336 & 0.233 & -0.131 & 0.413 & -0.420 & 0.326 & 6 & $\mathrm{C}$ & $* * * * *$ & $*$ & 833.2 \\
\hline 407 & KAU050 & 23.16 & 120.76 & -0.352 & 0.366 & -0.709 & 0.332 & -0.338 & 0.247 & -0.671 & 0.183 & 17 & B & $* * * * *$ & $*$ & 1308.7 \\
\hline 408 & KAU051 & 22.37 & 120.62 & -0.763 & 0.202 & -0.926 & 0.289 & -0.813 & 0.095 & -0.929 & 0.222 & 6 & B & 1019.5 & B & 1019.5 \\
\hline 409 & KAU053 & 22.82 & 120.42 & -0.470 & 0.252 & -0.160 & 0.107 & -0.609 & 0.121 & -0.237 & 0.055 & 7 & B & $* * * *$ & $*$ & 599.2 \\
\hline 410 & KAU054 & 23.28 & 120.71 & -0.484 & 0.253 & -0.733 & 0.214 & -0.488 & 0.206 & -0.724 & 0.131 & 26 & $\mathrm{D}$ & 502.8 & $\mathrm{C}$ & 502.8 \\
\hline 411 & KAU055 & 22.57 & 120.34 & -0.244 & 0.107 & 0.186 & 0.155 & -0.252 & 0.091 & 0.139 & 0.120 & 10 & E & $* * * * *$ & $*$ & 304.0 \\
\hline 412 & KAU056 & 22.55 & 120.34 & -0.269 & 0.093 & 0.078 & 0.150 & -0.424 & 0.060 & -0.002 & 0.123 & 9 & E & $* * * * *$ & $*$ & 392.2 \\
\hline 413 & KAU057 & 22.63 & 120.26 & -0.352 & 0.087 & -0.362 & 0.177 & -0.426 & 0.039 & -0.417 & 0.104 & 9 & $\mathrm{C}$ & $* * * * *$ & $*$ & 828.1 \\
\hline 414 & KAU058 & 22.64 & 120.32 & -0.163 & 0.129 & 0.151 & 0.130 & -0.151 & 0.076 & 0.115 & 0.103 & 11 & E & $* * * * *$ & $*$ & 317.6 \\
\hline 415 & KAU059 & 22.73 & 120.31 & -0.666 & 0.075 & -0.416 & 0.166 & -0.577 & 0.014 & -0.329 & 0.073 & 3 & $\mathrm{E}$ & 229.6 & D & 229.6 \\
\hline 416 & KAU060 & 22.67 & 120.31 & -0.020 & 0.000 & -0.036 & 0.000 & -0.352 & 0.000 & -0.039 & 0.000 & 1 & E & 246.0 & D & 246.0 \\
\hline 417 & KAU061 & 22.64 & 120.29 & 0.149 & 0.106 & 0.088 & 0.018 & 0.054 & 0.008 & 0.228 & 0.000 & 2 & $\mathrm{E}$ & 14.4 & D & 14.4 \\
\hline 418 & KAU062 & 22.62 & 120.28 & 0.048 & 0.136 & 0.063 & 0.227 & 0.083 & 0.079 & 0.105 & 0.143 & 10 & $\mathrm{E}$ & 195.6 & D & 195.6 \\
\hline 419 & KAU063 & 22.91 & 120.17 & -0.167 & 0.103 & 0.387 & 0.240 & -0.106 & 0.105 & 0.417 & 0.164 & 12 & E & 211.6 & D & 211.6 \\
\hline 420 & & 22.79 & & & & & & & & & & 13 & $\mathrm{E}$ & & $\Gamma_{1}$ & \\
\hline 421 & KAU065 & 22.75 & 120.30 & -0.291 & 0.003 & -0.313 & 0.000 & -0.044 & 0.003 & 0.021 & 0.002 & 2 & $\mathrm{E}$ & 244.9 & $\mathrm{D}$ & 244.9 \\
\hline 422 & KAU066 & 22.73 & 120.34 & 0.092 & 0.139 & 0.189 & 0.161 & 0.045 & 0.102 & 0.155 & 0.104 & 15 & E & 228.6 & D & 228.6 \\
\hline 423 & KAU067 & 22.66 & 120.42 & -0.409 & 0.000 & -0.101 & 0.000 & -0.741 & 0.000 & -0.1 & 0.000 & 1 & D & 463.6 & $\mathrm{C}$ & 463.6 \\
\hline 424 & KAU068 & 22.98 & 120.54 & 0.101 & 0.001 & -0.033 & 0.004 & -0.228 & 0.001 & -0.051 & 0.003 & 2 & D & 813.2 & B & 813.2 \\
\hline 425 & KAU069 & 22.89 & 120.66 & -0.062 & 0.376 & -0.643 & 0.299 & -0.081 & 0.109 & -0.625 & 0.123 & 14 & B & 12.7 & $\mathrm{C}$ & 512.7 \\
\hline 426 & KAU070 & 22.78 & 120.49 & -0.243 & 0.166 & 0.019 & 0.190 & -0.383 & 0.064 & -0.028 & 0.076 & 5 & D & 289.4 & D & 289.4 \\
\hline 427 & KAU071 & 22.66 & 120.51 & -0.154 & 0.020 & 0.210 & 0.033 & -0.477 & 0.027 & 0.025 & 0.035 & 3 & E & 238.7 & $\mathrm{D}$ & 238.7 \\
\hline 428 & KAU072 & 22.67 & 120.59 & -0.194 & 0.087 & -0.244 & 0.200 & -0.278 & 0.299 & -0.345 & 0.324 & 5 & $\mathrm{D}$ & 487.1 & $\mathrm{C}$ & 487.1 \\
\hline 429 & KAU073 & 22.53 & 120.53 & -0.384 & 0.477 & 0.053 & 0.406 & -0.393 & 0.314 & 0.057 & 0.319 & 15 & E & $* * * * *$ & $*$ & 352.4 \\
\hline 430 & KAU074 & 22.57 & 120.57 & -0.101 & 0.170 & 0.196 & 0.194 & -0.116 & 0.110 & 0.194 & 0.093 & 17 & E & 224.9 & D & 224.9 \\
\hline 431 & & 22.49 & & & & & & & & & & 14 & t & & $\mathrm{D}$ & \\
\hline 432 & KAU076 & 22.43 & 120.56 & 0. & 0.002 & 0.278 & 0.009 & -0.116 & 0.020 & 0.1 & 0.000 & 2 & D & 280.2 & $\mathrm{D}$ & 280.2 \\
\hline 433 & KAU077 & 22.75 & 120.72 & -0.349 & 0.269 & -0.672 & 0.386 & -0.334 & 0.242 & -0.667 & 0.284 & 16 & B & $* * * * *$ & $*$ & 1299.1 \\
\hline 434 & & 22.71 & & & & & & & & & & 17 & B & & $\mathrm{C}$ & 553.1 \\
\hline 435 & KAU079 & 22.59 & 120.62 & -0.297 & 0.083 & -0.801 & 0.165 & -0.422 & 0.144 & -0.8 & 0.180 & 6 & B & 593.8 & $\mathrm{C}$ & 593.8 \\
\hline 436 & KAU081 & 22.01 & 120.74 & 0.138 & 0.113 & 0.282 & 0.236 & 0.176 & 0.146 & 0.226 & 0.157 & 7 & D & $* * * * *$ & $*$ & 260.1 \\
\hline 437 & KAU082 & 21.94 & 120.73 & & & & 0.019 & 0.132 & 0.000 & & & 2 & $\mathrm{C}$ & & * & 174.8 \\
\hline 438 & KAU083 & 22.57 & 120.45 & -0.208 & 0.164 & 0.357 & 0.137 & -0.140 & 0.112 & 0.341 & 0.062 & 9 & E & $* * * * *$ & $*$ & 211.3 \\
\hline 439 & KAU084 & 22.35 & 120.36 & -0.121 & 0.000 & 0.527 & 0.000 & -0.548 & 0.000 & 0.330 & 0.000 & 1 & B & $* * * * *$ & $*$ & 215.6 \\
\hline 440 & KAU085 & 22.89 & 120.32 & & 0.211 & 0.450 & 0.241 & 0.046 & 0.134 & 0.465 & 0.129 & 15 & D & 255.8 & D & 255.8 \\
\hline 441 & KAU086 & 22.79 & 120.30 & 0.082 & 0.058 & 0.330 & 0.212 & 0.028 & 0.068 & 0.334 & 0.181 & 8 & E & $* * * * *$ & $*$ & 214.1 \\
\hline 442 & KAU087 & 22.61 & & & & & & & & & & 11 & $\mathrm{E}$ & & $\mathrm{D}$ & 47.4 \\
\hline 443 & KAU088 & 22.65 & 120.31 & & & & & -0.091 & 0.043 & & & 12 & E & 228.7 & $\mathrm{D}$ & 228.7 \\
\hline 444 & KAU089 & 22.48 & 120.39 & -0.209 & 0.130 & 0.405 & 0.261 & -0.198 & 0.119 & 0.396 & 0.198 & 12 & E & 191.5 & D & 191.5 \\
\hline 445 & ILA001 & 24.88 & & & & & & & & & & 9 & D & 939.1 & B & 939.1 \\
\hline 446 & ILA002 & 24.84 & 121.80 & 0.138 & 0.189 & 0.142 & 0.140 & 0.124 & 0.107 & 0.170 & 0.120 & 16 & D & 220.7 & D & 220.7 \\
\hline 447 & ILA003 & 24.80 & 121.78 & 0.232 & 0.159 & 0.267 & 0.090 & 0.253 & 0.083 & 0.270 & 0.063 & 14 & E & 265.6 & D & 265.6 \\
\hline 448 & ILA004 & 24.75 & 121.78 & 0.187 & 0.110 & 0.563 & 0.094 & 0.223 & 0.072 & 0.608 & 0.080 & 13 & $\mathrm{E}$ & 121.5 & E & 121.5 \\
\hline 449 & ILA005 & 24.70 & 121.80 & & 0.212 & 0.311 & 0.194 & 0.275 & 0.106 & 0.296 & 0.153 & 20 & $\mathrm{E}$ & 237.2 & D & 237.2 \\
\hline 450 & ILA006 & 24.64 & 121.82 & 0.303 & 0.171 & 0.327 & 0.229 & 0.274 & 0.116 & 0.304 & 0.220 & 21 & E & 276.4 & $\mathrm{D}$ & 276.4 \\
\hline 451 & ILA007 & 24.59 & 121.85 & 0.320 & 0.300 & 0.029 & 0.267 & 0.309 & 0.228 & 0.013 & 0.279 & 23 & D & $* * * * *$ & $*$ & 381.7 \\
\hline 452 & ILA008 & 24.71 & 121.76 & 0.176 & 0.130 & 0.227 & 0.170 & 0.147 & 0.092 & 0.205 & 0.152 & 21 & $\mathrm{E}$ & 290.7 & $\mathrm{D}$ & 290.7 \\
\hline 453 & ILA010 & 24.63 & 121.78 & & 0.294 & -0.348 & 0.328 & -0.047 & 0.158 & -0.382 & 0.284 & 13 & D & & $*$ & 777.9 \\
\hline 454 & ILA011 & 24.83 & 121.75 & -0.559 & 0.000 & -0.952 & 0.000 & -0.421 & 0.000 & -0.800 & 0.000 & 1 & B & $* * * * *$ & $*$ & 1651.4 \\
\hline 455 & ILA012 & 24.78 & 121.73 & 0.165 & 0.112 & 0.298 & 0.126 & 0.141 & 0.070 & 0.273 & 0.112 & 16 & $\mathrm{D}$ & 255.5 & D & 255.5 \\
\hline
\end{tabular}


Table 1. (Continued)

\begin{tabular}{|c|c|c|c|c|c|c|c|c|c|c|c|c|c|c|c|c|}
\hline \multirow{3}{*}{$\begin{array}{l}\text { No. } \\
456\end{array}$} & \multirow{3}{*}{$\begin{array}{l}\text { Stname } \\
\text { ILA013 }\end{array}$} & \multirow{3}{*}{$\begin{array}{c}\begin{array}{c}\text { Lat } \\
\left({ }^{\circ} \mathbf{N}\right)\end{array} \\
24.73 \\
\end{array}$} & \multirow{3}{*}{$\begin{array}{c}\begin{array}{c}\text { Long } \\
\left({ }^{\circ} \mathbf{E}\right)\end{array} \\
121.73\end{array}$} & \multicolumn{4}{|c|}{ TotalRes } & \multicolumn{4}{|c|}{ IntraRes } & \multirow{3}{*}{$\begin{array}{l}\mathbf{R} \\
16\end{array}$} & \multirow{3}{*}{$\frac{\text { Site } \dagger}{D}$} & \multirow{3}{*}{$\begin{array}{c}\begin{array}{c}\text { Vs30 } \\
\left(\mathbf{m ~ s}^{-1}\right)\end{array} \\
194.5\end{array}$} & \multirow{3}{*}{$\frac{\text { Site }+}{D}$} & \multirow{3}{*}{$\begin{array}{r}\begin{array}{r}\text { Vs30N } \\
\left(\mathbf{m ~ s}^{-1}\right)\end{array} \\
194.5\end{array}$} \\
\hline & & & & \multicolumn{2}{|c|}{ PGAh \pm sd } & \multicolumn{2}{|c|}{ PGVh \pm sd } & PGA & $\pm s d$ & PGV & $\pm s d$ & & & & & \\
\hline & & & & 0.591 & 0.344 & 0.648 & 0.207 & 0.626 & 0.137 & 0.673 & 0.140 & & & & & \\
\hline 457 & ILA014 & 24.69 & 121.72 & 0.132 & 0.269 & 0.140 & & & & 0.114 & 0.236 & 14 & D & 307.5 & D & 307.5 \\
\hline 458 & ILA015 & 24.78 & 121.69 & -0.322 & 0.107 & -0.512 & .114 & -0.385 & 0.062 & -0.515 & 0.079 & 16 & B & 776.8 & B & 776.8 \\
\hline 459 & ILA016 & 24.75 & 121.68 & 0.004 & 0.239 & 0.116 & 0.200 & 0.017 & 0.120 & 0.130 & 0.153 & 20 & D & 269.7 & D & 269.7 \\
\hline 460 & ILA017 & 24.72 & 121.68 & -0.560 & 0.300 & -0.169 & 0.469 & -0.482 & 0.244 & -0.035 & & 7 & D & 627.4 & $\mathrm{C}$ & 627.4 \\
\hline 461 & ILA018 & 24.68 & 121.68 & -0.520 & 0.066 & -0.477 & 0.215 & -0.524 & 0.086 & -0.433 & 0.103 & 4 & D & 497.5 & $\mathrm{C}$ & 497.5 \\
\hline 462 & ILA019 & 24.64 & 121.69 & -0.049 & 0.052 & -0.158 & 0.126 & -0.179 & 0.077 & -0.324 & 0.114 & 5 & B & $* * * * *$ & $*$ & 700.0 \\
\hline 463 & ILA020 & 24.76 & 121.63 & 0.264 & 0.000 & 0.193 & 0.000 & 0.036 & 0.000 & -0.196 & 0.000 & 1 & B & 453.6 & $\mathrm{C}$ & 453.6 \\
\hline 464 & ILA021 & 24.71 & 121.64 & -0.335 & 0.274 & -0.360 & 0.214 & -0.330 & 0.140 & -0.338 & 0.157 & 15 & D & $* * * * *$ & $*$ & 718.0 \\
\hline 465 & ILA022 & 24.67 & 121.64 & -0.498 & 0.242 & -0.465 & 0.488 & -0.534 & 0.200 & -0.373 & 0.330 & 6 & D & $* * * * *$ & $*$ & 765.5 \\
\hline 466 & ILA023 & 24.68 & 121.60 & -0.164 & 0.230 & -0.453 & 0.327 & -0.196 & 0.185 & -0.429 & 0.172 & 9 & D & $* * * * *$ & * & 845.9 \\
\hline 467 & ILA024 & 24.64 & 121.59 & -0.619 & 0.237 & -0.859 & 0.346 & -0.671 & 0.217 & -0.787 & 0.240 & 14 & B & $* * * * *$ & * & 1612.6 \\
\hline 468 & ILA025 & 24.64 & 121.57 & 0.494 & 0.283 & -0.158 & & & & & & 9 & B & $* * * *$ & $*$ & 507.0 \\
\hline 469 & ILA026 & 24.67 & 121.76 & 0.137 & 0.297 & 0.401 & 0.127 & 0.177 & 0.065 & 0.451 & 0.063 & 4 & D & 237.5 & D & 237.5 \\
\hline 470 & ILA027 & 24.69 & 121.76 & 0.281 & 0.080 & 0.297 & 0.144 & 0.302 & 0.044 & 0.479 & 0.156 & 3 & D & 12.7 & D & 212.7 \\
\hline 471 & ILA028 & 24.76 & 121.75 & 0.635 & 0.170 & & 0.186 & & 0.079 & 0.835 & & 5 & D & 4 & D & 8.4 \\
\hline 472 & ILA029 & 24.77 & 121.75 & -0.312 & 0.317 & -0.005 & 0.267 & -0.289 & 0.230 & 0.118 & 0.288 & 4 & D & 187.0 & D & 187.0 \\
\hline 473 & ILA030 & 24.73 & 121.76 & 0.374 & 0.253 & 0.499 & 0.194 & 0.410 & 0.130 & 0.534 & 0.145 & 14 & E & .5 & D & 198.5 \\
\hline 474 & ILA031 & 24.60 & 121.83 & 0.387 & 0.211 & -0.122 & 0.248 & 0.368 & 0.159 & -0.083 & 0.231 & 16 & B & 657.4 & $\mathrm{C}$ & 657.4 \\
\hline 475 & ILA032 & 24.62 & 121.83 & 0.092 & 0.160 & -0.027 & 0.118 & 0.114 & 0.133 & 0.155 & 0.067 & 3 & D & 296.5 & D & 296.5 \\
\hline 476 & ILA033 & 24.86 & 121.82 & 0.265 & 0.118 & 0.083 & 0.134 & 0.166 & 0.053 & 0.114 & 0.092 & 5 & D & & D & 253.6 \\
\hline 477 & ILA034 & 24.80 & 121.81 & -0.303 & 0.253 & 0.401 & 0.239 & -0.199 & 0.061 & 0.470 & 0.028 & 3 & $\mathrm{E}$ & 217.7 & $\mathrm{D}$ & 217.7 \\
\hline 478 & ILA035 & 24.82 & 121.76 & -0.274 & 0.353 & -0.109 & 0.161 & -0.252 & 0.338 & 0.073 & 0.151 & 3 & D & 293.1 & D & 293.1 \\
\hline 479 & & & & & & & & & & & & 18 & 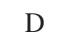 & & 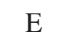 & \\
\hline 480 & ILA037 & 24.75 & 121.71 & 0.272 & 0.184 & 0.357 & 0.190 & 0.319 & 0.068 & 0.402 & 0.128 & 19 & D & 212.9 & D & 212.9 \\
\hline 481 & ILA038 & 24.72 & 121.73 & 0.061 & 0.081 & 0.157 & 0.171 & 0.082 & 0.082 & 0.339 & 0.141 & 3 & D & 243.9 & D & 243.9 \\
\hline 482 & ILAO & & & & & & & & & & & 3 & D & & 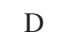 & \\
\hline 483 & ILA040 & 24.77 & 121.79 & 0.182 & 0.340 & 0.360 & 0.190 & 0.223 & 0.113 & 0.411 & 0.182 & 4 & E & 188.4 & D & 188.4 \\
\hline 484 & ILA041 & 24.72 & 121.79 & 0.370 & 0.132 & 0.516 & 0.138 & 0.364 & 0.043 & 0.478 & 0.090 & 14 & E & 194.2 & D & 194.2 \\
\hline 485 & ILA042 & 24.69 & 121.79 & 0.176 & 0.232 & 0.271 & & & 0.102 & 0.298 & 0.187 & 12 & E & 213.2 & D & 213.2 \\
\hline 486 & ILA044 & 24.66 & 121.76 & 0.192 & 0.258 & 0.447 & 0.164 & 0.227 & 0.159 & 0.452 & 0.150 & 11 & $\mathrm{D}$ & 159.0 & E & 159.0 \\
\hline 487 & ILA046 & 24.67 & 121.73 & -0.004 & 0.281 & -0.060 & 0.264 & -0.001 & 0.161 & -0.025 & 0.184 & 18 & D & 397.6 & $\mathrm{C}$ & 397.6 \\
\hline 488 & ILA047 & 24.64 & 121.79 & 0.056 & 0.003 & 0.281 & 0.044 & 0.091 & 0.134 & 0.398 & 0.154 & 4 & D & $* * * * *$ & $*$ & 190.7 \\
\hline 489 & ILA048 & 24.77 & 121.76 & 0.474 & 0.292 & 0.616 & 0.157 & 0.553 & 0.109 & 0.636 & 0.120 & 15 & $\mathrm{E}$ & 192.1 & D & 192.1 \\
\hline 490 & & 24.77 & & & & & & & & & & 16 & D & & D & 187.1 \\
\hline 491 & ILA050 & 24.43 & 121.74 & & 0.565 & -0.521 & 0.437 & 0.345 & 0.495 & -0.456 & 0.461 & 17 & B & 626.6 & $\mathrm{C}$ & 626.6 \\
\hline 492 & ILA051 & 24.72 & 121.67 & -0.222 & 0.265 & -0.358 & 0.244 & -0.234 & 0.175 & -0.307 & 0.227 & 17 & B & 535.2 & $\mathrm{C}$ & 535.2 \\
\hline 493 & & 24.61 & & & & -0.668 & & & & & & 15 & B & & 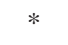 & 1297.4 \\
\hline 494 & ILA053 & 24.33 & 121.73 & & 0.106 & -0.131 & & 0.083 & 0.096 & -0.200 & 0.150 & 11 & D & 534.7 & $\mathrm{C}$ & 534.7 \\
\hline 495 & ILA054 & 24.97 & 121.92 & -0.501 & 0.116 & -0.721 & 0.158 & -0.553 & 0.109 & -0.670 & 0.125 & 10 & B & 783.1 & B & 783.1 \\
\hline 496 & ILA055 & 24.74 & & & & & & & & & & 21 & E & 0 & D & 266.0 \\
\hline 497 & ILA056 & 24.76 & 121.81 & 0.110 & 0.176 & 0.437 & 0.176 & 0.108 & 0.096 & 0.445 & 0.141 & 15 & $\mathrm{E}$ & 221.3 & D & 221.3 \\
\hline 498 & ILA057 & 24.81 & 121.74 & -0.343 & 0.283 & -0.897 & 0.156 & -0.347 & 0.082 & -0.773 & 0.081 & 3 & B & $* * * * *$ & $*$ & 1572.2 \\
\hline 499 & ILA058 & 24.68 & 121.75 & 0.314 & 0.162 & 0.406 & 0.107 & 0.292 & 0.155 & 0.353 & 0.083 & 11 & D & $* * * * *$ & $*$ & 206.8 \\
\hline 500 & ILA059 & 24.67 & 121.82 & 0.211 & 0.191 & 0.243 & 0.150 & 0.193 & 0.145 & 0.213 & 0.164 & 18 & E & 232.2 & D & 232.2 \\
\hline 501 & ILA060 & 24.58 & 121.84 & 0.052 & 0.194 & -0.436 & 0.211 & 0.003 & 0.196 & -0.438 & 0.252 & 10 & D & $* * * * *$ & $*$ & 860.7 \\
\hline 502 & ILA061 & 24.52 & 121.83 & -0.342 & 0.166 & -0.570 & 0.124 & -0.372 & 0.115 & -0.540 & 0.137 & 15 & D & 502.1 & $\mathrm{C}$ & 502.1 \\
\hline 503 & ILA062 & 24.47 & 121.79 & 0.183 & 0.295 & -0.167 & 0.213 & 0.172 & 0.137 & -0.184 & 0.140 & 20 & D & $* * * * *$ & $*$ & 543.8 \\
\hline 504 & ILA063 & 24.61 & 121.52 & -0.341 & 0.183 & -0.783 & & -0.437 & 0.151 & & 0.090 & 14 & B & 1002.7 & & 1002.7 \\
\hline 505 & ILA064 & 24.48 & 121.78 & -0.102 & 0.253 & -0.533 & 0.190 & -0.074 & 0.164 & -0.531 & 0.165 & 14 & D & $* * * * *$ & $*$ & 1017.8 \\
\hline 506 & ILA065 & 24.47 & 121.77 & 0.703 & 0.470 & 0.000 & 0.295 & 0.703 & 0.416 & 0.055 & 0.198 & 11 & $\mathrm{D}$ & $* * * * *$ & $*$ & 354.2 \\
\hline 507 & ILA066 & 24.45 & 121.77 & 0.311 & & -0.089 & & & 0.119 & -0.083 & & 21 & D & 477.6 & $\mathrm{C}$ & 477.6 \\
\hline 508 & ILA067 & 24.44 & 121.37 & -0.121 & 0.334 & -0.497 & 0.325 & 0.126 & 0.134 & -0.363 & 0.274 & 9 & B & $* * * * *$ & $*$ & 751.1 \\
\hline 509 & HWA001 & 23.79 & 121.56 & -0.149 & 0.320 & -0.262 & 0.343 & -0.184 & 0.273 & -0.180 & 0.270 & 9 & D & 565.2 & $\mathrm{C}$ & 565.2 \\
\hline
\end{tabular}


Table 1. (Continued)

\begin{tabular}{|c|c|c|c|c|c|c|c|c|c|c|c|c|c|c|c|c|}
\hline \multirow{3}{*}{ No. } & \multirow{3}{*}{$\begin{array}{c}\text { Stname } \\
\text { HWA002 }\end{array}$} & \multirow{3}{*}{$\begin{array}{c}\begin{array}{c}\text { Lat } \\
\left({ }^{\circ} \mathbf{N}\right)\end{array} \\
23.60\end{array}$} & \multirow{3}{*}{$\begin{array}{c}\text { Long } \\
\left({ }^{\circ} \mathbf{E}\right)\end{array}$} & \multicolumn{4}{|c|}{ TotalRes } & \multicolumn{4}{|c|}{ IntraRes } & \multirow{3}{*}{$\begin{array}{l}\mathbf{R} \\
16\end{array}$} & \multirow{3}{*}{$\frac{\text { Site } \uparrow}{\mathrm{C}}$} & \multirow{3}{*}{$\begin{array}{c}\begin{array}{c}\text { Vs30 } \\
\left(\mathbf{m ~ s}^{-1}\right)\end{array} \\
789.4\end{array}$} & \multirow{3}{*}{$\frac{\text { Site }+}{\text { B }}$} & \multirow{3}{*}{$\begin{array}{r}\begin{array}{r}\text { Vs30N } \\
\left(\mathbf{m ~ s}^{-1}\right)\end{array} \\
789.4\end{array}$} \\
\hline & & & & \multicolumn{2}{|c|}{ PGAh \pm sd } & \multicolumn{2}{|c|}{ PGVh \pm sd } & (1) & $\pm \mathrm{sd}$ & PGV & $\pm \mathrm{sd}$ & & & & & \\
\hline & & & & -0.756 & 0.210 & -0.660 & 0.235 & -0.762 & 0.115 & -0.649 & 0.214 & & & & & \\
\hline 511 & HWA003 & 23.48 & 121.50 & -0.664 & 0.258 & -0.235 & 227 & -0.782 & 0.113 & -0.276 & 0.183 & 7 & D & 1538.0 & & 1538.0 \\
\hline 512 & WA004 & 23.17 & 121.24 & -0.365 & 0.232 & -0.491 & 0.217 & -0.484 & & & & 9 & $\mathrm{C}$ & 319.6 & D & 319.6 \\
\hline 513 & HWA005 & 23.66 & 121.41 & -0.033 & 0.352 & -0.103 & 209 & -0.059 & 0.199 & -0.095 & 0.139 & 25 & D & & $*$ & 463.4 \\
\hline 514 & HWA006 & 23.67 & 121.42 & -0.546 & 0.265 & -0.752 & .245 & -0.439 & 0.169 & -0.660 & 0.207 & 9 & $\mathrm{D}$ & 557.1 & $\mathrm{C}$ & 557.1 \\
\hline 515 & HWA007 & 23.99 & 121.62 & -0.462 & 0.399 & -0.161 & 0.294 & -0.492 & 0.241 & -0.136 & & 20 & D & & $*$ & 499.4 \\
\hline 516 & HWA008 & 23.99 & 121.60 & 0.004 & 0.435 & 0.264 & .308 & -0.035 & 0.241 & 0.281 & 0.177 & 15 & D & 297.0 & $\mathrm{D}$ & 297.0 \\
\hline 517 & HWA009 & 23.99 & 121.62 & -0.332 & 0.374 & -0.223 & 0.289 & -0.336 & 0.227 & -0.204 & 48 & 25 & $\mathrm{D}$ & & * & 564.0 \\
\hline 518 & HWA010 & 23.98 & 121.60 & -0.040 & 0.722 & 0.148 & 0.414 & -0.072 & 0.475 & 0.159 & & 12 & $\mathrm{D}$ & 249.7 & $\mathrm{D}$ & 249.7 \\
\hline 519 & IWA011 & 24.00 & 121.59 & 0.016 & 0.450 & 0.123 & 0.354 & 0.054 & 0.183 & 0.150 & 0.232 & 21 & D & 360.8 & $\mathrm{C}$ & 360.8 \\
\hline 520 & HWA012 & 23.99 & 121.62 & -0.492 & 0.469 & -0.220 & 0.354 & & 0.248 & & & 23 & $\mathrm{D}$ & 409.8 & $\mathrm{C}$ & 409.8 \\
\hline 521 & HWA013 & 23.98 & 121.59 & -0.042 & 0.437 & 0.151 & 0.302 & -0.020 & 0.208 & 0.183 & 0.195 & 26 & $\mathrm{D}$ & 336.8 & D & 336.8 \\
\hline 522 & HWA014 & 23.97 & 121.60 & -0.187 & 0.412 & 0.121 & .297 & -0.195 & 0.220 & & & 25 & $\mathrm{D}$ & & $*$ & 311.2 \\
\hline 523 & HWA015 & 23.97 & 121.56 & -0.390 & 0.561 & -0.359 & 0.439 & -0.367 & 0.289 & -0.325 & 00 & 25 & $\mathrm{D}$ & 445.6 & $\mathrm{C}$ & 445.6 \\
\hline 524 & HWA016 & 23.97 & 121.56 & -0.557 & 0.606 & -0.473 & 0.491 & -0.536 & 0.330 & -0.424 & & 24 & D & 579.6 & $\mathrm{C}$ & 579.6 \\
\hline 525 & HWA017 & 23.95 & 121.55 & -0.344 & 0.740 & -0.534 & 0.559 & -0.376 & 0.424 & -0. & & 20 & $\mathrm{D}$ & 84.7 & $\mathrm{C}$ & 584.7 \\
\hline 526 & HWA018 & 23.91 & 121.52 & -0.518 & 0.333 & -0.542 & 0.185 & -0.557 & 0.180 & -0.541 & & 17 & $\mathrm{D}$ & 462.6 & $\mathrm{C}$ & 462.6 \\
\hline 527 & HWA019 & 23.98 & 121.61 & -0.006 & 0.536 & & 0.437 & 0.012 & & & & 27 & $\mathrm{D}$ & 503.5 & $\mathrm{C}$ & 503.5 \\
\hline 528 & & & & & & & & & & & & 25 & 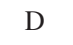 & & . & 9.6 \\
\hline 529 & HWA021 & 23.51 & 121.32 & -0.610 & 0.334 & -1.114 & 0.199 & -0.647 & 0.248 & -1.096 & 0.203 & 11 & B & $* * * * *$ & $*$ & 2813.5 \\
\hline 530 & HWA022 & 24.27 & 121.73 & 0.207 & 0.208 & -0.037 & 0.297 & 0.283 & 0.173 & 0.032 & 0.352 & 17 & B & & $*$ & 369.0 \\
\hline 531 & & 24.08 & & & & & & & & & & 18 & B & & $*$ & 171.0 \\
\hline 532 & HWA024 & 23.35 & 121.30 & -1.044 & 0.199 & -0.996 & 0.169 & -1.059 & 0.040 & -1.005 & 96 & 14 & B & $* * * * *$ & $*$ & 2390.2 \\
\hline 533 & HWA025 & 24.16 & 121.64 & -0.445 & 0.275 & -0.594 & 0.255 & -0.412 & 0.245 & -0. & 7 & 22 & D & 483.1 & $\mathrm{C}$ & 483.1 \\
\hline 534 & HWA & 24.13 & & & & & & & & & & 24 & B & 460.9 & $\mathrm{C}$ & 460.9 \\
\hline 535 & HWA027 & 24.06 & 121.59 & -0.197 & 0.541 & -0.392 & 0.478 & -0.178 & 0.293 & -0.347 & & 24 & D & 434.6 & $\mathrm{C}$ & 434.6 \\
\hline 536 & HWA028 & 24.02 & 121.60 & -0.103 & 0.448 & -0.034 & 0.389 & -0.082 & 0.231 & -0.001 & 0.311 & 25 & D & 404.9 & $\mathrm{C}$ & 404.9 \\
\hline 537 & HWA029 & 23.94 & 121.57 & -0.478 & 0.499 & -0.383 & 0.327 & -0.474 & 0.242 & -0.348 & & 22 & D & 597.1 & $\mathrm{C}$ & 597.1 \\
\hline 538 & HWA030 & 23.78 & 121.45 & -0.651 & 0.739 & -0.668 & 0.271 & -0.731 & 0.526 & -0.701 & 0.256 & 21 & $\mathrm{D}$ & 594.1 & $\mathrm{C}$ & 594.1 \\
\hline 539 & & & & & & & & & & & & 25 & 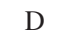 & & 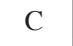 & \\
\hline 540 & HWA032 & 23.71 & 121.41 & -0.278 & 0.566 & -0.687 & 0.313 & -0.302 & 0.252 & -0.697 & & 20 & $\mathrm{D}$ & $* * * * *$ & $*$ & 1372.7 \\
\hline 541 & HWA033 & 23.69 & 121.47 & -0.315 & 0.308 & -0.217 & 0.233 & -0.285 & 0.148 & -0.179 & 0.182 & 26 & $\mathrm{C}$ & 392.8 & $\mathrm{C}$ & 392.8 \\
\hline 542 & & 23.59 & & & & & & & & & & 23 & D & & $\mathrm{C}$ & 81.8 \\
\hline 543 & HWA035 & 23.73 & 121.44 & -0.552 & 0.405 & -0.742 & 0.3 & -0.565 & 0.283 & -0.7 & & 23 & D & 686.1 & $\mathrm{C}$ & 686.1 \\
\hline 544 & HWA036 & 23.50 & 121.37 & -0.684 & 0.216 & -0.414 & 0.164 & -0.723 & 0.089 & -0.423 & 0.115 & 17 & $\mathrm{D}$ & 520.6 & $\mathrm{C}$ & 520.6 \\
\hline 545 & & & & & & & & & & & & 20 & D & & $\mathrm{C}$ & 66.5 \\
\hline 546 & HWA038 & 23.46 & 121.34 & -0.957 & 0.258 & -0.791 & 0.217 & -1.007 & 0.135 & -0.837 & 0.1 & 18 & $\mathrm{C}$ & 661.5 & $\mathrm{C}$ & 661.5 \\
\hline 547 & HWA039 & 23.38 & 121.35 & -0.443 & 0.219 & -0.232 & 0.138 & -0.468 & 0.084 & -0.249 & 0.103 & 18 & D & $* * * * *$ & $*$ & 612.4 \\
\hline 548 & HWA040 & 23.34 & 121.31 & -0.373 & 0.138 & -0.116 & 0.190 & -0.298 & 0.208 & -0.082 & & 5 & D & 438.6 & $\mathrm{C}$ & 438.6 \\
\hline 549 & HWA041 & 23.27 & 121.29 & -0.081 & 0.251 & -0.076 & 0.261 & -0.152 & 0.242 & -0.106 & 0.238 & 15 & D & 481.9 & $\mathrm{C}$ & 481.9 \\
\hline 550 & HWA042 & 23.22 & 121.26 & -0.398 & & & & -0.370 & 0.438 & -0.306 & 0.348 & 6 & $\mathrm{D}$ & & * & 677.5 \\
\hline 551 & HWA043 & 23.71 & 121.54 & & 0.386 & -0.678 & & -0.646 & 0.234 & -0.665 & & 23 & D & 544.5 & $\mathrm{C}$ & 544.5 \\
\hline 552 & HWA044 & 23.65 & 121.53 & -0.793 & 0.362 & -0.706 & 0.222 & -0.777 & 0.208 & -0.674 & 0.193 & 18 & $\mathrm{D}$ & 533.1 & $\mathrm{C}$ & 533.1 \\
\hline 553 & & 24.31 & & & & & & & & & & 17 & D & & $\mathrm{C}$ & 472.8 \\
\hline 554 & HWA046 & 24.15 & 121.62 & -0.520 & 0.271 & -0.705 & 0.108 & -0.462 & 0.100 & -0.668 & & 8 & B & 643.7 & $\mathrm{C}$ & 643.7 \\
\hline 555 & HWA047 & 24.13 & 121.65 & 0.430 & 0.477 & 0.278 & 0.328 & 0.223 & 0.538 & 0.224 & 0.275 & 4 & D & 543.7 & $\mathrm{C}$ & 543.7 \\
\hline 556 & & 24.01 & & & & & & & & & & 13 & $\mathrm{D}$ & & $\mathrm{D}$ & 349.8 \\
\hline 557 & HWA049 & 24.00 & 121.56 & -0.217 & 0.302 & -0.017 & 0.268 & -0.196 & 0.135 & -0.015 & 0.193 & 19 & D & 509.3 & $\mathrm{C}$ & 509.3 \\
\hline 558 & HWA050 & 23.99 & 121.58 & -0.401 & 0.413 & -0.517 & 0.352 & -0.405 & 0.164 & -0.510 & 0.232 & 16 & D & $* * * * *$ & $*$ & 978.5 \\
\hline 559 & HWA051 & 23.87 & 121.55 & -0.169 & 0.273 & -0.270 & 0.157 & -0.199 & 0.134 & -0.293 & 0.106 & 18 & D & 449.7 & $\mathrm{C}$ & 449.7 \\
\hline 560 & HWA052 & 23.82 & 121.47 & -0.730 & 0.361 & -0.926 & 0.288 & -0.735 & 0.168 & -0.826 & 0.111 & 4 & D & 576.5 & $\mathrm{C}$ & 576.5 \\
\hline 561 & HWA055 & 23.32 & 121.33 & -0.187 & 0.252 & 0.027 & 0.301 & -0.225 & 0.049 & -0.011 & 0.179 & 11 & $\mathrm{D}$ & $* * * * *$ & $*$ & 398.7 \\
\hline 562 & HWA056 & 24.18 & 121.51 & -0.077 & 0.363 & -0.557 & 0.271 & -0.064 & 0.230 & -0.516 & 0.287 & 22 & B & 516.4 & $\mathrm{C}$ & 990.0 \\
\hline 563 & HWA057 & 24.16 & 121.61 & -0.146 & 0.386 & -0.874 & 0.155 & -0.150 & 0.271 & -0.872 & 0.185 & 18 & B & $* * * * *$ & $*$ & 1878.9 \\
\hline
\end{tabular}


Table 1. (Continued)

\begin{tabular}{|c|c|c|c|c|c|c|c|c|c|c|c|c|c|c|c|c|}
\hline \multirow{3}{*}{ No. } & \multirow{3}{*}{$\begin{array}{l}\text { Stname } \\
\text { HWA058 }\end{array}$} & \multirow{3}{*}{$\begin{array}{c}\begin{array}{c}\text { Lat } \\
\left({ }^{\circ} \mathbf{N}\right)\end{array} \\
23.97\end{array}$} & \multirow{3}{*}{$\begin{array}{c}\text { Long } \\
\left({ }^{\circ} \mathbf{E}\right)\end{array}$} & \multicolumn{4}{|c|}{ TotalRes } & \multicolumn{4}{|c|}{ IntraRes } & \multirow{3}{*}{$\begin{array}{c}\mathbf{R} \\
18\end{array}$} & \multirow{3}{*}{$\frac{\text { Site } \dagger}{\mathrm{B}}$} & \multirow{3}{*}{$\begin{array}{c}\begin{array}{c}\text { Vs30 } \\
\left(\mathbf{m ~ s}^{-1}\right)\end{array} \\
525.7\end{array}$} & \multirow{3}{*}{$\frac{\text { Site } F}{\mathrm{C}}$} & \multirow{3}{*}{$\begin{array}{r}\begin{array}{r}\text { Vs30N } \\
\left(\mathbf{m ~ s}^{-1}\right)\end{array} \\
525.7\end{array}$} \\
\hline & & & & \multicolumn{2}{|c|}{ PGAh \pm sd } & \multicolumn{2}{|c|}{$P G V h \pm s d$} & 40 & $\pm s d$ & PGV & $\pm \mathrm{sd}$ & & & & & \\
\hline & & & & -0.481 & 0.278 & -0.801 & 0.210 & -0.441 & 0.192 & -0.798 & 0.161 & & & & & \\
\hline 565 & HWA059 & 23.87 & 121.51 & -0.282 & 0.281 & -0.496 & 225 & -0.262 & 0.100 & -0.494 & 0.171 & 19 & D & 209.7 & $\mathrm{D}$ & 209.7 \\
\hline 566 & WA060 & 23.87 & 121.60 & -1.141 & 0.163 & -1.166 & 0.125 & -1.234 & 0.081 & -1.145 & 0.064 & 12 & $\mathrm{C}$ & & $*$ & 3072.1 \\
\hline 567 & HWA061 & 24.02 & 121.60 & -0.108 & 0.094 & -0.098 & 126 & -0.125 & 0.087 & -0.099 & 0.103 & 7 & D & $* * * * *$ & $*$ & 466.7 \\
\hline 568 & TTN001 & 23.32 & 121.44 & -0.443 & 0.305 & -0.272 & 0.131 & -0.481 & 0.190 & -0.286 & 0.066 & 16 & $\mathrm{D}$ & 484.5 & $\mathrm{C}$ & 484.5 \\
\hline 569 & TTN002 & 22.97 & 121.30 & -0.276 & 0.534 & -0.448 & 0.266 & -0.339 & 0.550 & -0.426 & 0.309 & 10 & D & 663.9 & $\mathrm{C}$ & 663.9 \\
\hline 570 & TTN003 & 22.61 & 121.00 & -0.388 & 0.158 & -0.594 & 0.221 & -0.468 & 0.061 & -0.661 & 0.134 & 10 & D & 508.5 & $\mathrm{C}$ & 508.5 \\
\hline 571 & TTN004 & 22.91 & 121.13 & -0.066 & 0.238 & 0.033 & 76 & & 13 & 0.012 & 32 & 17 & D & & 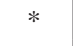 & 382.2 \\
\hline 572 & TTN005 & 22.76 & 121.14 & -0.603 & 0.122 & -0.196 & 0.126 & -0.617 & 0.108 & -0.131 & 0.084 & 10 & D & $* * * * *$ & $*$ & 494.5 \\
\hline 573 & TTN006 & 22.77 & 121.14 & -0.429 & 0.252 & 0.033 & 0.154 & -0.405 & 0.267 & 0.050 & 0.129 & 11 & D & $* * * * *$ & $*$ & 357.0 \\
\hline 574 & TTN007 & 22.76 & 121.14 & -0.417 & 0.227 & -0.004 & & & 0.197 & & & 9 & D & & $*$ & 69.9 \\
\hline 575 & TTN008 & 22.76 & 121.15 & -0.499 & 0.091 & 0.073 & 0.122 & -0.520 & 0.144 & 0.069 & 0.084 & 11 & D & $* * * * *$ & * & 345.0 \\
\hline 576 & TTN009 & 22.75 & 121.13 & -0.529 & 0.125 & -0.118 & 0.224 & -0.584 & 0.123 & -0.140 & & 14 & D & $* * * * *$ & $*$ & 502.9 \\
\hline 577 & TTN010 & 22.74 & 121.11 & -0.498 & 0.142 & -0.165 & 0.118 & -0.576 & 0.086 & -0.201 & 0.098 & 11 & $\mathrm{D}$ & $* * * * *$ & $*$ & 560.8 \\
\hline 578 & TTN011 & 22.78 & 121.11 & -0.535 & 0.039 & -0.606 & 0.029 & -0.757 & 0.054 & -0.637 & 0.045 & 4 & D & 445.4 & $\mathrm{C}$ & 445.4 \\
\hline 579 & TTN012 & 22.77 & 121.13 & -0.545 & 0.188 & -0.179 & 0.092 & -0.445 & 0.119 & -0.124 & & 11 & $\mathrm{D}$ & & 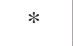 & 488.9 \\
\hline 580 & TTN013 & 22.77 & 121.13 & -0.805 & 0.114 & -0.332 & 0.073 & -0.805 & 0.129 & -0.317 & 0.024 & 6 & D & $* * * * *$ & * & 691.3 \\
\hline 581 & TTN014 & 23.10 & 121.37 & -0.370 & 0.154 & -0.440 & 0.153 & -0.393 & 0.158 & & 0.127 & 16 & $\mathrm{D}$ & 539.6 & $\mathrm{C}$ & 539.6 \\
\hline 582 & & & & & & & & & & & & 15 & D & & . & 1.7 \\
\hline 583 & TTN016 & 22.36 & 120.90 & 0.154 & 0.056 & 0.285 & 0.036 & -0.034 & 0.227 & 0.077 & 0.031 & 2 & B & 842.8 & B & 842.8 \\
\hline 584 & TTN017 & 22.04 & 121.55 & 0.872 & 0.000 & 0.202 & 0.000 & 0.545 & 0.000 & 0.169 & 0.000 & 1 & $\mathrm{C}$ & & $*$ & 288.1 \\
\hline 585 & & 22.82 & & & & & & & & & & 14 & B & & $*$ & \\
\hline 586 & TTN020 & 23.13 & 121.21 & -0.694 & 0.290 & -0.454 & 0.263 & -0.709 & 0.249 & -0.459 & 0.260 & 17 & $\mathrm{D}$ & $* * * * *$ & $*$ & 893.2 \\
\hline 587 & TTN021 & 23.10 & 121.17 & 0.042 & 0.127 & -0.528 & 0.402 & 0.093 & 0.331 & -0 . & 26 & 5 & B & & $*$ & 885.0 \\
\hline 588 & TTN022 & 23.10 & 121.21 & & & & & & & & & 18 & D & $* * * * *$ & * & 442.6 \\
\hline 589 & TTN023 & 23.05 & 121.16 & -0.038 & 0.362 & 0.136 & 0.240 & -0.015 & 0.263 & 0.128 & 0.207 & 13 & D & 542.2 & $\mathrm{C}$ & 542.2 \\
\hline 590 & TTN024 & 22.97 & 121.11 & -0.356 & 0.274 & -0.748 & 0.266 & -0.329 & 0.199 & -0.731 & 0.238 & 19 & B & $* * * * *$ & $*$ & 1458.4 \\
\hline 591 & TTN025 & 22.90 & 121.07 & -0.329 & 0.292 & -0.683 & 0.311 & -0.289 & 0.161 & -0.668 & & 19 & D & 701.1 & $\mathrm{C}$ & 701.1 \\
\hline 592 & TTN026 & 22.86 & 121.08 & -0.441 & 0.247 & -0.524 & 0.191 & -0.400 & 0.094 & -0.520 & 0.115 & 17 & B & $* * * * *$ & $*$ & 996.6 \\
\hline 593 & & 22.81 & & & & & & & & & & 19 & 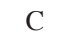 & & $*$ & \\
\hline 594 & TTN028 & 22.78 & 121.06 & -0.627 & 0.264 & -0.646 & 0.219 & -0.613 & 0.248 & -0.657 & 0.207 & 14 & B & 619.8 & $\mathrm{C}$ & 619.8 \\
\hline 595 & TTN029 & 22.72 & 121.04 & 0.025 & 0.581 & 0.056 & 1.100 & -0.028 & 1.049 & -0.021 & 1.252 & 6 & D & $* * * * *$ & $*$ & 405.6 \\
\hline 596 & & 22.70 & & & & & & & & & & 5 & 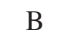 & & $\mathrm{C}$ & \\
\hline 597 & TTN031 & 23.36 & 121.46 & -0.460 & 0.183 & -0.586 & 0 . & -0.432 & 0.144 & -0. & & 15 & D & 512.7 & $\mathrm{C}$ & 512.7 \\
\hline 598 & TTN032 & 23.25 & 121.41 & -0.544 & 0.480 & -0.509 & 0.207 & -0.559 & 0.414 & -0.514 & 0.206 & 17 & D & 736.1 & $\mathrm{C}$ & 736.1 \\
\hline 599 & TTN033 & 23.19 & & & & & & & & & & 14 & D & & $\mathrm{C}$ & \\
\hline 600 & TTN034 & 22.93 & 121.26 & 0.209 & 0.601 & 0.048 & 0.198 & 0.513 & 0.465 & 0.184 & 0.3 & 3 & D & 457.5 & $\mathrm{C}$ & 457.5 \\
\hline 601 & TTN035 & 22.88 & 121.22 & -0.275 & 0.713 & -0.376 & 0.386 & -0.187 & 0.604 & -0.322 & 0.510 & 7 & D & $* * * * *$ & $*$ & 697.6 \\
\hline 602 & TTN036 & 22.80 & 121.19 & -0.928 & 0.222 & -0.443 & 0.207 & -0.889 & 0.151 & -0.435 & 0.222 & 15 & $\mathrm{C}$ & $* * * * *$ & $*$ & 855.1 \\
\hline 603 & TTN037 & 22.53 & 120.84 & -0.122 & 1.225 & -0.219 & 1.453 & 0.005 & 1.356 & -0.158 & 1.182 & 4 & B & 738.2 & $\mathrm{C}$ & 738.2 \\
\hline 604 & TTN038 & 22.46 & 120.93 & -0.462 & 0.000 & -1.021 & 0.000 & -0.794 & 0.000 & -1.024 & 0.000 & 1 & $\mathrm{D}$ & $* * * * *$ & * & 2472.6 \\
\hline 605 & TTN040 & 23.15 & 121.20 & -0.781 & 0.330 & -0.936 & 0.229 & -0.809 & 0.165 & -0.947 & & 13 & B & 726.6 & $\mathrm{C}$ & 726.6 \\
\hline 606 & TTN041 & 23.13 & 121.12 & 0.464 & 0.310 & -0.124 & 0.481 & 0.417 & 0.217 & -0.158 & 0.424 & 15 & B & 431.6 & $\mathrm{C}$ & 431.6 \\
\hline 607 & TTN042 & 23.00 & & & & & & & & & & 12 & D & & B & 824.5 \\
\hline 608 & TTN043 & 23.03 & 121.32 & -0.503 & 0.329 & -0.711 & 0.047 & -0.440 & 0.032 & -0.721 & 0.051 & 2 & D & 490.6 & $\mathrm{C}$ & 490.6 \\
\hline 609 & TTN044 & 23.01 & 121.17 & -0.179 & 0.230 & 0.033 & 0.167 & -0.223 & 0.166 & 0.000 & 0.101 & 14 & $\mathrm{C}$ & $* * * * *$ & $*$ & 391.0 \\
\hline 610 & TTN045 & 22.98 & & & & & & & & & & 9 & D & & $\mathrm{C}$ & 539.5 \\
\hline 611 & TTN046 & 22.96 & 121.23 & -0.129 & 0.233 & -0.346 & 0.173 & -0.173 & 0.128 & -0.379 & 0.117 & 14 & $\mathrm{C}$ & 529.1 & $\mathrm{C}$ & 529.1 \\
\hline 612 & TTN047 & 22.84 & 121.13 & -0.788 & 0.189 & -0.998 & 0.126 & -0.995 & 0.099 & -1.032 & 0.127 & 2 & $\mathrm{C}$ & $* * * * *$ & $*$ & 2508.5 \\
\hline 613 & TTN048 & 22.79 & 121.08 & -0.502 & 0.164 & -0.204 & 0.146 & -0.547 & 0.065 & -0.238 & 0.118 & 9 & D & $* * * * *$ & $*$ & 599.7 \\
\hline 614 & TTN049 & 22.73 & 121.10 & -0.767 & 0.219 & -0.290 & 0.016 & -0.833 & 0.012 & -0.308 & 0.018 & 3 & D & $* * * * *$ & $*$ & 680.9 \\
\hline 615 & TTN050 & 22.67 & 121.03 & -0.296 & 0.229 & -0.590 & 0.192 & -0.342 & 0.134 & -0.613 & 0.085 & 11 & $\mathrm{D}$ & $* * * * *$ & $*$ & 1179.1 \\
\hline 616 & TTN051 & 23.19 & 121.02 & -0.376 & 0.377 & -0.797 & 0.391 & -0.420 & 0.337 & -0.818 & 0.324 & 14 & B & $* * * * *$ & $*$ & 1704.7 \\
\hline 617 & TTN052 & 22.60 & 120.95 & -0.534 & 0.079 & -0.698 & 0.199 & -0.857 & 0.109 & -0.883 & 0.112 & 3 & B & $* * * * *$ & $*$ & 1916.8 \\
\hline
\end{tabular}




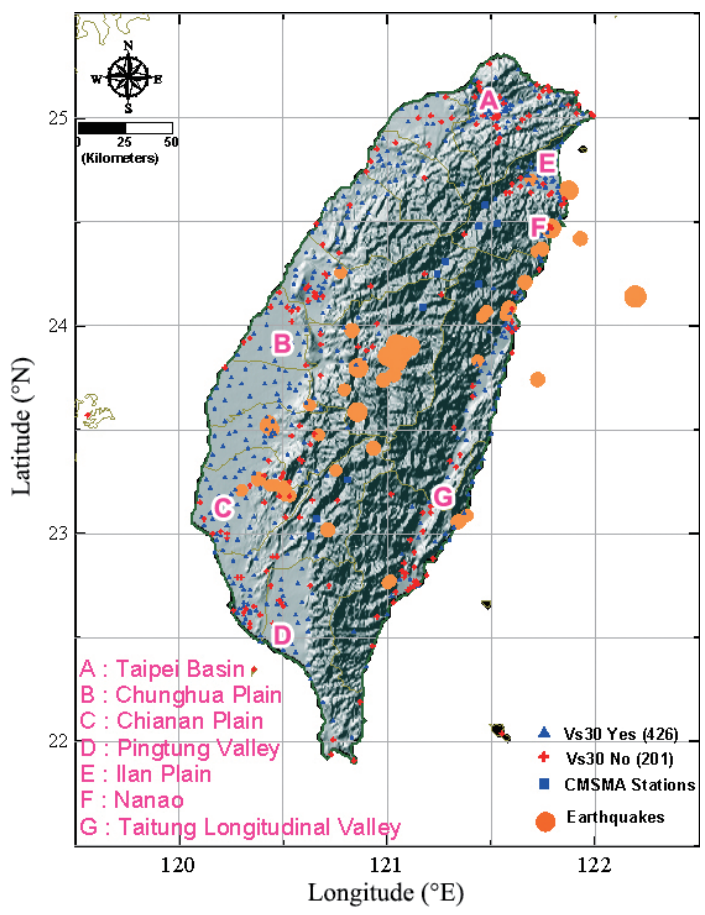

Fig. 1. Distribution of the earthquakes, Taiwan Strong Motion Instrumentation Program (TSMIP) and Central Mountain Strong Motion Array (CMSMA) free-field stations used in this study. Relevant localities and topography are also indicated.

Table 2. Station code, location, site classifications, ground motion total and intra-event residuals, measured and estimated Vs30 of the CMSMA stations.

\begin{tabular}{|c|c|c|c|c|c|c|c|c|c|c|c|c|c|c|c|c|}
\hline \multirow{2}{*}{ No. } & \multirow{2}{*}{ Stname } & \multirow{2}{*}{$\begin{array}{l}\text { Lat } \\
\left({ }^{\circ} \mathbf{N}\right)\end{array}$} & \multirow{2}{*}{$\begin{array}{c}\text { Long } \\
\left({ }^{\circ} \mathbf{E}\right)\end{array}$} & \multicolumn{4}{|c|}{ TotalRes } & \multicolumn{4}{|c|}{ IntraRes } & \multirow{2}{*}{$\mathbf{R}$} & \multirow{2}{*}{ Site $\dagger$} & \multirow{2}{*}{$\begin{array}{c}\text { Vs30 } \\
\left(\mathrm{m} \mathrm{s}^{-1}\right)\end{array}$} & \multirow{2}{*}{ Site } & \multirow{2}{*}{$\begin{array}{l}\text { Vs30N } \\
\left(\mathrm{m} \mathrm{s}^{-1}\right)\end{array}$} \\
\hline & & & & \multicolumn{2}{|c|}{ PGAh \pm sd } & \multicolumn{2}{|c|}{ PGVh \pm sd } & \multicolumn{2}{|c|}{ PGAh \pm sd } & \multicolumn{2}{|c|}{ PGVh \pm sd } & & & & & \\
\hline 1 & CMA001 & 22.99 & 120.63 & -0.336 & 0.214 & -0.466 & 0.116 & -0.316 & 0.131 & -0.500 & 0.046 & 11 & $\mathrm{D}$ & $* * * * *$ & $*$ & 961.3 \\
\hline 2 & CMA002 & 23.07 & 120.66 & -0.481 & 0.170 & -0.653 & 0.257 & -0.461 & 0.056 & -0.687 & 0.137 & 11 & $\mathrm{D}$ & $* * * * *$ & $*$ & 1346.3 \\
\hline 3 & CMA004 & 23.26 & 120.81 & 0.035 & 0.239 & -0.792 & 0.156 & 0.412 & 0.230 & -0.5 & 0.096 & 5 & B & $* * * * *$ & $*$ & 1149.7 \\
\hline 4 & CMA008 & 24.20 & 121.44 & -0.617 & 0.241 & -0.991 & 0.225 & -0.541 & 0.342 & -0.934 & 0.289 & 7 & $\mathrm{D}$ & $* * * * *$ & $*$ & 2103.9 \\
\hline 5 & CMA012 & 24.09 & 121.17 & -0.193 & 0.658 & 0.041 & 0.708 & -0.062 & 0.741 & 0.134 & 0.792 & 6 & B & & $*$ & 307.2 \\
\hline 6 & CMA014 & 24.58 & 121.47 & -0.324 & 0.345 & -0.763 & 0. & -0.285 & 0.389 & -0.747 & 0.3 & 8 & B & $* * * * *$ & $*$ & 1500.2 \\
\hline 7 & CMA015 & 24.49 & 121.53 & -0.360 & 0.309 & -0.645 & 0.192 & -0.280 & 0.379 & -0.632 & 0.283 & 9 & B & $* * * * *$ & * & 1219.2 \\
\hline 8 & $\mathrm{CM}$ & 24.48 & 121.44 & -0.696 & 0.255 & -0.898 & & -0.623 & 0.603 & -0.857 & 23 & 7 & B & & $*$ & 1829.1 \\
\hline 9 & CMA018 & 24.31 & 121.28 & -0.262 & 0.258 & -1.409 & 0.060 & -0.081 & 0.035 & -1.238 & 0.104 & 3 & B & $* * * * *$ & * & 3637.8 \\
\hline 10 & CMA019 & 24.25 & 121.24 & 0.549 & 0.000 & -0.262 & 0.000 & 0.687 & 0.000 & -0.017 & 0.000 & 1 & B & $* * * * *$ & * & 402.9 \\
\hline
\end{tabular}

Note: (1) STname = Station Name.

(2) Lat = Station Latitude.

(3) Long = Station Longitude.

(4) TotalRes $=$ Total Residual in ln unit.

(5) IntraRes= Intra-event Residual in In unit.

(6) $P G A h=$ The mean of residual for horizontal component of peak ground acceleration.

(7) $P G V h=$ The mean of residual for horizontal component of peak ground velocity.

(8) $s d=$ standard deviation.

(9) $R=$ Number of Records.

(10) Vs30 = The average shear-wave velocity in the upper $30 \mathrm{~m}$ of sediments in $\mathrm{m} \mathrm{s}^{-1}$.

(11) Vs30N = The estimated Vs30 values from the relation between the intra-event residual and the Vs30 in $\mathrm{m} \mathrm{s}^{-1}$.

$\dagger$ Determined by Lee et al. (2001), on the basis of surface geology and borehole data.

$\neq$ Determined according to UBC1997. 


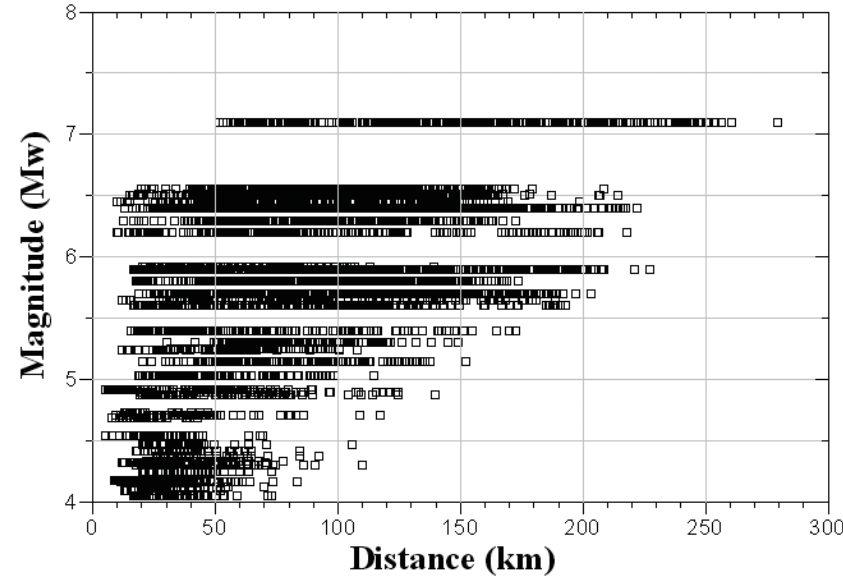

Fig. 2. Magnitude-distance distribution of the earthquakes used in this study.

magnitude, $\mathrm{a}$ is the geometric spreading coefficient, $\mathrm{b}$ is the inelastic attenuation coefficient, $\mathrm{c}$ is magnitude coefficient, $\mathrm{d}$ is a constant, $\mathrm{h}_{1}$ and $\mathrm{h}_{2}$ are close-in distance saturation coefficients. Vref is the reference shear-wave velocity. The coefficients a, b, c, d, d', e, $h_{1}, h_{2}$, and Vref are to be determined by regression from the data. In Eq. (2) the constant changed from $d$ to $d^{\prime}$ while we specify Vref, the reference velocity to be $760 \mathrm{~m} \mathrm{~s}^{-1}$. In this case the soil amplifications are specified relative to motions that would be recorded on a NEHRP B/C boundary site condition.

The coefficients in the equation for predicting ground motion were determined using a two-stage regression procedure. The least square method was used in the regression. A similar approach was used previously by Joyner and Boore (1993) and Liu and Tsai (2005).

The two models are adopted to account for different situations. Model 1, uses recordings from all 627 strongmotion sites, is especially useful for early warning systems to make quick assessment and timely reporting of the PGA and PGV maps. Their results will be critical for effective emergency response operations. Model 2, using 426 strongmotion sites to incorporate a site effect term with available Vs30, is aimed to reduce the standard deviation of the predicted ground motion. This approach emphasizes direct use of strong ground motion recordings for seismic ground shaking estimation for engineering applications.

The residual value, i.e., site response factor, is defined as the difference between logarithms of the observed and the predicted ground motion, and is expressed by the following equation:

$r=\ln Y_{s}-\ln Y_{r}=\eta+\varepsilon$

where $Y_{\mathrm{s}}$ is the observed value, $\mathrm{Y}_{\mathrm{r}}$ is the predicted value from Eq. (1), $\eta$ is the earthquake inter-event errors with standard deviation equal to $\tau$, and $\varepsilon$ is intra-event errors with standard deviation equal to $\sigma$. The $\eta$ and $\varepsilon$ are assumed to be normally distributed independent variants with variances $\tau^{2}$ and $\sigma^{2}$. The amplification factors of site effect can be calculated from exponent of (r). The standard deviation of total residual $\sigma_{\mathrm{T}}$ is given by the equation:

$\sigma_{\mathrm{T}}=\sqrt{\sigma^{2}+\tau^{2}}$

The residuals due to regression were decomposed into interevent (earthquake-to-earthquake) and intra-event residuals. The inter-event and intra-event residuals are calculated using Eqs. (3.15) and (3.16) in Campbell and Bozorgnia (2007).

We analyzed the relations between the intra-event residuals $\varepsilon$ and the average shear-wave velocity in the upper $30 \mathrm{~m}$ of sediments, Vs30 using the following equation (Boore et al. 1997; Choi and Stewart 2005; Liu et al. 2013):

$\varepsilon=\mathrm{f} \ln (\mathrm{Vs} 30 / \mathrm{Vref})$

where the coefficient $\mathrm{f}$ and Vref are to be determined by regression from the data.

\section{RESULTS AND DISCUSSION}

\subsection{Attenuation Relationships for Vertical and Horizontal PGA and PGV}

Ground motion characteristic studies in Taiwan require ground-motion attenuation models. Attenuation relationships, or "GMPEs", provide an efficient means for predicting the level of ground shaking and its associated uncertainty at any given site or location, as well as for use in seismic hazard analyses (Bolt and Abrahamson 2003). An attenuation relationship is a mathematical equation that relates a specific strong-motion ground shaking parameter to a number of earthquake seismological parameters and the recording site. The seismological parameters quantitatively characterize the earthquake source, the wave propagation path between the source and the site, and the soil and geological profile beneath the site (Campbell 2003).

Regressions on the dataset for Model 1 without differentiating site conditions and Model 2 with site conditions have resulted in the coefficients of the attenuation relationships, as given in Tables 3 and 4, respectively, for the vertical and horizontal components of PGA and PGV in Taiwan area. In Tables 3 and $4, \sigma_{1}$ and $\sigma_{2}$ are standard deviations on $\ln (\mathrm{PGA}, \mathrm{PGV})$.

\subsection{Analyses of Site Total Residuals for PGA and PGV}

Residual examination for sites with qualitative soil categories is a useful method for sets of records where site 
information is not complete, and hence cannot be included explicitly within the equation (Abrahamson and Litehiser 1989). In this study we analyze the residuals to investigate variations in PGA and PGV with respect to site conditions.

The mean and standard deviation of total and intra-event residuals for 627 stations for the vertical and horizontal PGA and PGV are given in columns $5-8$ of Tables 1 and 2 . The corresponding contour maps of mean of total residuals for the horizontal PGA and PGV are shown in the Figs. 3 and 4, respectively. The total residual patterns, especially those for PGV, agree reasonably well with the regional geology and topography patterns. Notably, the PGV residual is more

Table 3. Coefficients for the vertical and horizontal components of PGA and PGV from Eq. (1).

\begin{tabular}{c|ccccccccc}
\hline PGA & $\mathbf{a}$ & $\mathbf{b}$ & $\mathbf{c}$ & $\mathbf{d}$ & $\mathbf{h}_{\mathbf{1}}$ & $\mathbf{h}_{\mathbf{2}}$ & $\mathbf{e}$ & Vref & $\boldsymbol{\sigma}_{\mathbf{1}}$ \\
\hline V-Comp. & -1.340 & -0.0036 & 1.101 & 1.824 & 1.62 & 0.0 & & 0.640 \\
H-Comp. & -0.852 & -0.0071 & 1.027 & 1.164 & 1.24 & 0.0 & & 0.683 \\
\hline PGV & $\mathbf{a}$ & $\mathbf{b}$ & $\mathbf{c}$ & $\mathbf{d}$ & $\mathbf{h}_{\mathbf{1}}$ & $\mathbf{h}_{\mathbf{2}}$ & $\mathbf{e}$ & Vref & $\boldsymbol{\sigma}_{\mathbf{1}}$ \\
\hline V-Comp. & -0.953 & -0.0012 & 1.534 & -5.184 & 1.19 & 0.0 & & 0.566 \\
H-Comp. & -0.857 & -0.0023 & 1.486 & -4.371 & 1.34 & 0.0 & & 0.663 \\
\hline
\end{tabular}

Table 4. Coefficients for the vertical and horizontal components of PGA and PGV from Eqs. (2) and (3).

\begin{tabular}{ccccccccccc}
\hline PGA & $\mathbf{a}$ & $\mathbf{b}$ & $\mathbf{c}$ & $\mathbf{d}$ & $\mathbf{h}_{\mathbf{1}}$ & $\mathbf{h}_{\mathbf{2}}$ & $\mathbf{d}^{\prime}$ & $\mathbf{e}$ & Vref & $\boldsymbol{\sigma}_{2}$ \\
\hline V-Comp. & -1.340 & -0.0036 & 1.101 & 1.824 & 1.62 & 0.0 & 1.635 & -0.263 & 370 & 0.621 \\
H-Comp. & -0.852 & -0.0071 & 1.027 & 1.164 & 1.24 & 0.0 & 0.886 & -0.375 & 362 & 0.647 \\
\hline PGV & $\mathbf{a}$ & $\mathbf{b}$ & $\mathbf{c}$ & $\mathbf{d}$ & $\mathbf{h}_{\mathbf{1}}$ & $\mathbf{h}_{\mathbf{2}}$ & $\mathbf{d}^{\prime}$ & $\mathbf{e}$ & Vref & $\boldsymbol{\sigma}_{2}$ \\
\hline V-Comp. & -0.953 & -0.0012 & 1.534 & -5.184 & 1.19 & 0.0 & -5.351 & -0.230 & 368 & 0.549 \\
H-Comp. & -0.857 & -0.0023 & 1.486 & -4.371 & 1.34 & 0.0 & -4.760 & -0.549 & 374 & 0.587 \\
\hline
\end{tabular}

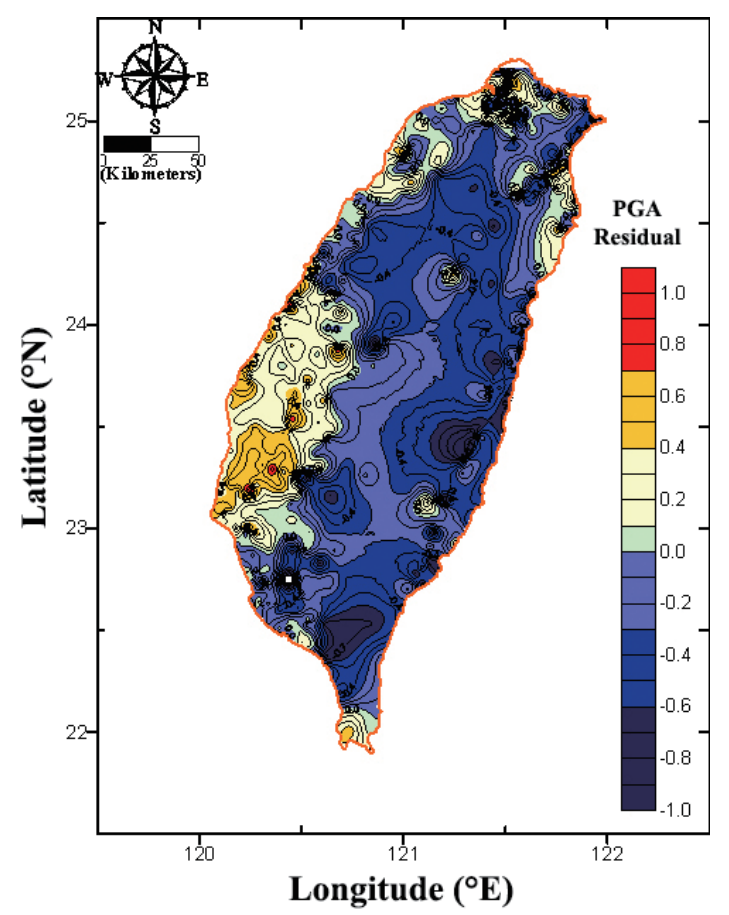

Fig. 3. The total residual contour map (in ln unit) for horizontal PGA.

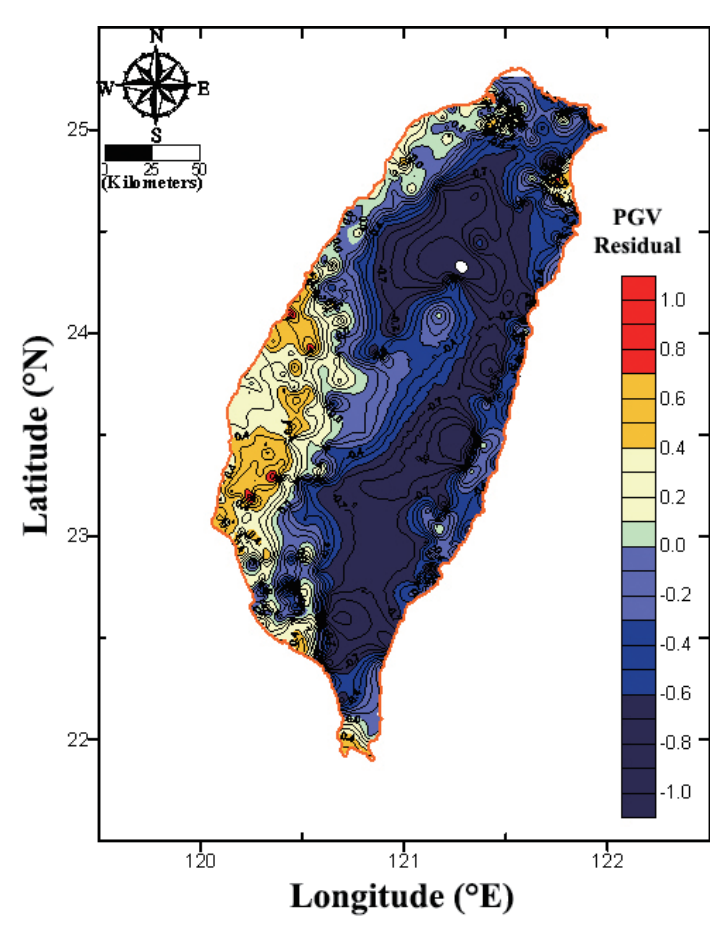

Fig. 4. The total residual contour map (in ln unit) for horizontal PGV. 
sensitive to the site class than the PGA residual, because the PGA is a high-frequency parameter which is less affected by local site conditions. Local site conditions can dramatically affect the strong motions recorded (Douglas 2003). Furthermore, in order to understand the relation between the ground motion parameter site residuals and Vs30, we plot the Vs30 contour map based on 426 measured data as shown in Fig. 5 . We also found that the PGV residual contour patterns in major plain areas are similar to the Vs30 contour patterns.

The purpose of Model 1 is early warning systems to make quick assessment and timely reporting of the PGA and PGV maps. Hence, the equation form is so simple that is does not include the fault type (focal mechanism), intra- or inter-event term and site effect term such as Vs30. Their results will be critical for effective emergency response operations. Accordingly, following a disastrous earthquake, quick assessment and timely reporting of PGA and PGV maps will be critical for effective emergency response operations. Thus, after an earthquake we can combine the simple attenuation relationships, as determined from Eq. (1), and the total residuals, as determined from Table 1, to provide near realtime estimation and reporting of the PGA and PGV values for the Taiwan area.

\subsection{Comparisons of Model Predictions Including Vs30}

In early times most attenuation relationships used broad site categories such as "rock", "stiff-soil", and "softsoil". There has recently been a move toward using quantitative site classifications based on the shear-wave velocity measured at the strong-motion site. The most commonly used parameter is the average shear-wave velocity over the top $30 \mathrm{~m}$ (Vs30) (Boore et al. 1997; Bolt and Abrahamson 2003). For ground-motion model development, developers systematically evaluated a list of predictor parameters to consider for predicting earthquake shakings. One of the most significant decisions made by all developers was to use the average shear-wave velocity in the upper $30 \mathrm{~m}$ of sediments, Vs30, as the parameter for characterizing soilstiffness effects on ground motions (Power et al. 2008).

A major limitation in using quantitative site descriptions is that the Vs30 information is not available for most strong-motion recording sites. This situation has been greatly improved for our study because measured shear-wave velocity profiles are available at 439 strong-motion recording sites in the Taiwan area. Of these, 426 stations were used in this study as given in Table 1 . To incorporate a site effect term based on the average shear-wave velocity over the top $30 \mathrm{~m}$ (Vs30) into the attenuation relationship, the analytical form used in this study is given in Eq. (2).

As our understanding and modeling of attenuation relationships improve, there will be a trend toward reducing the modeling variability. In empirical attenuation models the modeling variability given for the model is the standard deviation (Bolt and Abrahamson 2003). In addition to the median ground motion, the standard deviation of the ground motion is also important for seismic hazard analyses. After incorporating a site effect term, Vs30 in the attenuation relationships, the standard deviations between the observed and predicted values are reduced from $0.683-0.647$ for horizontal PGA, and from 0.663 - 0.587 for horizontal PGV, respectively. We found only minor reduction in standard deviation for PGA. In contrast, the PGV standard deviation is significantly reduced by about $11 \%$.

We analyzed the site effect term using the amplification factor (relative to a site with Vs30 $=760 \mathrm{~m} \mathrm{~s}^{-1}$ ): $\exp [-e \ln (760 /$ Vref $)+$ Res_av], where Res_av is the average intra-event residual from Eq. (1) for each site and the e and Vref of horizontal PGA and PGV are given in Table 4. The corrected site amplification factor contour maps relative to a NEHRP B/C boundary site condition (Vs30 $=760 \mathrm{~m} \mathrm{~s}^{-1}$ ) are plotted in Fig. 6 for horizontal PGA and in Fig. 7 for horizontal PGV, respectively. The amplification factors are contoured at 0.2 intervals. They range from $3.4-0.4$ for horizontal PGA and from 4.0 - 0.4 for horizontal PGV, respectively. Both total residual and amplification factor contour maps of horizontal PGV have similar patterns, revealing that the Changhua Plain, Chianan Plain, Pingtung Valley, Ilan Plain, and Taipei Basin have high values, implying large amplification of ground motions.

\subsection{Construction of a Refined Vs30 Map}

Correlations between the intra-event residual and

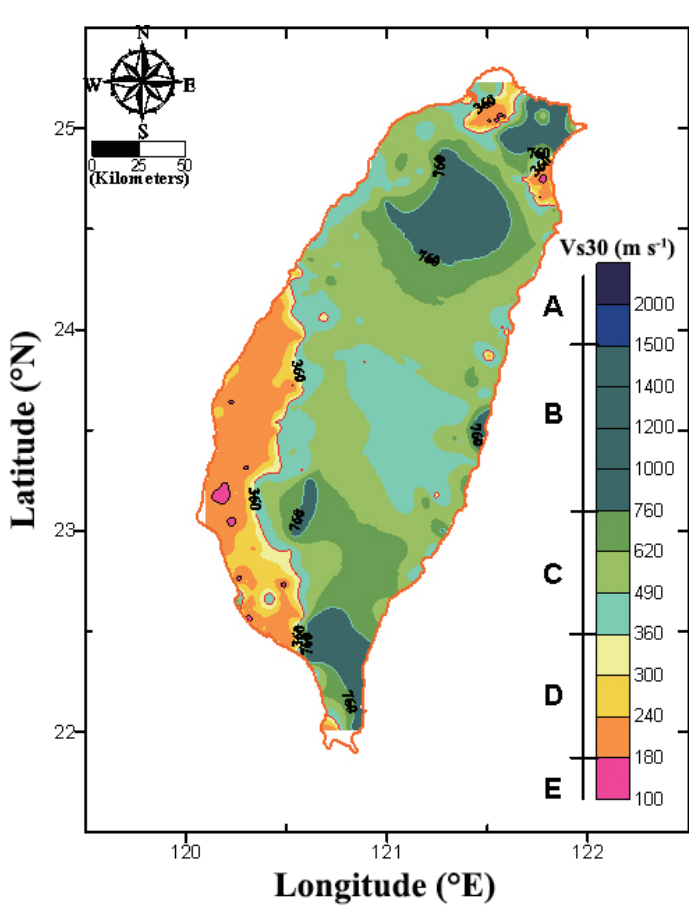

Fig. 5. The Vs30 contour map based on measured data. 


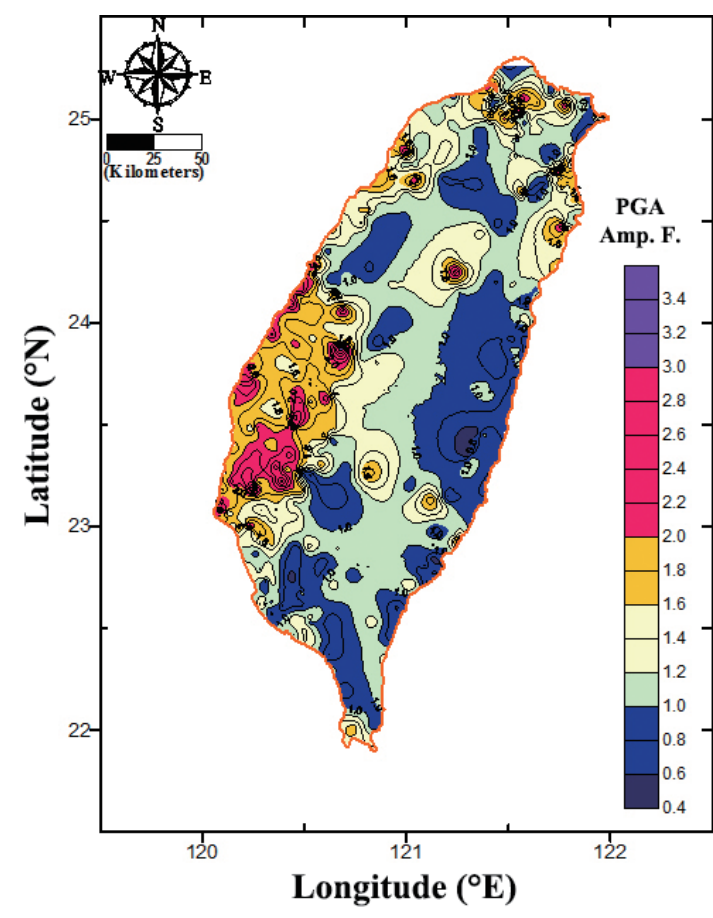

Fig. 6.The corrected site amplification factor contour map relative to a NEHRP B/C boundary site condition (Vs30 = $760 \mathrm{~m} \mathrm{~s}^{-1}$ ) for horizontal PGA. average shear-wave velocity in the upper $30 \mathrm{~m}$ of sediments Vs30 are plotted in Figs. 8 and 9 for vertical and horizontal PGA and Figs. 10 and 11 for vertical and horizontal PGV, respectively. The regression equations and the coefficient of determination $\mathrm{R}^{2}$ are also shown in the figures. $\mathrm{R}$ is the correlation coefficient used to measure how well the linear relationship exists between the values for the two variables. The corresponding coefficients ( $\mathrm{f}$ and Vref) are given in Table 5. Reading from Fig. 11 the value of $R$ was $0.712\left(R^{2}=0.5070\right)$ indicating that the horizontal PGV residuals are much more dependent on Vs30 than the residuals for the other three ground motion parameters, probably due to their frequency content which is less affected by local site conditions.

Since the Vs30 information is not available for a significant number of TSMIP and CMSMA stations, especially for those having a NEHRP B and C site conditions, here we use the relations between the intra-event site residual and the Vs30 for horizontal PGV, as given by Eq. (6), to estimate the Vs30 for these stations that have recorded strong motions. The estimated Vs30 values, denoted as Vs30N, are given in the last column of Tables 1 and 2. A refined Vs30 contour map that includes the $201 \mathrm{Vs} 30 \mathrm{~N}$ sites and $426 \mathrm{Vs} 30$ sites is shown in Fig. 12. By comparing the Vs30 contour maps, as shown in Fig. 5 based on 426 Vs30 sites with Fig. 12, we can find the following results: (1) Fig. 12 offers a more detailed Vs30 contour map for using data from more Vs30 sites. (2) The class D contour pattern is similar. (3) The class E area appears in the Changhua Plain in Fig. 12 needed to pay more

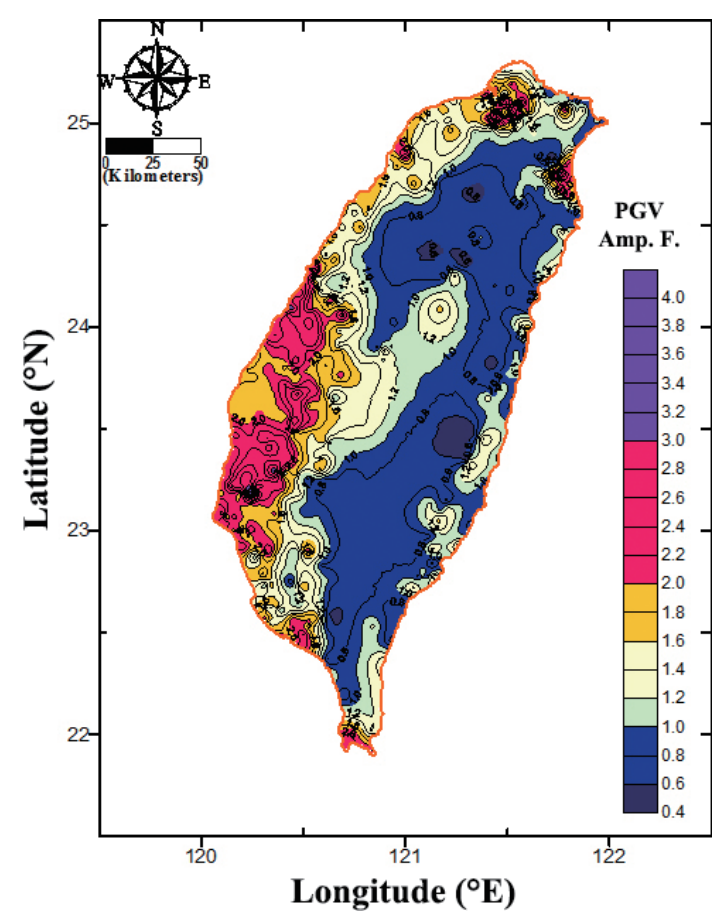

Fig. 7. The corrected site amplification factor contour map relative to a NEHRP B/C boundary site condition $(\mathrm{Vs} 30=$ $760 \mathrm{~m} \mathrm{~s}^{-1}$ ) for horizontal PGV.

attention. (4) The rocky areas of classes B and A coincide with the foothill and mountain areas distribution in Fig. 12.

We compared our refined Vs30 results with studies by the United States Geological Survey (USGS) (http://earthquake.usgs.gov/hazards/apps/vs30/predefined.php) and Lee and Tsai (2008). The refined Vs30 pattern in this study is similar to that in USGS. Previously, Lee and Tsai (2008) mapped the Vs30 distribution in Taiwan using $230 \mathrm{P}$ and $\mathrm{S}$ wave velocity (PS) logging at soil and soft rock strong-motion station sites and 4885 engineering boreholes. The Vs30 map supplied important knowledge for each strong-motion station, and for sites between stations in Taiwan. However, the accuracy of such mapping is inevitably dependent on the amount and quality of data. In eastern Taiwan and the southern tip of Taiwan, where boreholes are few and scattered, the map accuracy is relatively poor. The mapping results use the 2000 - 2005 Vs measurements and may be further refined based on new data. Kuo et al. (2012) reclassified the 439 drilled free-field TSMIP stations and compared the results with that of Lee and Tsai (2008). It was found that 80 of the 436 stations in common were misclassified by Lee and Tsai (2008). It is probably that Lee and Tsai (2008) focused on mapping the soil sites, and thus tentatively assigned all of the unmeasured rock site stations a value of $760 \mathrm{~m} \mathrm{~s}^{-1}$.

We compared the Vs30 estimations from Lee and Tsai (2008) with the Vs30N from this study. The above relation is plotted in Fig. 13. The regression equations and the coefficient of determination $\mathrm{R}^{2}$ are also shown in the figures. $\mathrm{R}$ is 


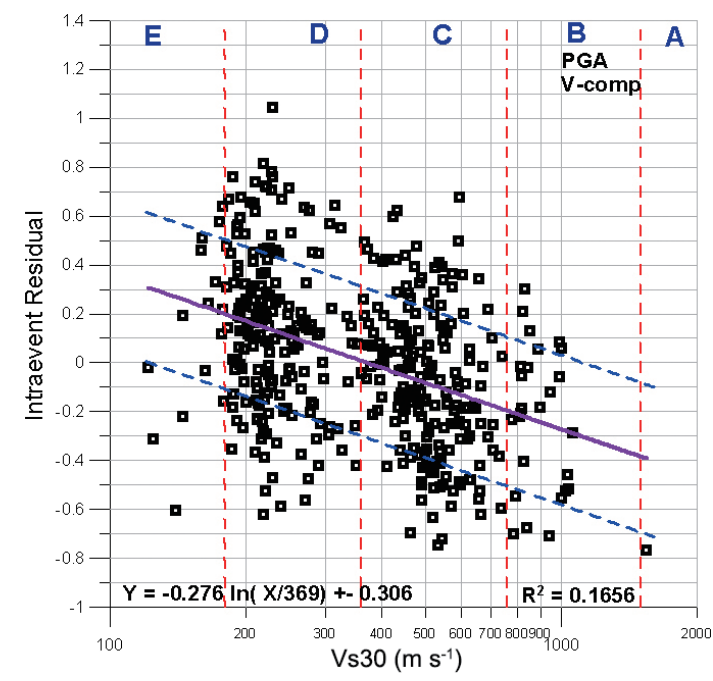

Fig. 8. The relation between the intra-event residual of vertical PGA and the average shear-wave velocity in the upper $30 \mathrm{~m}$ of sediments Vs30.

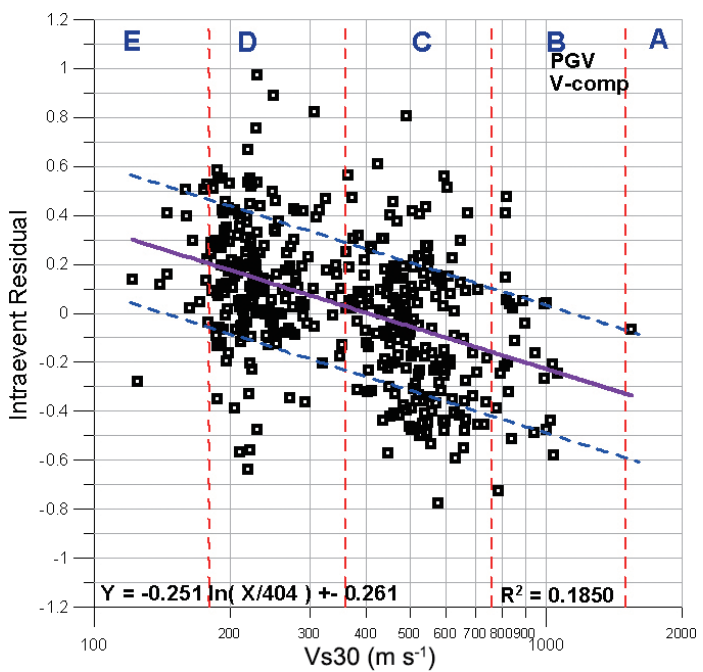

Fig. 10. The relations between the intra-event residual of vertical PGV and the average shear-wave velocity in the upper $30 \mathrm{~m}$ of sediments Vs30.

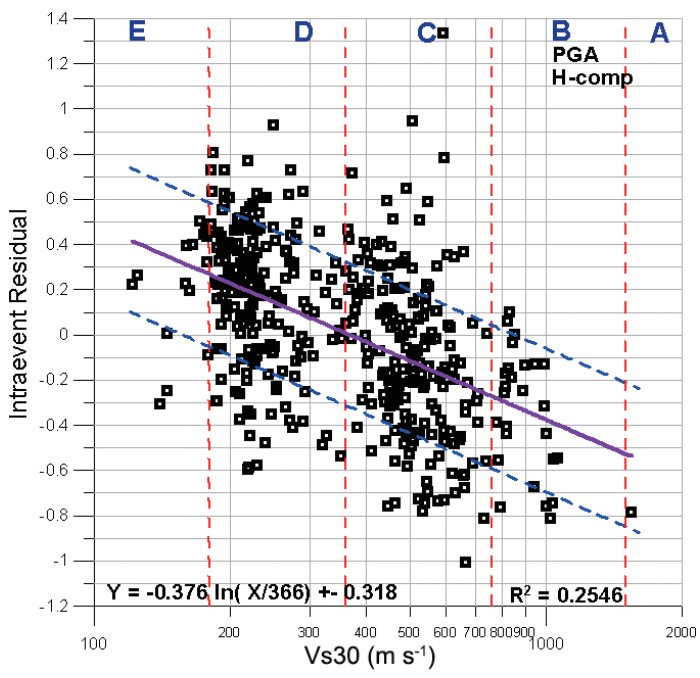

Fig. 9. The relation between the intra-event residual of horizontal PGA and the average shear-wave velocity in the upper $30 \mathrm{~m}$ of sediments Vs30.

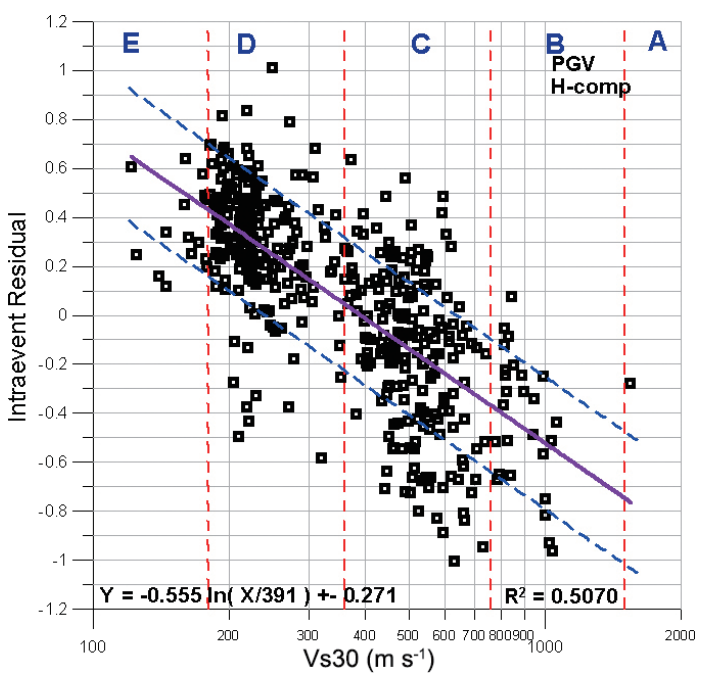

Fig. 11. The relations between the intra-event residual of horizontal PGV and the average shear-wave velocity in the upper $30 \mathrm{~m}$ of sediments Vs30.

Table 5. Coefficients for the vertical and horizontal components of PGA and PGV from Eq. (6).

\begin{tabular}{ccccc}
\hline PGA & $\mathbf{f}$ & Vref & $\boldsymbol{\sigma}$ & $\mathbf{R}^{2}$ \\
\hline V-Comp. & -0.276 & 369 & 0.306 & 0.1656 \\
H-Comp. & -0.376 & 366 & 0.318 & 0.2546 \\
\hline PGV & $\mathbf{f}$ & Vref & $\boldsymbol{\sigma}$ & $\mathbf{R}^{2}$ \\
\hline V-Comp. & -0.251 & 404 & 0.261 & 0.1850 \\
H-Comp. & -0.555 & 391 & 0.271 & 0.5470 \\
\hline
\end{tabular}




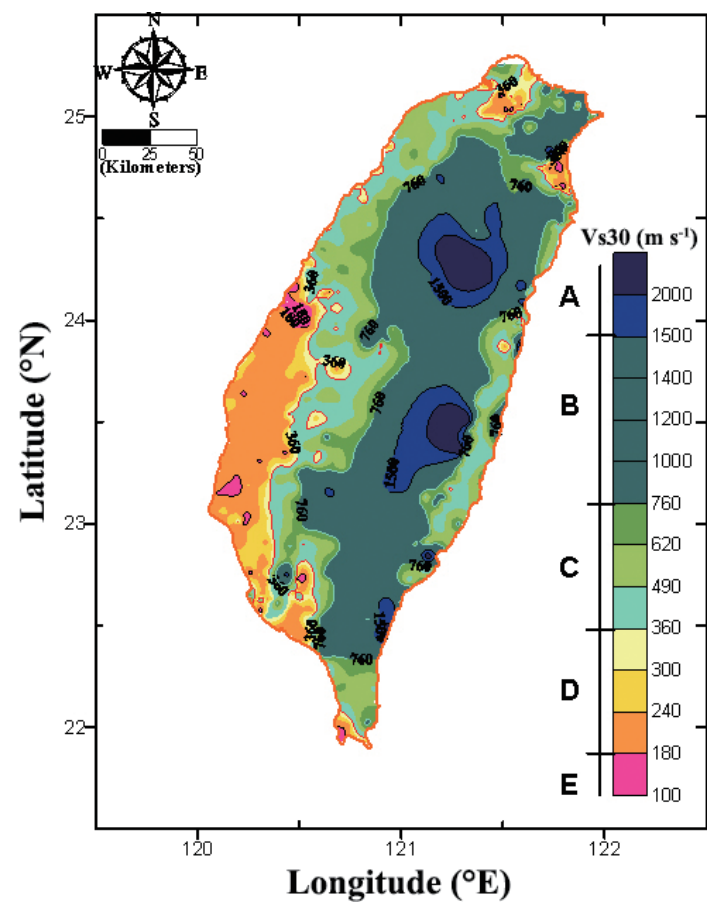

Fig. 12. The refined Vs30 contour map based on combined measured and estimated data.

the correlation coefficient used to measure how good a linear relationship exists between the values for the two variables. The L1 and L2 were conducted for Vs30 estimated from Lee and Tsai (2008) ranging from $110-835$ and $750 \mathrm{~m} \mathrm{~s}^{-1}$, respectively. Reading from Fig. 13, the values of $\mathrm{R}$ are 0.739 $\left(R^{2}=0.549\right)$ and $0.706\left(R^{2}=0.499\right)$ indicating that a highly dependent relation exists between the two estimated Vs30 models. One model is adopted between the Standard penetration test $\mathrm{N}$ value (SPT-N) and the Vs from Lee and Tsai (2008) while the other model is conducted between the horizontal PGV intra-event residuals and Vs30 from this study. The result also shows that residual examination for sites based on accelerographic recordings is a useful method to evaluate the site effects.

Site classifications for 426 of the 439 drilled free-field TSMIP stations were used in this study. There is one Class A site, 26 Class B sites, 194 Class C sites, 189 Class D sites, and 16 Class E sites. Evidently, most drilled stations belong to Classes C and D (89.9\%). This means that most of the drilled stations were installed in metropolitan districts, which are usually located on sediments or soft rocks. The number of surveyed stations in the EGDT reached a total of 469 by 2010. Of these, 439 stations were drilled and the other 30 stations, to which the equipment could not be transported or where the landowner declined the request for drilling (Kuo et al. 2012). A major objective of this study is to estimate the Vs30 values for the 201 stations which were not drilled. Note that 426 drilled Vs30 stations and 7833 high quality strong motion recording were used to estimate Vs30 by in-

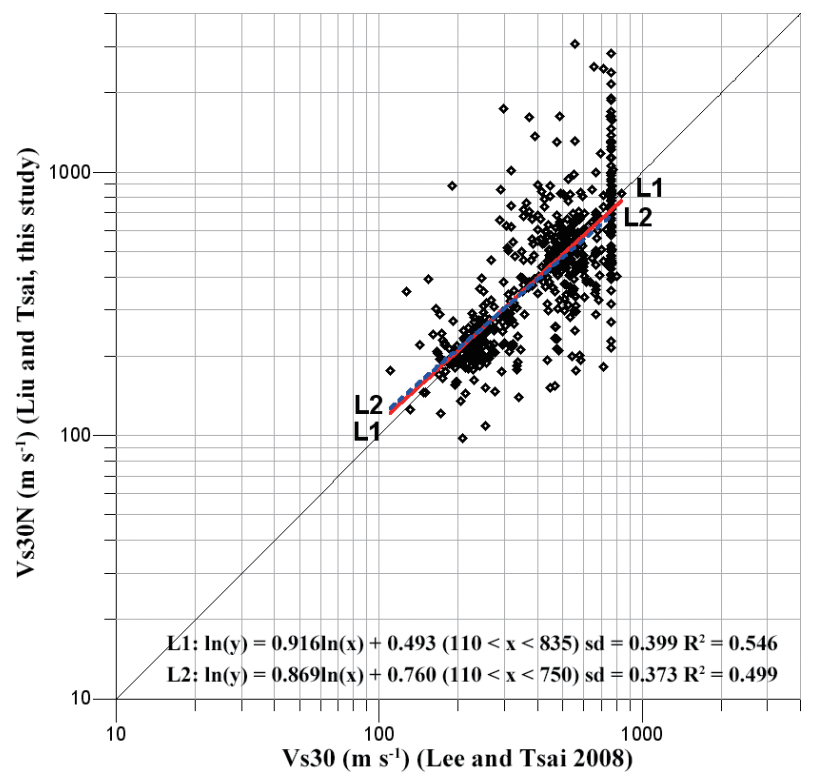

Fig. 13. The relation between the Vs30 estimated from Lee and Tsai (2008) and the Vs30N from this study.

tra-event residual technique. Of the 201 stations, 21 Class A sites, 43 Class B sites, 75 Class C sites, 50 Class D sites, and 12 Class E sites. A large number of undrilled stations belong to Classes A and B (64/201), usually located in foothill and mountain areas. Hence, the refined Vs30 map would be helpful for engineering and other research purposes, and especially applicable to the foothill and mountain areas.

\section{CONCLUSIONS}

From above results and discussion, we can summarize our findings as follows:

(1) The PGV residual contour pattern is highly similar to the Vs30 contour pattern. The horizontal PGV residual as found more dependent on Vs30 than the vertical and horizontal PGA, as well as vertical PGV, probably due to their high-frequency content which is less affected by local site conditions.

(2) By comparing the standard deviations between the observed and predicted ground motion values before and after incorporating the site effect term Vs30, the reduction in standard deviation for PGA is only moderate. In contrast, the PGV standard deviation is significantly reduced by about $11 \%$. Evidently, the refined attenuation relationships will be more appropriate for engineering applications.

(3) After analyzing the local site effect in terms of the amplification factor (relative to a site with Vs30 $=760 \mathrm{~m}$ $\left.\mathrm{s}^{-1}\right)$, the Changhua Plain, Chianan Plain, Pingtung Valley, 
Ilan Plain, and Taipei Basin were revealed to high values, implying large ground motion amplification. Large parts of the Central Mountain Range have low values, implying potential ground motion de-amplification.

(4) Following a disastrous earthquake, quick assessment and timely reporting of PGA and PGV maps will be critical for effective emergency response operations. Thus, after an earthquake, we can combine the simple attenuation relationships, as determined from Eq. (1), and the site residuals, as given in Tables 1 and 2, to provide near real-time estimation and reporting of the PGA and PGV values for the Taiwan area.

(5) Finally, using the correlations between the intra-event residual and Vs30 according to Eq. (6), we can estimate Vs30 for stations that recorded strong motions, but whose Vs30 information is not available. Our approach including sites with estimated Vs30 has resulted in a detailed Vs30 contour map to facilitate more realistic seismic hazard assessment for Taiwan, especially applicable to the foothill and mountain areas.

Acknowledgements We thank the Central Weather Bureau of Taiwan and Institute of Earth Science, Academia Sinica for providing us with the strong motion data. We are also benefitted from the engineering geological database provided by the cooperated projects of the NCREE and the CWB of Taiwan. In addition, we are grateful to the Anonymous Reviewers for their critical and helpful comments which led to significant improvement in the paper. This research was supported by the Taiwan Earthquake Research Center (TEC) funded through the Ministry of Science and Technology of the Republic of China under Grant No. NSC1002116-M-244-001 and MOST104-2216-M-244-001. The TEC contribution number for this article is 00114 .

\section{REFERENCES}

Abrahamson, N. A. and J. J. Litehiser, 1989: Attenuation of vertical peak acceleration. Bull. Seismol. Soc. Am., 79, 549-580.

Anderson, J. G., Y. Lee, Y. Zeng, and S. Day, 1996: Control of strong motion by the upper 30 meters. Bull. Seismol. Soc. Am., 86, 1749-1759.

Bolt, B. A. and N. A. Abrahamson, 2003: Estimation of strong seismic ground motions. In: Lee, W. H. K., H. Kanamori, P. C. Jennings, and C. Kisslinger (Eds.), International Handbook of Earthquake and Engineering Seismology, International Geophysics, Vol. 81, part B, Academic Press, 983-1001, doi: 10.1016/S00746142(03)80173-0. [Link]

Boore, D. M., W. B. Joyner, and T. E. Fumal, 1993: Estimation of response spectra and peak accelerations from Western North American earthquakes: An interim report. U.S. Geological Survey, Open-File Report 93-
$509,72 \mathrm{pp}$.

Boore, D. M., W. B. Joyner, and T. E. Fumal, 1997: Equations for estimating horizontal response spectra and peak acceleration from western North American earthquakes: A summary of recent work. Seismol.Res. Lett., 68, 128-153.

Borcherdt, R. D., 1970: Effects of local geology on ground motion near San Francisco Bay. Bull. Seismol. Soc. Am., 60, 29-61.

Borcherdt, R. D., 1994: Estimates of site-dependent response spectra for design (methodology and justification). Earthq. Spectra, 10, 617-653, doi: 10.1193/1.1585791. [Link]

Cadet, H., P. Y. Bard, and A. Rodriguez-Marek, 2010: Defining a standard rock site: Propositions based on the KiK-net database. Bull. Seismol. Soc. Am., 100, 172195, doi: 10.1785/0120090078. [Link]

Campbell, K. W., 1981: Near-source attenuation of peak horizontal acceleration. Bull. Seismol. Soc. Am., 71, 2039-2070.

Campbell, K. W., 2003: Strong-motion attenuation relations. In: Lee, W. H. K., H. Kanamori, P. C. Jennings, and C. Kisslinger (Eds.), International Handbook of Earthquake and Engineering Seismology, International Geophysics, Vol. 81, part B, Academic Press, 10031012, doi: 10.1016/S0074-6142(03)80174-2. [Link]

Campbell, K. W. and Y. Bozorgnia, 2007: Campbell-Bozorgnia NGA ground motion relations for the geometric mean horizontal component of peak and spectral ground motion parameters. Report PEER 2007/02, Pacific Earthquake Engineering Research Center.

Choi, Y. and J. P. Stewart, 2005: Nonlinear site amplification as function of $30 \mathrm{~m}$ shear wave velocity. Earthq. Spectra, 21, 1-30, doi: 10.1193/1.1856535. [Link]

Douglas, J., 2003: Earthquake ground motion estimation using strong-motion records: A review of equations for the estimation of peak ground acceleration and response spectral ordinates. Earth-Sci. Rev., 61, 43-104, doi: 10.1016/S0012-8252(02)00112-5. [Link]

Drouet, S., S. Chevrot, F. Cotton, and A. Souriau, 2008: Simultaneous inversion of source spectra, attenuation parameters, and site responses: Application to the data of the French accelerometric network. Bull. Seismol.Soc. Am., 98, 198-219, doi: 10.1785/0120060215. [Link]

Gallipoli, M. R. and M. Mucciarelli, 2009: Comparison of Site Classification from $V_{S 30}, V_{S 10}$, and HVSR in Italy. Bull. Seismol. Soc. Am., 99, 340-351, doi: 10.1785/0120080083. [Link]

Huang, M. W., J. H. Wang, K. F. Ma, C. Y. Wang, J. H. Hung, and K. L. Wen, 2007: Frequency-dependent site amplifications with $f \geq 0.01 \mathrm{~Hz}$ evaluated from velocity and density models in central Taiwan. Bull. Seismol. Soc. Am., 97, 624-637, doi: 10.1785/0120060139. [Link] 
Huang, M. W., J. H. Wang, H. H. Hsieh, and K. L. Wen, 2009: High frequency site amplification evaluated from Borehole data in the Taipei Basin. J. Seismol., 13, 601-611, doi: 10.1007/s10950-009-9153-3. [Link]

Joyner, W. B. and D. M. Boore, 1993: Methods for regression analysis of strong-motion data. Bull. Seismol. Soc. Am., 83, 469-487.

Kuo, C. H., K. L. Wen, H. H. Hsieh, T. M. Chang, C. M. Lin, and C. T. Chen, 2011: Evaluating empirical regression equations for $V s$ and estimating $V s 30$ in northeastern Taiwan. Soil Dyn. Earthq. Eng., 31, 431-439, doi: 10.1016/j.soildyn.2010.09.012. [Link]

Kuo, C. H., K. L. Wen, H. H. Hsieh, C. M. Lin, T. M. Chang, and K. W. Kuo, 2012: Site Classification and Vs30 estimation of free-field TSMIP stations using the logging data of EGDT. Eng. Geol., 129-130, 68-75, doi: 10.1016/j.enggeo.2012.01.013. [Link]

Lee, C. T. and B. R. Tsai, 2008: Mapping Vs30 in Taiwan. Terr. Atmos. Ocean. Sci., 19, 671-682, doi: 10.3319/ TAO.2008.19.6.671(PT). [Link]

Lee, C. T., C. T. Cheng, C. W. Liao, and Y. B. Tsai, 2001: Site classification of Taiwan free-field strong-motion stations. Bull. Seismol. Soc. Am., 91, 1283-1297, doi: 10.1785/0120000736. [Link]

Lee, C. T., B. S. Hsieh, C. H. Sung, and P. S. Lin, 2012: Regional Arias intensity attenuation relationship for Taiwan considering $V_{S 30}$. Bull. Seismol. Soc. Am., 102, 129-142, doi: 10.1785/0120100268. [Link]

Liu, K. S. and Y. B. Tsai, 2005: Attenuation relationships of peak ground acceleration and velocity for crustal earthquakes in Taiwan. Bull. Seismol. Soc. Am., 95, 10451058, doi: 10.1785/0120040162. [Link]

Liu, K. S., T. C. Shin, and Y. B. Tsai, 1999: A free-field strong motion network in Taiwan: TSMIP. Terr. At- mos. Ocean. Sci., 10, 377-396.

Liu, K. S., Y. B. Tsai, and P. S. Lin, 2013: A study on faulttype and site-effect $\left(V_{S 30}\right)$ parameters in the attenuation relationships of peak ground acceleration and peak ground velocity in Ilan, Taiwan.Bull. Seismol.Soc.Am., 103, 1823-1845, doi: 10.1785/0120120065. [Link]

National Earthquake Hazards Reduction Program (NEHRP), 1994: NEHRP Recommended Provisions for Seismic Regulations for New Buildings, Part 1 - Provisions, EEMA-222A, Federal Emergency Management Agency (FEMA), Building Seismic Safety Council, Washington, D.C., 335 pp.

National Earthquake Hazards Reduction Program (NEHRP), 1997: NEHRP Recommended Provisions for Seismic Regulations for New Buildings and Other Structures, Part 1: Provisions, FEMA 302, Federal Emergency Management Agency (FEMA), Building Seismic Safety Council, Washington, D.C., 336 pp.

National Earthquake Hazards Reduction Program (NEHRP), 2001: Recommended Provisions for Seismic Regulations for New Buildings and Other Structures, Part 1 - Provisions and Part 2 - Commentary, Report Numbers FEMA-368 and FEMA-369, Building Seismic Safety Council for the Federal Emergency Management Agency, Washington, D.C.

Power, M., B. Chiou, N. Abrahamson, Y. Bozorgnia, T. Shantz, and C. Roblee, 2008: An overview of the NGA project. Earthq. Spectra, 24, 3-21, doi: 10.1193/1.2894833. [Link]

Pratt, T. L., T. M. Brocher, C. S. Weaver, K. C. Creager, C. M. Snelson, R. S. Crosson, K. C. Miller, and A. M. Tréhu, 2003: Amplification of seismic waves by the Seattle basin, Washington State. Bull. Seismol. Soc. Am., 93, 533-545, doi: 10.1785/0120010292. [Link] 